\title{
Progress in Constraining Nuclear Symmetry Energy Using Neutron Star Observables Since GW170817
}

\author{
Bao-An Li ${ }^{1, *(D)}$, Bao-Jun Cai ${ }^{2}$, Wen-Jie $X^{3}{ }^{3}$ and Nai-Bo Zhang ${ }^{4}$ \\ 1 Department of Physics and Astronomy, Texas A\&M University-Commerce, Commerce, TX 75429-3011, USA \\ 2 Quantum Machine Learning Laboratory, Shadow Creator Inc., Shanghai 201208, China; \\ baojun.cai@ivglass.com \\ 3 Department of Physics, Yuncheng University, Yuncheng 044000, China; xiewenjie@ycu.edu.cn \\ 4 School of Physics, Southeast University, Nanjing 211189, China; naibozhang@seu.edu.cn \\ * Correspondence: Bao-An.Li@TAMUC.edu
}

Citation: Li, B.-A.; Cai, B.-J.; Xie, W.-J.; Zhang, N.-B. Progress in Constraining Nuclear Symmetry Energy Using Neutron Star Observables Since GW170817. Universe 2021, 7, 182. https:// doi.org/10.3390/universe7060182

Academic Editors: Ignazio Bombaci and Rosa Poggiani

Received: 10 May 2021

Accepted: 31 May 2021

Published: 4 June 2021

Publisher's Note: MDPI stays neutral with regard to jurisdictional claims in published maps and institutional affiliations.

Copyright: (C) 2021 by the authors Licensee MDPI, Basel, Switzerland. This article is an open access article distributed under the terms and conditions of the Creative Commons Attribution (CC BY) license (https:// creativecommons.org/licenses/by/ $4.0 /)$.
Abstract: The density dependence of nuclear symmetry energy is among the most uncertain parts of the Equation of State (EOS) of dense neutron-rich nuclear matter. It is currently poorly known especially at suprasaturation densities partially because of our poor knowledge about isovector nuclear interactions at short distances. Because of its broad impacts on many interesting issues, pinning down the density dependence of nuclear symmetry energy has been a longstanding and shared goal of both astrophysics and nuclear physics. New observational data of neutron stars including their masses, radii, and tidal deformations since GW170817 have helped improve our knowledge about nuclear symmetry energy, especially at high densities. Based on various model analyses of these new data by many people in the nuclear astrophysics community, while our brief review might be incomplete and biased unintentionally, we learned in particular the following: (1) The slope parameter $L$ of nuclear symmetry energy at saturation density $\rho_{0}$ of nuclear matter from 24 new analyses of neutron star observables was about $L \approx 57.7 \pm 19 \mathrm{MeV}$ at a $68 \%$ confidence level, consistent with its fiducial value from surveys of over 50 earlier analyses of both terrestrial and astrophysical data within error bars. (2) The curvature $K_{\text {sym }}$ of nuclear symmetry energy at $\rho_{0}$ from 16 new analyses of neutron star observables was about $K_{\text {sym }} \approx-107 \pm 88 \mathrm{MeV}$ at a $68 \%$ confidence level, in very good agreement with the systematics of earlier analyses. (3) The magnitude of nuclear symmetry energy at $2 \rho_{0}$, i.e., $E_{\text {sym }}\left(2 \rho_{0}\right) \approx 51 \pm 13 \mathrm{MeV}$ at a $68 \%$ confidence level, was extracted from nine new analyses of neutron star observables, consistent with the results from earlier analyses of heavy-ion reactions and the latest predictions of the state-of-the-art nuclear many-body theories. (4) While the available data from canonical neutron stars did not provide tight constraints on nuclear symmetry energy at densities above about $2 \rho_{0}$, the lower radius boundary $R_{2.01}=12.2 \mathrm{~km}$ from NICER's very recent observation of PSR J0740+6620 of mass $2.08 \pm 0.07 M_{\odot}$ and radius $R=$ $12.2-16.3 \mathrm{~km}$ at a $68 \%$ confidence level set a tight lower limit for nuclear symmetry energy at densities above $2 \rho_{0}$. (5) Bayesian inferences of nuclear symmetry energy using models encapsulating a firstorder hadron-quark phase transition from observables of canonical neutron stars indicated that the phase transition shifted appreciably both $L$ and $K_{\text {sym }}$ to higher values, but with larger uncertainties compared to analyses assuming no such phase transition. (6) The high-density behavior of nuclear symmetry energy significantly affected the minimum frequency necessary to rotationally support GW190814's secondary component of mass (2.50-2.67) $M_{\odot}$ as the fastest and most massive pulsar discovered so far. Overall, thanks to the hard work of many people in the astrophysics and nuclear physics community, new data of neutron star observations since the discovery of GW170817 have significantly enriched our knowledge about the symmetry energy of dense neutron-rich nuclear matter.

Keywords: equation of state; symmetry energy; neutron stars; Bayesian analysis; quark-hadron phase transition; tidal deformability; GW170817; GW190814; PSR J0740+6620; PSR J0030+0451 


\section{Introduction}

Understanding the nature and constrain the Equation of State (EOS) of dense neutronrich nuclear matter is a major science goal [1-4] shared by many other astrophysical observations (see, e.g., the analyses and reviews in [5-21]) and terrestrial nuclear experiments (see, e.g., [22-33]). However, realizing this goal is very challenging for many scientific and technical reasons. The EOS of Asymmetric Nuclear Matter (ANM) at nucleon density $\rho=\rho_{n}+\rho_{p}$ and isospin asymmetry $\delta \equiv\left(\rho_{n}-\rho_{p}\right) / \rho$ can be expressed in terms of the nuclear pressure $P(\rho, \delta)=\rho^{2} \frac{d \epsilon(\rho, \delta) / \rho}{d \rho}$ as a function of nucleon energy density $\epsilon(\rho, \delta)=\rho E(\rho, \delta)+\rho M$ where $E(\rho, \delta)$ and $M$ are the average nucleon energy and mass, respectively. $E(\rho, \delta)$ can be well approximated by [34]:

$$
E(\rho, \delta)=E_{0}(\rho)+E_{\text {sym }}(\rho) \cdot \delta^{2}+\mathcal{O}\left(\delta^{4}\right)
$$

according to essentially all existing nuclear many-body theories. The first term $E_{0}(\rho) \equiv E_{\mathrm{SNM}}(\rho)$ is the nucleon energy in Symmetric Nuclear Matter (SNM), having equal numbers of neutrons and protons, while the symmetry energy $E_{\text {sym }}(\rho)$ quantifies the energy needed to make nuclear matter more neutron rich. Since the pressure in ANM can be written as:

$$
P(\rho, \delta)=\rho^{2} \frac{d E(\rho, \delta)}{d \rho}=\rho^{2}\left[\frac{d E_{\mathrm{SNM}}(\rho)}{d \rho}+\frac{d E_{\mathrm{Sym}}(\rho)}{d \rho} \delta^{2}\right]=P_{\mathrm{SNM}}(\rho)+\rho^{2} \frac{d E_{\mathrm{Sym}}(\rho)}{d \rho} \delta^{2}
$$

where $P_{\mathrm{SNM}}(\rho) \equiv \rho^{2} \frac{d E_{\mathrm{SNM}}(\rho)}{d \rho}$ is the pressure in SNM, the pressure in Pure Neutron Matter (PNM) $P_{\mathrm{PNM}}(\rho) \equiv P(\rho, \delta=1)$ can be written as:

$$
P_{\mathrm{PNM}}(\rho)=P_{\mathrm{SNM}}(\rho)+\rho^{2} \frac{d E_{\mathrm{sym}}(\rho)}{d \rho} .
$$

By inverting the above equation, one can express the symmetry energy $E_{\mathrm{sym}}(\rho)$ as [35]:

$$
E_{\mathrm{sym}}(\rho)=E_{\mathrm{sym}}\left(\rho_{i}\right)+\int_{\rho_{i}}^{\rho} \frac{P_{\mathrm{PNM}}\left(\rho_{v}\right)-P_{\mathrm{SNM}}\left(\rho_{v}\right)}{\rho_{v}^{2}} d \rho_{v}
$$

where $\rho_{i}$ is a reference density. While many observables in terrestrial nuclear experiments have provided us much useful information about the pressure $P_{\mathrm{SNM}}$ in SNM over a broad density range [22], neutron star observables are messengers of nuclear pressure in neutronrich matter towards PNM. Combining all knowledge from analyzing the observables of both neutron stars and their mergers, as well as terrestrial nuclear experiments holds the promise of ultimately determining the density dependence of nuclear symmetry energy $E_{\text {sym }}(\rho)$.

The nuclear symmetry energy $E_{\text {sym }}(\rho)$ has broad ramifications for many properties of both isolated Neutron Stars (NSs) and gravitational waves from their mergers. For example, the density profile $\delta(\rho)$ of isospin asymmetry in NSs at $\beta$-equilibrium is uniquely determined by the $E_{\text {sym }}(\rho)$ through the $\beta$-equilibrium and charge neutrality conditions. Once the $\delta(\rho)$ is determined by the $E_{\text {sym }}(\rho)$, both the pressure $p(\rho, \delta)$ and energy density $\epsilon(\rho, \delta)$ reduce to functions of nucleon density only. Their relation $p(\epsilon)$ can then be used in solving the TOV equation to study NS structures and/or simulators of their mergers. It is well known that the critical nucleon density $\rho_{c}$ above which the fast cooling of protoneutron stars by neutrino emissions through the direct URCA process can occur, the crust-core transition density, and pressure in NSs all depend sensitively on $E_{\text {sym }}(\rho)$. Moreover, the frequencies and damping times of various oscillations, especially the $\mathrm{f}$ - and g-modes of the core, as well as the torsional mode of the crust, quadrupole deformations of isolated NSs, and the tidal deformability of NSs in inspiralling binaries all depend significantly on $E_{\text {sym }}(\rho)$.

Furthermore, the binding energy and spacetime curvature of NSs also depend on the symmetry energy $[36,37]$. In fact, there is a degeneracy between the EOS of super- 
dense neutron-rich matter and the strong-field gravity in determining the maximum mass of NSs. As was pointed out already [38], NSs are among the densest objects with the strongest gravity in the Universe, making them ideal places to test Einstein's General Relativity (GR) in regions where it has not been fully tested yet. One reason for testing GR in the extremely strong-gravity region is that there is no fundamental reason to choose Einstein's GR over other alternatives, and it is known that GR may break down at the limit of very strong gravitational fields. It was pointed out that the EOS-gravity degeneracy is tied to the fundamental Strong Equivalence Principle and can only be broken by using at least two independent observables [39]. It has also been shown that the variation of the mass-radius relation, especially the maximum NS mass an EOS can support, due to the variation of gravity theory (with respect to GR predictions), is much larger than that due to the uncertainty of the poorly known EOS of dense neutron-rich matter [40]. In fact, the EOS-gravity degeneracy has promoted some people to ask the question: Can the maximum mass of neutron stars rule out any EOS of dense stellar matter before the strong-field gravity is well understood? One possible answer is no [41]. One possible way out of the EOS-gravity degeneracy is to simultaneously determine both the strong-field gravity and the EOS of superdense matter using massive NSs. In this sense, the recent discovery of GW190814 with its secondary component in the NS-black hole mass gap is particularly interesting. Furthermore, the minimum frequency for GW190814's secondary component of mass $(2.50-2.67) M_{\odot}$ to be a supermassive and superfast pulsar that is $\mathrm{r}$-mode stable against run-away gravitational radiations depends critically on the high-density $E_{\text {sym }}(\rho)[42,43]$. Thus, a precise determination of $E_{\text {sym }}(\rho)$ has broad impacts in many areas of astrophysics, cosmology, and nuclear physics. In turn, many astrophysical observables from various compact objects and/or processes may carry useful information about nuclear symmetry energy. Indeed, various data of several observables, e.g., radii, masses, and tidal deformations of canonical neutron stars with masses around $1.4 M_{\odot}$, have been analyzed within different model frameworks to extract the symmetry energy and the EOS of SNM. Despite the vast diversity of approaches used, as we shall show, rather consistent results on the characteristics of symmetry energy around $\rho_{0}$ have been extracted, albeit within still relatively large error bars.

The symmetry energy $E_{\text {sym }}(\rho)$ at suprasaturation densities and the possible hadronquark phase transition are among the most uncertain parts of the EOS of dense neutronrich matter $[12,13,15,29]$. Moreover, the appearance of new particles, such as $\Delta(1232)$ resonances and various hyperons, also depends strongly on the high-density behavior of nuclear symmetry energy [44-59]. Since the nuclear symmetry energy will lose its physical meaning above the hadron-quark transition density, it is imperative to determine both the high-density $E_{\text {sym }}(\rho)$ and the properties of the hadron-quark phase transition simultaneously by using combined data from astrophysical observations and nuclear experiments. Since many existing studies assume that neutron stars are made of nucleons and leptons only and no hadron-quark phase transition is considered, it is thus interesting to compare the $E_{\text {sym }}(\rho)$ extracted from neutron stars with and without considering the hadron-quark phase transition.

Thanks to the great efforts of many people in both astrophysics and nuclear physics over the last two decades, significant progress has been made in constraining the symmetry energy $E_{\text {sym }}(\rho)$, especially around and below the saturation density of nuclear matter $\rho_{0}$. Compared to terrestrial experiments, neutron stars are particularly useful for probing the symmetry energy at suprasaturation densities. While still much less is known about $E_{\text {sym }}(\rho)$ at suprasaturation densities, astrophysical data since the detection of GW170817 have indeed stimulated many interesting new studies on nuclear symmetry energy in a broad density range. Results of these new studies together with those from earlier analyses of mostly terrestrial experiments have certainly improved our knowledge about the density dependence of nuclear symmetry energy. One of the main purposes of this brief review was to give an update on the systematics of the slope $L$ and curvature $K_{\text {sym }}$ of $E_{\text {sym }}(\rho)$ at $\rho_{0}$, as well as its magnitude at twice the saturation density, i.e., $E_{\mathrm{sym}}\left(2 \rho_{0}\right)$, based on 
recently published results from analyzing astrophysical data by the community since GW170817 was discovered. Moreover, as examples of how the nuclear symmetry energy affects astrophysical observables, we also briefly reviewed the following issues mostly based on our own recent work:

1. What have we learned about the symmetry energy from the tidal deformation of canonical neutron stars from GW170817, the mass of PSR J0740+6620, and NICER's simultaneous observation of mass and radius of PSR J0030+0451 and PSR J0740+6620?

2. How do the symmetry energy parameters extracted from recent observations of neutron stars compare with what we knew before the discovery of GW170817 that were mostly from terrestrial experiments?

3. What can we learn about the high-density symmetry energy from future, more precise radius measurement of massive neutron stars?

4. What are the effects of hadron-quark phase transition on extracting the symmetry energy from neutron star observables? How does the symmetry energy affect the fraction and size of quark cores in hybrid stars?

5. What are the effects of symmetry energy on the nature of GW190814's second component of mass (2.50-2.67) $M_{\odot}$ ?

6. If all the characteristics of nuclear symmetry energy at saturation density $\rho_{0}$, e.g., its slope $L$, curvature $K_{\mathrm{sym}}$, and skewness $J_{\mathrm{sym}}$, are precisely determined by the astrophysical observations and/or terrestrial experiments, how do we use them to predict the symmetry energy at suprasaturation densities, such as $2-3 \rho_{0}$ ? Nuclear symmetry energy $E_{\mathrm{sym}}(\rho)$ is normally expanded or simply parameterized as a function of $\chi=\left(\rho-\rho_{0}\right) / 3 \rho_{0}$ in the form of $E_{\text {sym }}(\rho) \approx S+L \chi+2^{-1} K_{\text {sym }} \chi^{2}+6^{-1} J_{\text {sym }} \chi^{3}+\cdots$. However, such kinds of expansions/parameterizations do not converge at suprasaturation densities where $\chi$ is not small enough, hindering an accurate determination of high-density $E_{\text {sym }}(\rho)$. Is there a better way that one can predict accurately the symmetry energy at high densities using $L, K_{\mathrm{sym}}$, and $J_{\text {sym }}$ ?

Answers to these questions are expected to be useful for further understanding the nature and EOS of density neutron-rich nuclear matter using high-precision data especially from more advanced X-ray observatories and gravitational wave detectors, as well as terrestrial experiments, especially those at high-energy rare isotope beam laboratories being built around the world.

2. What Have We Learned about the Symmetry Energy from the Tidal Deformation of Canonical Neutron Stars from GW170817 as Well as NICER's Simultaneous Observations of Mass and Radius of PSR J0030+0451 and PSR J0740+6620? How Do They Compare with What We Knew before GW170817?

The detection of GW170817 marked the beginning of a new era in astronomy in particular and physics in general. The nuclear astrophysics community has studied many aspects of isolated neutron stars and their mergers. Various interesting physics have been extracted from analyzing the GW170817 event as documented in the literature. Here, we briefly summarize what we have learned about the symmetry energy from studying the tidal deformation of canonical neutron stars observed by LIGO/VIRGO together with the recent mass and radius measurements from other observatories. While our brief review here is probably not complete, we tried to be unbiased and hope to provide a useful picture for the community on this particular issue. 
2.1. Updated Systematics of Symmetry Energy Parameters at $\rho_{0}$ after Incorporating the Results of Recent Analyses of Neutron Star Observables since GW170817

Before reaching the hadron-quark phase transition density, the SNM EOS $E_{0}(\rho)$ and symmetry energy $E_{\text {sym }}(\rho)$ can be parameterized as:

$$
\begin{aligned}
E_{0}(\rho) & =E_{0}\left(\rho_{0}\right)+\frac{K_{0}}{2}\left(\frac{\rho-\rho_{0}}{3 \rho_{0}}\right)^{2}+\frac{J_{0}}{6}\left(\frac{\rho-\rho_{0}}{3 \rho_{0}}\right)^{3}, \\
E_{\text {sym }}(\rho) & =E_{\text {sym }}\left(\rho_{0}\right)+L\left(\frac{\rho-\rho_{0}}{3 \rho_{0}}\right)+\frac{K_{\text {sym }}}{2}\left(\frac{\rho-\rho_{0}}{3 \rho_{0}}\right)^{2}+\frac{J_{\text {sym }}}{6}\left(\frac{\rho-\rho_{0}}{3 \rho_{0}}\right)^{3}
\end{aligned}
$$

in solving the inverse-structure problems of neutron stars, such as Bayesian analysis. In this case, the posterior Probability Distribution Functions (PDFs) of the EOS parameters and their correlations are inferred directly from observational data. Conventionally, the $E_{0}(\rho)$ and $E_{\text {sym }}(\rho)$ predicted by nuclear many-body theories can be Taylor expanded around $\rho_{0}$ in the same form as above. In this case, the coefficients in the Taylor expansions are obtained from the predicted energy density functionals. The symmetry energy is then characterized by its magnitude $S \equiv E_{\text {sym }}\left(\rho_{0}\right)$, slope $L=\left[3 \rho \mathrm{d} E_{\text {sym }} / \mathrm{d} \rho\right]_{\rho_{0}}$, curvature $K_{\text {sym }}=\left[9 \rho^{2} \mathrm{~d}^{2} E_{\text {sym }} / \mathrm{d} \rho^{2}\right] \rho_{0}$, and skewness $J_{\text {sym }}=\left[27 \rho^{3} \mathrm{~d}^{3} E_{\text {sym }} / \mathrm{d} \rho^{3}\right] \rho_{0}$ at saturation density. Similarly, the SNM EOS is characterized by the binding energy $E_{0}\left(\rho_{0}\right)$, incompressibility $K_{0}$, and skewness $J_{0}$ at $\rho_{0}$. It is worth noting that while the parameterizations can have as many high-order terms as one wishes as long as one can find enough data to fix them, the Taylor expansions have the general issue of convergence at high densities, as discussed in detail recently in [60]. The above parameterizations are often used in metamodeling of neutron star EOSs in Bayesian inferences. On the other hand, even when the exact theoretical energy density functionals are used in either the inversion processes or directly comparing forward model predictions with observational data (mostly in the latter case), often, only the first few coefficients, e.g., $E_{\mathrm{sym}}\left(\rho_{0}\right), L$, and $K_{\mathrm{sym}}$, are reported and compared with those from other analyses. This practice is normally appropriate as the parameterizations themselves can be considered as energy density functionals. However, one should be cautious that simply plugging the extracted coefficients from comparing model predictions with data into the above parameterizations may not reproduce the underlying symmetry energy at high densities due to the convergence issue [60].

Before the discovery of GW170817, much effort was devoted to extracting $E_{\mathrm{sym}}\left(\rho_{0}\right)$ and $L$, as well as their correlation using mostly data from terrestrial experiments. For example, a survey of 29 analyses performed before 2013 found the fiducial of $E_{\text {sym }}\left(\rho_{0}\right)=31.6 \pm 2.7 \mathrm{MeV}$ and $L=58.9 \pm 16 \mathrm{MeV}$ [61]. These values were changed to $E_{\mathrm{sym}}\left(\rho_{0}\right)=31.7 \pm 3.2 \mathrm{MeV}$ and $L=58.7 \pm 28.1 \mathrm{MeV}$ in the 2016 survey of 53 analyses [9]. Interestingly, as more diverse approaches were used in analyzing some of the same data, the uncertainty ranges increased somewhat, while the mean values remained the same. During the same time, microscopic and ab initio theories made more accurate predictions. For instance, using a novel Bayesian approach to quantify the truncation errors in chiral Effective Field Theory (EFT) predictions for pure neutron matter and manybody perturbation theory with consistent nucleon-nucleon and three-nucleon interactions up to fourth-order in the EFT expansion, $E_{\text {sym }}\left(\rho_{0}\right)$ and $L$ were recently predicted to be $E_{\mathrm{sym}}\left(\rho_{0}\right)=31.7 \pm 1.1 \mathrm{MeV}$ and $L=59.8 \pm 4.1 \mathrm{MeV}$ [62], respectively. The mean values of these predictions were in excellent agreement with the fiducial values found earlier.

The discovery of GW170817 has triggered many analyses of neutron star observables, mostly the tidal deformability and radii, using various models. Most of these new analyses have actually used the existing fiducial values of $E_{\mathrm{sym}}\left(\rho_{0}\right)$ and $\mathrm{L}$ in setting their prior ranges, albeit using various prior PDFs. To the best of our knowledge, the resulting posterior means and $68 \%$ confidence intervals of $E_{\text {sym }}\left(\rho_{0}\right)$ are not much different from the fiducial values given above as most of the neutron star observable studies are not really sensitive to the variation of $E_{\text {sym }}\left(\rho_{0}\right)$. However, new values of $L$ and $K_{\text {sym }}$ have been extracted in many studies. 
Shown in Figure 1 are $L$ values from 24 new analyses of neutron star observables in comparison with those from the 2013 and 2016 surveys. The results collected here are likely incomplete, and purely theoretical predictions are not included unless they are explicitly compared with new data of neutron star observables since GW170817. The results displayed randomly from the left are from (1) Mondal et al. [63], (2) and (3) Malik et al. [64], (4), (5), and (6) Biswas et al. [65], (7) Tsang et al. [66], (8) Xie and Li [67], (9) Baillot d'Etivaux et al. [68], (10) Malik et al. [69], (11) Zhao and Lattimer [70], (12) Lim and Holt [71], (13) Margueron et al. [72], (14) Drischler et al. [62], (15) Tan et al. [73], (16) Zhang et al. [74], (17) Chamel et al. [75], (18) Huang et al. [76], (19) Tews et al. [77], (20) Gil et al. [78], (21) Raithel and Özel [79], (22) Yue et al. [80], (23) Essick et al. [81], and (24) Li et al. [21].

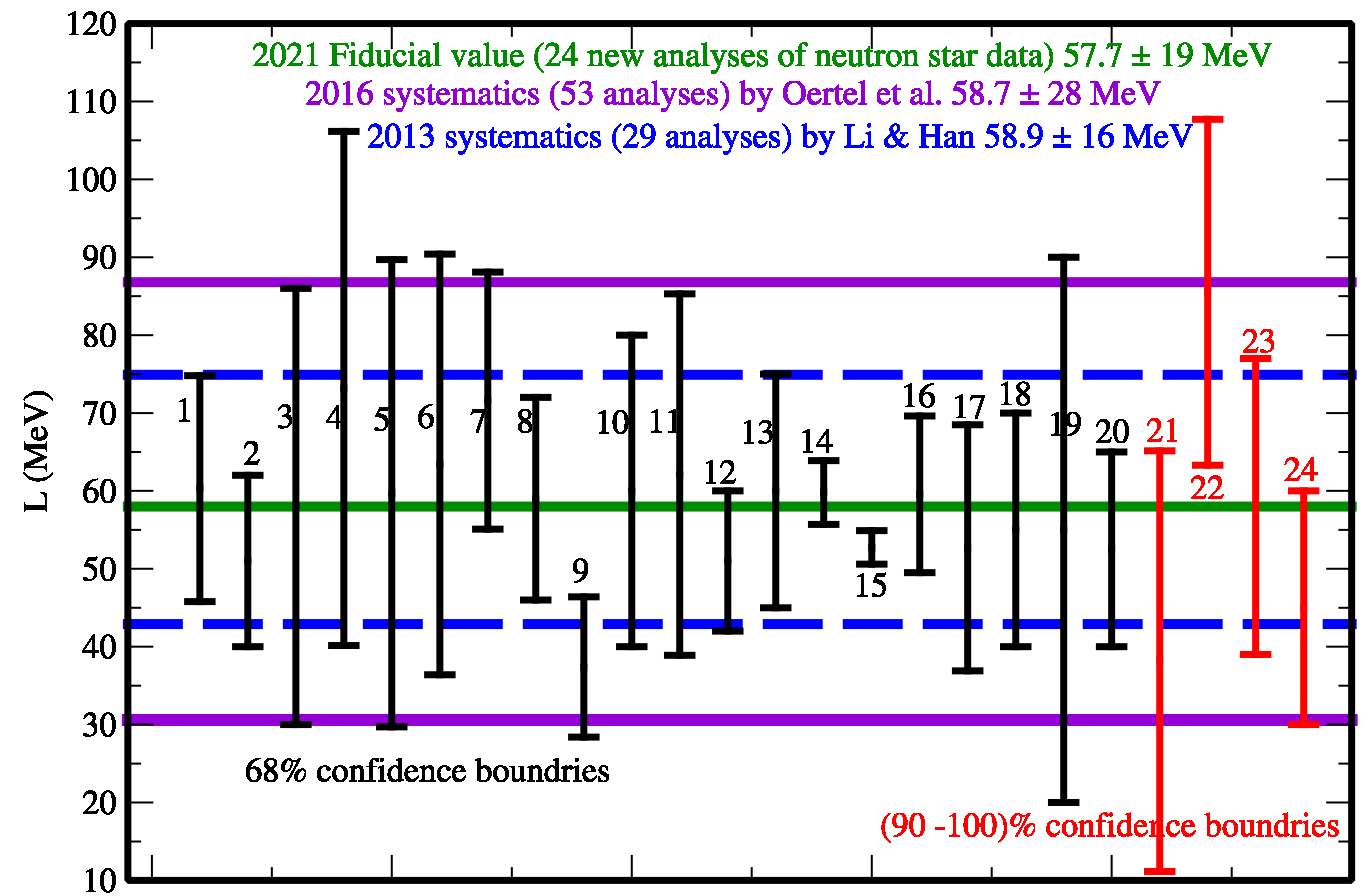

Figure 1. The slope parameter $L$ of nuclear symmetry from the (1) 2013 survey of 29 analyses of terrestrial experiments and astrophysical observations (between the dashed blue lines) [61], (2) 2016 surveys of 53 analyses (between the violet lines) [9], and (3) 24 new analyses of neutron star observables since GW170817 (see the detailed list in the text). The green line is the average value of $L$ from these 24 new analyses.

Interestingly, they are rather consistent with each other within the error bars. While it is beyond our ability to discuss the detailed differences among these analyses using vastly different models, obviously, reducing the error bars is one of the future tasks. A major contribution to the error bars is the correlation between $L$ and the even less constrained $K_{\text {sym }}$ parameter [82]. The average value of $L$ from these 24 new analyses of neutron star observables was about $L \approx 57.7 \pm 19 \mathrm{MeV}$ at a $68 \%$ confidence level, as indicated by the green line. Not so surprisingly, this was quite consistent with the fiducial values from both the 2013 and 2016 surveys within the error bars. If one considers all results equally reliable within the published error bars, the fiducial value of $L$ remains about $58 \mathrm{MeV}$ from, now in total, about 80 independent analyses of various observables of NSs and nuclear experiments, while there is not much reduction of its error bar. In fact, we noticed that the estimation of the error bars for the fiducial value of $L$ was not scientifically very rigorous as the nature, approach, and data used in the vastly different analyses were not completely transparent and compatible.

While the focus of this review was on the progress in constraining nuclear symmetry energy using NS observables since GW170817, it is worth noting that continuous efforts 
have been made in nuclear physics to constrain the symmetry energy. Indeed, there are many interesting results in the literature. In particular, we noticed that the fiducial value of $L \approx 57.7 \pm 19 \mathrm{MeV}$ at a $68 \%$ confidence level from the 24 new analyses of NS observables since GW170817, as well as the $L=58.9 \pm 16 \mathrm{MeV}$ from the 2013 survey of 29 analyses and the $L=58.7 \pm 28.1 \mathrm{MeV}$ from the 2016 survey of 53 analyses were all consistent with the latest report of $L$ between 42 and $117 \mathrm{MeV}$ from studying the pion spectrum ratio in heavy-ion collision in an experiment performed at RIKEN [83], but in serious tension with the implications of both the PREX-I and PREX-II experiments measuring the size of neutron skin in ${ }^{208} \mathrm{~Pb}$ using parity violating electron scatterings. The PREX-II experiment found very recently a neutron skin in ${ }^{208} \mathrm{~Pb}$ of size $R_{n}-R_{p}=0.283 \pm 0.071 \mathrm{fm}$. This implies a value of $L=106 \pm 37 \mathrm{MeV}$ [84] based on the Relativistic Mean Field (RMF) model calculations [85].

On the other hand, to be consistent with the results from other nuclear experiments including the sizes of neutron skins in several Sn isotopes, neutron star observables, as well as the state-of-the-art chiral EFT prediction, the neutron skin in ${ }^{208} \mathrm{~Pb}$ was predicted to be 0.17-0.18 fm based on Bayesian analyses using mocked data before the PREX-II result was announced [86]. Using a similar approach with essentially the same data sets of NS observables and neutron skin in Sn isotopes, a more recent analysis [81] found $L=58 \pm 19 \mathrm{MeV}$ and a neutron skin of $0.19_{-0.04}^{+0.03} \mathrm{fm}$ for ${ }^{208} \mathrm{~Pb}$, in good agreement with that found in [86] and the systematics discussed above. These interesting agreements and disagreements require further studies by the community. While finishing up this review, we learned about the very recent work of Biswas, who just performed a comprehensive Bayesian analysis using the latest NS observational data available (GW170817, NICER, and the revised mass measurement of PSR J0740+6620) and the PREX-II result [87]. Before adding the PREX-II results, the bound on $L$ was $61_{-16}^{+17} \mathrm{MeV}$ at a $1 \sigma$ confidence level, consistent with the systematics discussed above. After including the PREX-II data, $L$ becomes $69_{-16}^{+16} \mathrm{MeV}$. This is not much different from the result obtained from using only the astrophysics data, which dominate the whole data set used and have relatively smaller errors $(4 \sim 7 \%)$ for the NS radii compared to the uncertainty $(\sim 25 \%)$ for the neutron skin for ${ }^{208} \mathrm{~Pb}$ from PREX-II. Moreover, the inferred posterior value of neutron skin $R_{\text {skin }}^{208}=0.20_{-0.04}^{+0.04} \mathrm{fm}$ is significantly smaller than the PREX-II measured value, but consistent with that found in $[81,86]$ using similar approaches. Furthermore, the curvature of symmetry energy $K_{\text {sym }}$ inferred is $K_{\text {sym }}=-163_{-107}^{+123} \mathrm{MeV}$, consistent with its fiducial value, which we shall discuss next.

Shown in Figure 2 is a comparison of $K_{\text {sym }}$ from 16 new analyses of neutron star observables since GW170817 with respect to $(1) K_{\text {sym }} \approx-112 \pm 71 \mathrm{MeV}$ from the 2017 systematics by Mondal et al. [63] from analyzing the predictions of over 500 energy density functionals under the constraints of both terrestrial experiments and astrophysical observations available at the time and $(2) K_{\text {sym }} \approx-100 \pm 100 \mathrm{MeV}$ from the 2018 systematics by Margueron et al. [72] from a metamodeling of nuclear EOS under similar constraints, respectively. Most of the 16 analyses are the same ones used in Figure 1, but not all analyses gave simultaneously both the $L$ and $K_{\text {sym }}$ values by marginalizing one of them. More specifically, (1) and (2) were from Malik et al. [64] and (3), (4), and (5) from Biswas et al. [65]. The rest were from (6) Zimmerman et al. [88], (7) Tsang et al. [66], (8) Xie and Li [67], (9) Baillot d'Etivaux et al. [68], (10) Malik et al. [69], (11) Lim and Holt [71], (12) Chamel et al. [75], (13) Raithel and Özel [79], (14) Carson et al. [89], (15) Yue et al. [80], and (16) Essick et al. [81]. The average of these 16 new analyses was about $K_{\text {sym }} \approx-107 \pm 88 \mathrm{MeV}$ at a $68 \%$ confidence level. Obviously, this was in very good agreement with both the 2017 and 2018 systematics shown in the same plot.

It is understood that, within the errors, $L$ and $K_{\text {sym }}$ are generally correlated. Their correlations have significant effects on a number of neutron star properties [11]. While the individual values of $L$ and $K_{\text {sym }}$ shown in Figures 1 and 2 are useful, future constraints on their correlations will also be important [82,90-92]. 


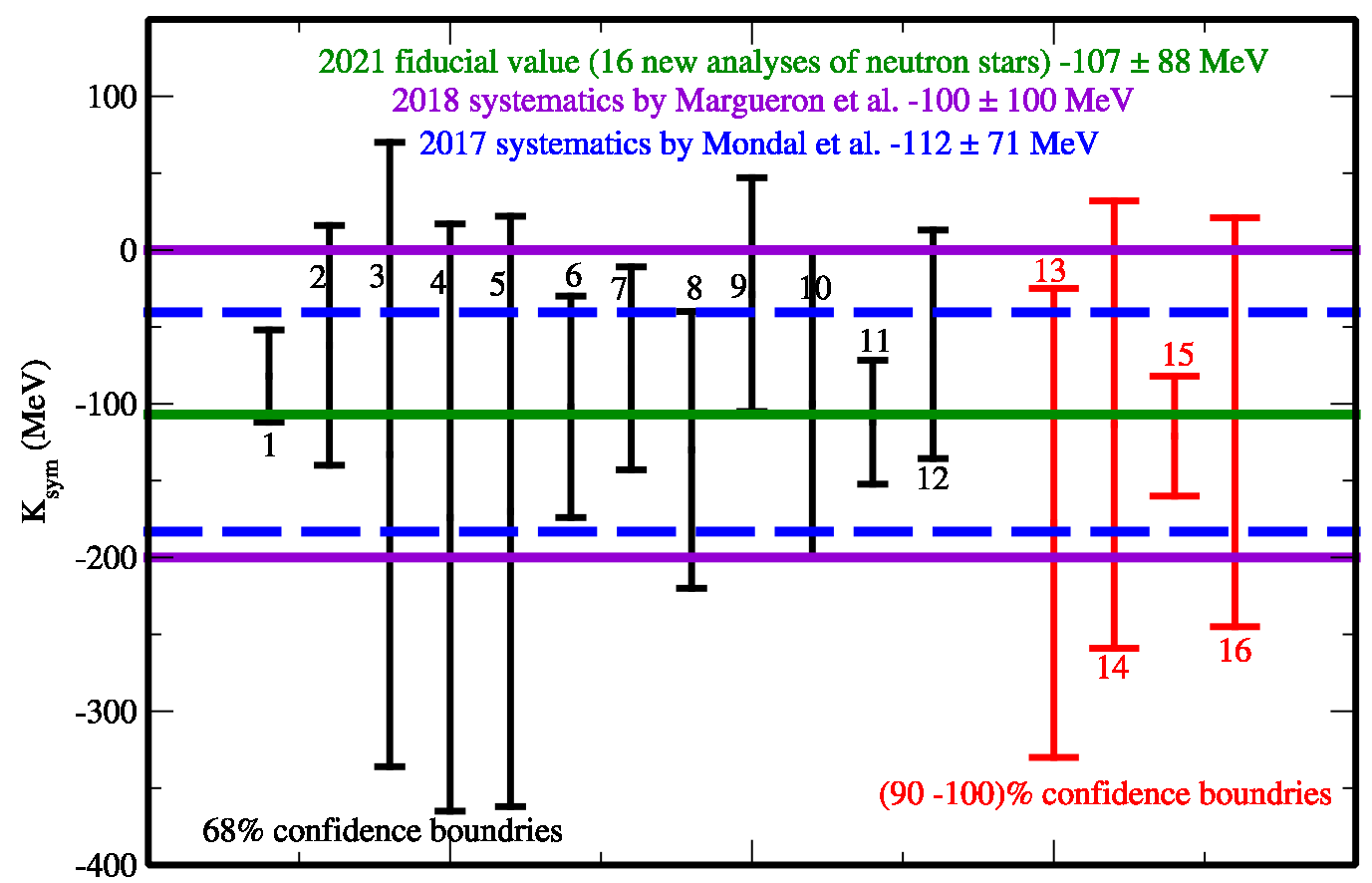

Figure 2. The curvature parameter $K_{\text {sym }}$ of nuclear symmetry from the (1) 2017 survey of 500 energy density functional predictions constrained by available terrestrial experiments and astrophysical observations (between the dashed blue lines) [63], (2) 2018 surveys based on a metamodel of nuclear EOS under similar constraints (between the violet lines) [72], and (3) 16 new analyses of neutron star observables since GW170817 (see the detailed list in the text). The green line is the average value of $K_{\text {sym }}$ from these 16 new analyses.

\subsection{Symmetry Energy at $2 \rho_{0}$ Extracted from Neutron Star Observables}

Besides constraining the characteristics of the symmetry energy at $\rho_{0}$ discussed above, recent neutron star observations have also been used to constrain explicitly the symmetry energy at suprasaturation densities. As an example, shown in Figure 3 is the magnitude of symmetry energy at twice the saturation density of nuclear matter from nine recent analyses of neutron stars in comparison with the two results from earlier heavy-ion reaction experiments (from the FOPI-LAND [93] and the ASY-EOS [94] Collaborations by analyzing the relative flows and yields of light mirror nuclei, as well as neutrons and protons in heavy-ion collisions at beam energies of $400 \mathrm{MeV} /$ nucleon). More specifically, the nine analyses were from (1) (Zhang and Li 2019) directly inverting observed NS radii, tidal deformability, and maximum mass in the high-density EOS space [95-97], (2) (Xie and Li 2019) a Bayesian inference from the radii of canonical NSs observed by using Chandra X-rays and gravitational waves from GW170817 [98], (3) (Zhou et al., 2019) analyses of NS radii, tidal deformability, and maximum mass within an extended Skyrme-HartreeFock approach (eSHF) [99], (4) (Nakazato and Suzuki 2019) analyzing cooling timescales of protoneutron stars, as well as the radius and tidal deformability of GW170817 [100], (5) (d'Etivaux et al., 2019) a Bayesian inference directly from the X-ray data of seven quiescent low-mass X-ray binaries in globular clusters [68], (6) (Xie and Li 2020) a Bayesian inference from the radii of NSs observed by NICER and LIGO/VIRGO [67], (7) (Tsang et al., 2020) Bayesian analyses of tidal deformation of canonical NSs from LIGO/VIRGO [66], (8) (Yue et al., 2021) eSHF analyses of tidal deformation from GW170817 and radii from NICER [80], and (9) (Zhang et al., 2021) Skyrme-Hartree-Fock predictions with interaction parameters constrained by heavy-ion reaction experiments, the neutron skin of heavy nuclei, as well as the tidal deformation and radii of neutron stars from LIGO/VIRGO [74]. 


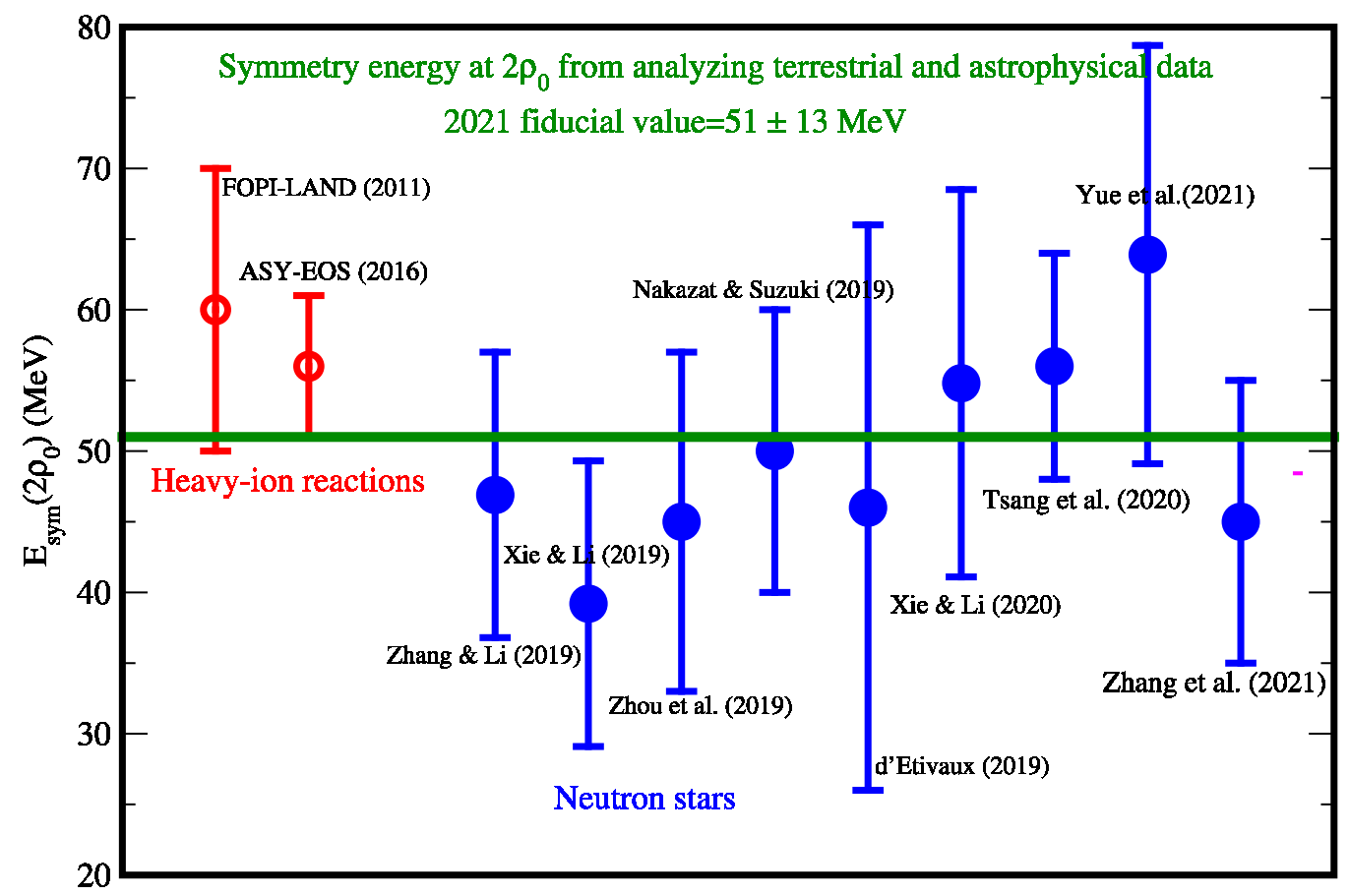

Figure 3. Nuclear symmetry $E_{\text {sym }}\left(2 \rho_{0}\right)$ at twice the saturation density of nuclear matter extracted from earlier heavy-ion reaction experiments in terrestrial nuclear laboratories (red) and 9 recent analyses of neutron star observables (see the text for the detailed list). The green line serving as the latest fiducial value of $E_{\text {sym }}\left(2 \rho_{0}\right)$ is the global average of all points shown.

While certainly the model dependence and the error bars are still relatively large, all results from both heavy-ion reactions and neutron stars scatter around an overall mean of $E_{\text {sym }}\left(2 \rho_{0}\right) \approx 51 \pm 13 \mathrm{MeV}$ at a $68 \%$ confidence level, as indicated by the green line. The symmetry energy around $2 \rho_{0}$ is particularly interesting because the pressure around this density determines the radii of canonical NSs [5]. Moreover, around $(1-2) \rho_{0}$, the symmetry energy contribution to the NS pressure competes strongly with that from SNM [25]. This is also the density range where the current chiral EFT and other ab initio theories are still applicable. It is thus interesting to note that the latest many-body perturbation theory calculations with consistent nucleon-nucleon and three-nucleon interactions up to fourth-order in the chiral EFT expansion predicted a value of $E_{\text {sym }}\left(2 \rho_{0}\right) \approx 45 \pm 3 \mathrm{MeV}$ [62]. Similarly, the latest quantum Monte Carlo calculations using local interactions derived from the chiral EFT up to next-to-next-to-leading order predicted a value of $E_{\text {sym }}\left(2 \rho_{0}\right) \approx 46 \pm 4$ $\mathrm{MeV}$ [101]. Obviously, the fiducial value of $E_{\text {sym }}\left(2 \rho_{0}\right)$ from the analyses listed above was in good agreement with these state-of-the-art nuclear theory predictions, albeit with a significantly larger error bar.

\subsection{Directly Solving Neutron Star Inverse-Structure Problems in the High-Density EOS Parameter Space}

How are the constraints on $E_{\text {sym }}\left(2 \rho_{0}\right)$ or generally on the density dependence of nuclear symmetry energy $E_{\text {sym }}(\rho)$ at suprasaturation densities extracted from neutron star observables? To answer this question, we provide two examples from our own recent work. The first example presented in the following is the direct inversion of several NS observables in a three-dimensional high-density EOS parameter space [42]. Another example to be presented in the next subsection is from Bayesian statistical analyses of the same NS observables.

Since the magnitude and slope of symmetry energy, as well as the binding energy and incompressibility of SNM at $\rho_{0}$ are relatively well determined, as we discussed above, they can be fixed at their currently known most probable values given above. One can then metamodel NS EOSs in the three-dimensional $J_{0}-K_{\text {sym }}-J_{\text {sym }}$ high-density EOS 
parameter space. Given an NS observable, one can find all points (EOSs) necessary to reproduce the observable, thus numerically solving the NS inverse-structure problem. Such an approach has been found very successful in several applications [95-97,102].

As an example, the left window of Figure 4 illustrates how neutron star radii and the tidal deformation $\Lambda_{1.4}$ of canonical NSs from Chandra, GW170817 [103], and NICER [104], as well as the presently observed NS maximum mass $M_{\max }$ and causality condition together can constrain the high-density EOS parameter space spanned in $J_{0}-K_{\text {sym }}-J_{\text {sym }}$ using $E_{\text {sym }}\left(\rho_{0}\right)=31.6 \mathrm{MeV}$ and $L=58.9 \mathrm{MeV}$. On each surface, the indicated NS observable is a constant, while on the causality surface, the central density of the maximum mass NS is equal to the critical density satisfying the causality condition (the speed of sound is equal to that of light) [96]. It is seen that while the skewness $J_{0}$ of SNM significantly affects the NS maximum mass (notice its change when the $M_{\max }$ changes from 2.01 to $2.14 M_{\odot}$ ), it has little effects on the radius or the tidal deformation, as indicated by the vertical surfaces of constant radii and tidal deformation. On the other hand, all of the observables shown and the causality surface depend sensitively on the high-density $E_{\text {sym }}(\rho)$ parameters $K_{\text {sym }}$ and $J_{\text {sym }}$. The crosslines between the constant surfaces set upper and lower limits for $E_{\text {sym }}(\rho)$ and $J_{0}$. For example, the crossline between the surface of radius $R_{1.4}=12.83 \mathrm{~km}$ for a $1.4 M_{\odot}$ NS (it is the $68 \%$ confidence upper boundary from an earlier analysis of Chandra data [105] and very close to the $\Lambda_{1.4}=580$ surface from LIGO/VIRGO) and the causality surface sets an upper boundary for $E_{\text {sym }}(\rho)$, while the crossline between the causality surface and the surface of the previously reported NS maximum mass of $2.14 M_{\odot}$ from PSR J0740+6620 [106] sets approximately the lower limit for $E_{\text {sym }}(\rho)$ (note that the mass of PSR J0740+6620 was reduced from the previously published $M=2.14 M_{\odot}$ to $2.08 \pm 0.07 M_{\odot}$ recently [107-109]; for this review, we still used some of our previous results obtained from using $M=2.14 M_{\odot}$ ). The projections of the above two crosslines onto the $J_{\text {sym }}-K_{\text {sym }}$ plane are shown in the right window of Figure 4 . It is seen that these projections stringently limit the range of $K_{\text {sym }}$ compared to its prior range, but do not narrow down the range of $J_{\text {sym }}$, thus limited constraints on the symmetry energy at densities above about $2 \rho_{0}$.
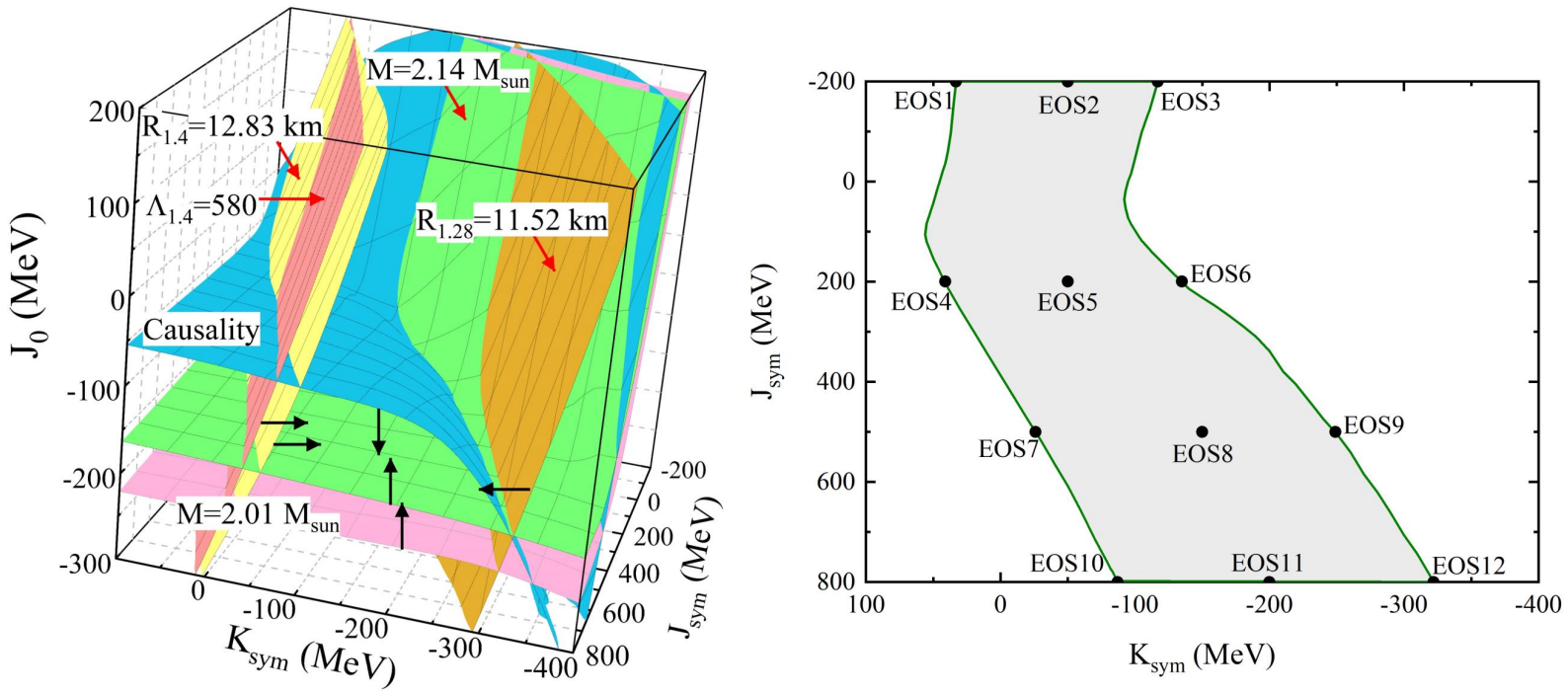

Figure 4. (Left) The constant surfaces of $\mathrm{M}=2.01 \mathrm{M}_{\odot}$ (pink surface), $\mathrm{M}=2.14 \mathrm{M}_{\odot}$ (green surface), $R_{1.4}=12.83 \mathrm{~km}$ (yellow surface), $\Lambda_{1.4}=580$ (red surface), $R_{1.28}=11.52 \mathrm{~km}$ (orange surface), and causality condition (blue surface) in the 3-dimensional parameter space of $K_{\text {sym }}-J_{\text {sym }}-J_{0}$. The black arrows show the directions supporting the corresponding constraints, and the red arrows direct to the corresponding surfaces. (Right) Boundaries of the symmetry energy in the $K_{\text {sym }}-J_{\text {sym }}$ plane. Modified from the figures originally published in [42,110].

Moreover, comparing the crossline between the causality surface and the surface of the NS maximum mass of $2.14 M_{\odot}$ (previously reported mass [106]) from PSR J0740+6620 with 
that between the causality surface and the previously known NS maximum mass of $2.01 \mathrm{M}_{\odot}$ from PSR J0348+0432, it is seen that the NS maximum mass used clearly affects the lower boundary of symmetry energy at suprasaturation densities. Detailed analyses $[97,111]$ indicated that the change of the observed NS maximum mass from 2.01 to $2.14 M_{\odot}$ mostly affects the $E_{\text {sym }}(\rho)$ above about $2 \rho_{0}$, and with $2.14 M_{\odot}$, the high-density symmetry energy becomes appreciably stiffer. It is also interesting to note that the $R_{1.28}=11.52 \mathrm{~km}$ surface for a 1.28 $M_{\odot}$ NS from NICER's observation of PSR J0030+0451 [104,112] is slightly outside the crossline between the causality surface and the surface of NS of a maximum mass of $2.14 M_{\odot}$. Thus, this lower limit of radius for the light NS does not really provide additional constraints on the symmetry energy.

Very interestingly, the NICER Collaboration has just announced their radius measurement for PSR J0740+6620, which is the currently observed most massive NS. To avoid confusion in the following discussions, we noticed again here that this NS was reported earlier to have a mass of $2.14_{-0.09}^{+0.10} M_{\odot}$ [106]. Some of the results from our earlier publications were obtained by using $2.14 M_{\odot}$ as the minimum maximum mass of NSs. Now, it has an updated mass of $2.08 \pm 0.07 M_{\odot}[107-109,113]$. Miller et al. reported a radius of $13.7_{-1.5}^{+2.6} \mathrm{~km}(68 \%)[108,113]$ for PSR J0740+6620. Consistently, an independent analysis of the NICER data by Riley et al. found a radius of $12.39_{-0.98}^{+1.30} \mathrm{~km}$ [109]. Our discussions here used the data from Miller et al. as we first learned the NICER results from Miller's seminar earlier [113]. Results from using the data reported by Riley et al. were approximately the same within the remaining uncertainties of the $L$ parameter [114].

While the upper radius limit from Miller et al. or Riley et al. did not provide additional constraints on the upper boundary of symmetry energy compared to the upper radius limit of $R_{1.4}=12.83 \mathrm{~km}$ from the earlier analysis of Chandra data [105] or the upper limit of $\Lambda_{1.4}=580$ from LIGO/VIRGO, the lower radius limit of $R_{2.01}=12.2 \mathrm{~km}$ for PSR J0740+6620 as indicated by the blue surface in Figure 5 provides an even more stringent limit on the lower boundary of high-density symmetry energy compared to that discussed above. Moreover, comparing the $R_{2.01}=12.2 \mathrm{~km}$ surface with the minimum NS maximum mass surface of $2.01 M_{\odot}$ shown in Figure 4, one sees clearly the power of measuring both the mass and radius simultaneously of heavy NSs for the purposes of limiting the highdensity EOS parameter space. There is a large gap in the direction of $J_{0}$ between the two surfaces in the region where both $K_{\mathrm{sym}}$ and $J_{\text {sym }}$ are small (correspondingly, the symmetry energy is very soft). More specifically, the left side of the $R_{2.01}=12.2 \mathrm{~km}$ surface is the allowed high-density EOS parameter space. Before this new radius observation, the lower boundary of $E_{\mathrm{sym}}(\rho)$ was determined by the crossline between the causality surface and the surface of the NS maximum mass of $2.14 M_{\odot}$ shown in Figure 4 . It was now determined by the crossline between the causality surface and the surface of $R_{2.01}=12.2 \mathrm{~km}$. It was at significantly higher $K_{\text {sym }}$ values, moving the lower limit of $E_{\text {sym }}(\rho)$ upward. Moreover, if only the mass of $2.08 \pm 0.07 M_{\odot}$ were measured for PSR J0740+6620, then the lower boundary of the high-density symmetry energy would be determined by the crossline between the causality surface and the constant surface of $2.01 M_{\odot}$. It is on the right of the crossline between the causality and $\mathrm{M}=2.14 M_{\odot}$ surfaces, providing an even looser lower boundary for the high-density symmetry energy.

To see more quantitatively the relative locations of these boundaries and their constraints on the high-density symmetry energy, shown in the left window of Figure 6 are projections of the indicated crosslines of constant surfaces of NS observables to the $K_{\text {sym }}-J_{\text {sym }}$ plane with $L$ set at $L=58.7 \mathrm{MeV}$. The right window shows the resulting constraints on the symmetry energy. For quantitative comparisons with the systematics discussed earlier, the new bounds on $E_{\text {sym }}\left(2 \rho_{0}\right)$ and $E_{\text {sym }}\left(3 \rho_{0}\right)$ were also extracted and labeled. Clearly, the radius measurement for PSR J0740+6620 had a significant effect on refining the constraint on the lower boundary of high-density symmetry energy. This has very interesting implications to several predicted effects associated with the super-soft (decreasing symmetry energy with increasing density), e.g., the formation of proton polarons, 
kaon condensation and isospin separation instability in the cores of NSs; see [114] for more detailed discussions.

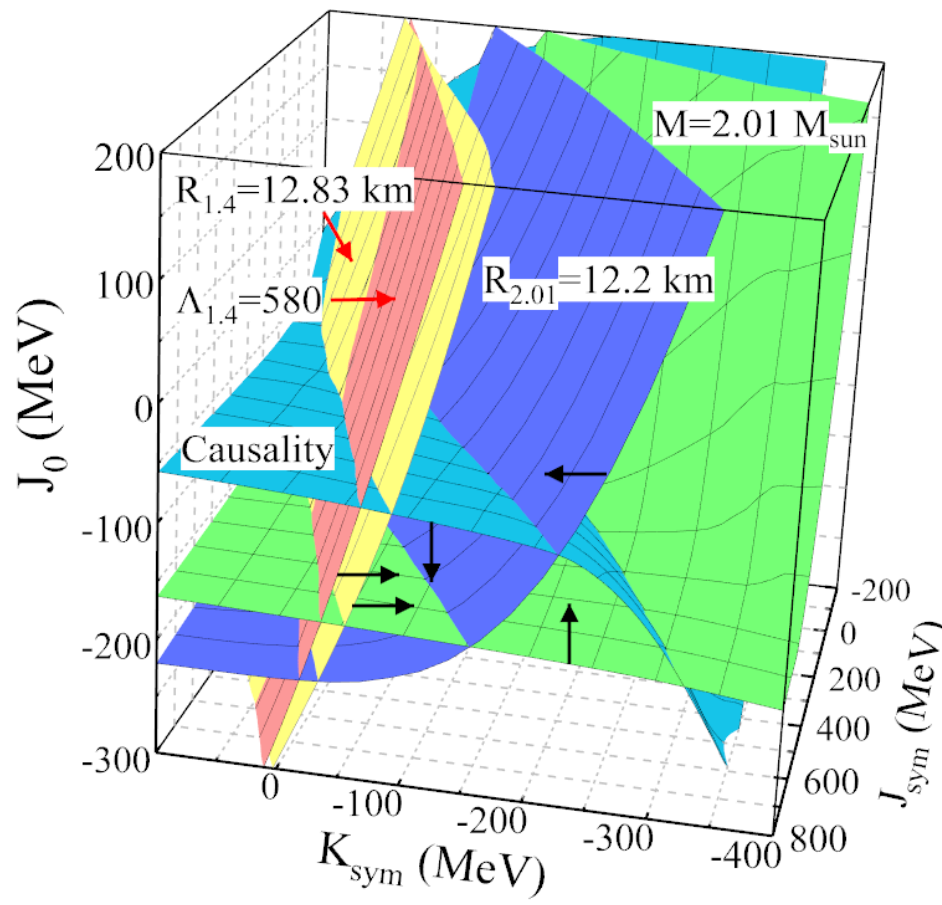

Figure 5. Similar to Figure 4, but with the constant surface of the minimum radius $R=12.2 \mathrm{~km}$ at a $68 \%$ confidence level for PSR J0740+6620 with a mass of $2.08 \pm 0.07 M_{\odot}$ [108].
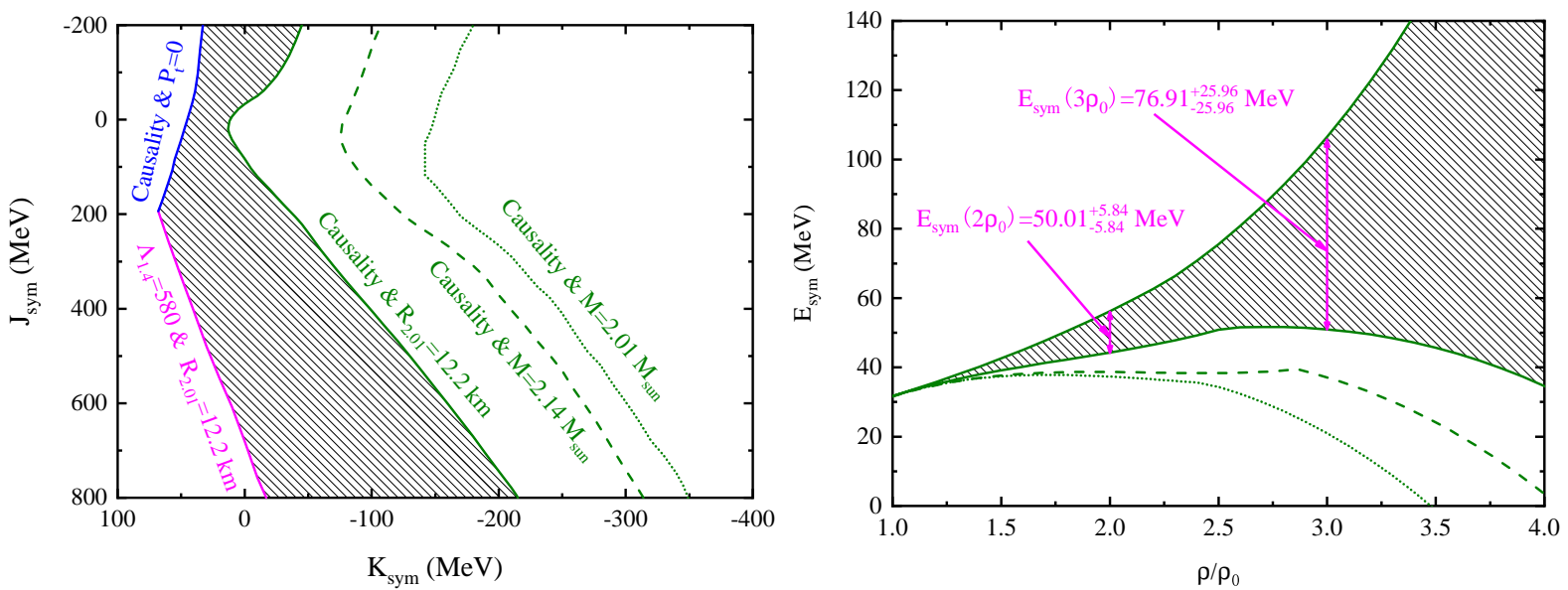

Figure 6. (Left) Projections of the indicated crosslines of constant surfaces of NS observables to the $K_{\text {sym }}-J_{\text {sym }}$ plane for $L=58.7 \mathrm{MeV}$. (Right) The constraints on symmetry energy extracted from the combinations of $K_{\text {sym }}-J_{\text {sym }}$ in the left plot. $E_{\text {sym }}\left(2 \rho_{0}\right)$ and $E_{\text {sym }}\left(3 \rho_{0}\right)$ are also extracted and labeled.

Furthermore, from the separations between the constant surfaces of $R_{1.4}=12.83 \mathrm{~km}$ and $R_{1.28}=11.52 \mathrm{~km}$ shown in the left window of Figure 4, as well as $R_{2.01}=12.2 \mathrm{~km}$ for PSR J0740+6620 shown in Figure 5, one can clearly see the importance of precise measurements of NS masses and radii for determining the symmetry energy. To see more clearly the effects of symmetry energy on the radius and tidal deformability, one can examine the constant surfaces of radius and tidal polarizability in the three-dimensional symmetry energy parameter space of $L-K_{\text {sym }}-J_{\text {sym }}$ by setting the skewness of SNM $J_{0}$ to a constant, as it has little effect on these two observables.

Shown in Figure 7 is an example for this purpose in the three-dimensional space within the known uncertainties of the three symmetry energy parameters by setting 
$J_{0}=-180 \mathrm{MeV}$. It is seen again that only one observable, either $R_{1.4}$ or $\Lambda_{1.4}$, is insufficient to completely determine the three parameters, but provides a strong constraint on their correlations. As mentioned earlier, since the average density reached in canonical NSs is not very high, except when $K_{\text {sym }}$ is very small (having large negative values), the high-density parameter $J_{\text {sym }}$ plays a small role in determining the radii or tidal deformations of these NSs, as indicated by their largely vertical surfaces. Interestingly, but not surprisingly, while $L$ dominates, $K_{\text {sym }}$ has an appreciable role in determining the radius and/or tidal deformation. This explains why the community has extracted both $L$ and $K_{\text {sym }}$ from analyzing GW170817, but not $J_{\text {sym }}$ yet, as we summarized in the previous section. It also indicates that it is insufficient to adjust $L$ in models or simply report $L$ without giving any information about the $K_{\text {sym }}$ parameter. For example, for $R_{1.4}=12 \mathrm{~km}$, it can be obtained with a large $L$, but small $K_{\text {sym }}$ or a small $L$, but larger $K_{\text {sym }}$. Therefore, a precise measurement of $R_{1.4}$ or $\Lambda_{1.4}$ alone is not sufficient to precisely fix $L$ and $K_{\text {sym }}$ individually. This is partially responsible for the large error bars of both of them shown in Figures 1 and 2 in the previous section. Moreover, the fact that $R_{1.4}$ and $\Lambda_{1.4}$ do not provide a stringent constraint on $J_{\text {sym }}$, as shown in Figure 7 (which is also verified by the Bayesian analysis to be discussed next), implies that these two observables do not constrain the symmetry energy at very high densities. We discuss this issue more quantitatively next.

The resulting boundaries of $E_{\mathrm{sym}}(\rho)$ from the crosslines of neutron star observables shown in Figure 4 and discussed above are shown as thick blue lines in Figure 8 in comparisons with the predictions of phenomenological models (left) and microscopic theories (right). Essentially, all existing nuclear many-body theories using all available nuclear forces have been used to predict the density dependence of nuclear symmetry energy $E_{\text {sym }}(\rho)$. Shown in the left window are sixty examples selected from six classes of over five-hundred twenty phenomenological models and/or energy density functional theories, while the right window shows eleven examples from microscopic and/or ab initio theories. Mostly by design, they all agree with existing constraints within error bars available around and below the saturation density $\rho_{0}$. However, at suprasaturation densities, their predictions diverge very broadly.
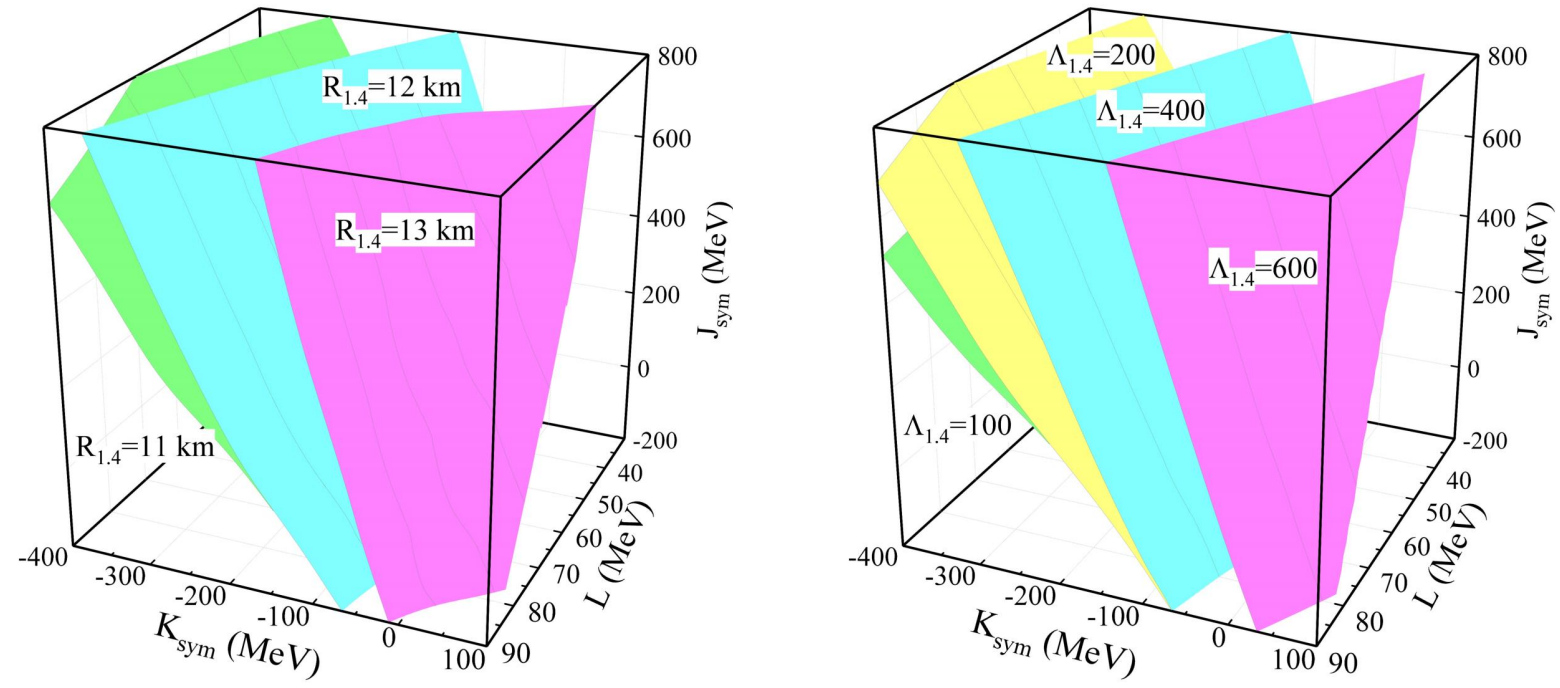

Figure 7. The constant surfaces of radius (left) and tidal polarizability (right) in the symmetry energy parameter space of $L-K_{\text {sym }}-J_{\text {sym }}$, respectively. Taken from [102].

The fundamental reason for the very uncertain high-density $E_{\text {sym }}(\rho)$ is our poor knowledge about the relatively weak isospin dependence (i.e., the difference between neutron-proton interactions in isotriplet and isosinglet channels and their differences from neutron-neutron and proton-proton interactions) of the two-body force, as well as the spin-isospin dependence of the three-body and tensor forces at short distances in dense 
neutron-rich nuclear matter. While the astrophysical constraints discussed above can rule out many model predictions up to about $2 \rho_{0}$, huge uncertainties remain at higher densities. This is mainly because the radii and/or tidal deformations of canonical NSs are mostly determined by the pressure around the average density $2 \rho_{0}$ in these NSs [5]. To constrain $E_{\text {sym }}(\rho)$ at higher densities, one thus has to use radii of more massive NSs, or messengers directly from the core of isolated NSs, e.g., neutrinos, or high-frequency gravitational waves from the post merger phase of colliding NSs. Moreover, many theories predict that at densities higher than about $(2 \sim 4) \rho_{0}$, a hadron-quark phase transition will occur. Since $E_{\text {sym }}(\rho)$ will lose its physical meaning once the hadron-quark phase transition happens, one thus has to extract the high-density $E_{\text {sym }}(\rho)$ from astrophysical observables using NS models that properly consider the hadron-quark phase transition.

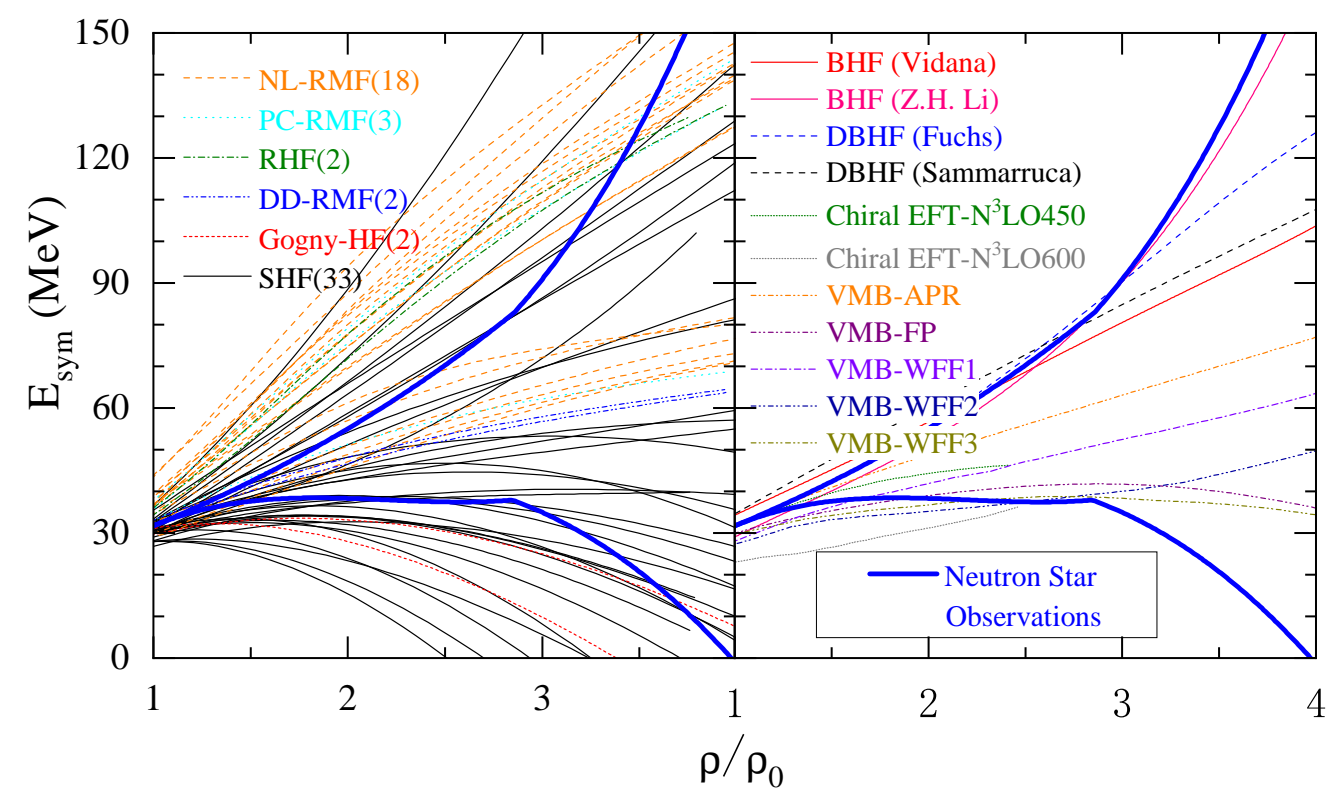

Figure 8. Examples of the density dependence of nuclear symmetry energy predicted by nuclear many-body theories using different interactions, energy density functionals, and/or techniques in comparison with the constraining boundaries extracted from studying the properties of neutron stars. Modified from the figures originally published in [42,110,115].

\subsection{Bayesian Inference of Symmetry Energy Parameters from the Radii of Canonical Neutron Stars}

While the direct inversion technique used in the examples given above has the advantage of enabling us to visualize how each observable may help constrain one or more high-density EOS parameters, it is limited to the three-dimensional space. It is well known that the general technique of multidimensional inversion is the Bayesian statistical inference. It has been widely used in analyzing various data within different EOS model frameworks. For example, albeit giving quantitatively slightly different results, several Bayesian analyses of the GW17817 data have indicated that the radii of canonical NSs are approximately mass independent $[14,103,116]$. For example, the principal NS in GW170817 has a mass between 1.36 and $1.58 M_{\odot}$, while its secondary has a mass between 1.18 and $1.36 M_{\odot}$ [103]. Assuming initially their radii are different in their model analyses, the LIGO/VIRGO Collaborations found a common radius $R_{1.4}=11.9 \pm 1.4 \mathrm{~km}$ for the two NSs involved. Using the later as the radius data (labeled as Reference in Figures 9-11), together with other general constraints, such as the minimum NS maximum mass of $1.97 \mathrm{M}_{\odot}$ and causality condition, in a Bayesian analysis using the metamodel of NS EOSs [67], the PDFs of the six EOS parameters were inferred and shown as the black curves in Figure 10. The other curves labeled as Case 1, 2, 3 are results of using mocked mass-radius relations shown in Figure 9. They will be discussed in detail in Section 2.5. 

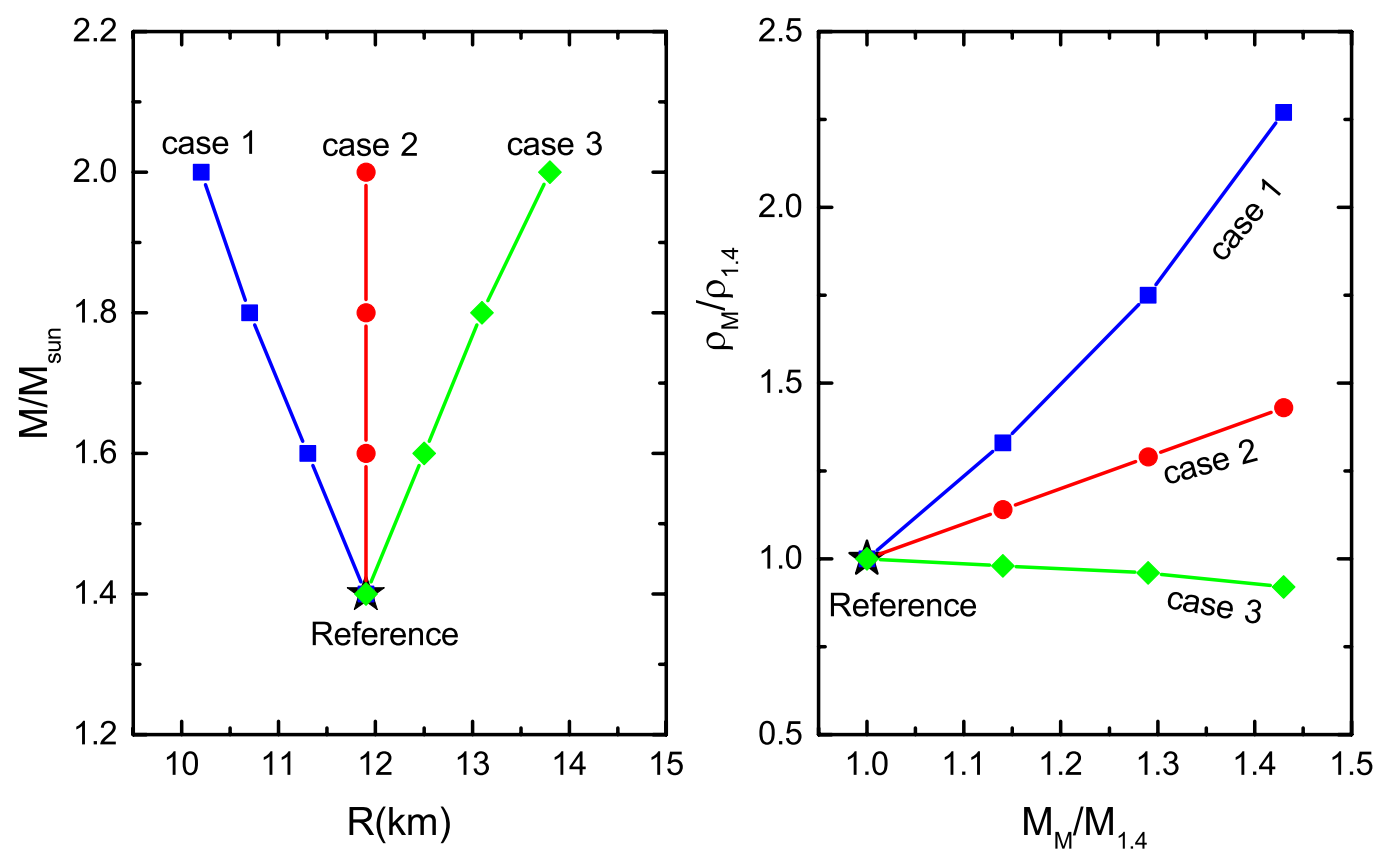

Figure 9. (Left) Representative mocked mass-radius correlations considered for massive NSs with respect to the reference of $R_{1.4}=11.9 \pm 1.4 \mathrm{~km}$ at a $90 \%$ confidence level for canonical NSs from GW170817. (Right) The corresponding average density in NSs of mass M scaled by that of canonical NSs as a function of the mass ratio $M / M_{1.4}$. Taken from [67].

It is seen that the PDFs of $L$ and $K_{\text {sym }}$ are strongly peaked compared to their uniform prior PDFs, leading to the extraction of $L=66_{-20}^{+12} \mathrm{MeV}$ and $K_{\text {sym }}=-120_{-100}^{+80} \mathrm{MeV}$ at a $68 \%$ confidence level. As discussed in detail in Reference [67], the peak of $J_{0}$ is mainly due to the requirement to support NSs with masses at least as massive as $1.97 M_{\odot}$, while the considered NS data have little effect on constraining $K_{0}$ or $E_{\text {sym }}\left(\rho_{0}\right)$, indicated by their very similar prior and posterior PDFs. This is because these two parameters characterize only the properties of neutron-rich matter at saturation density. On the other hand, the $J_{\text {sym }}$ parameter characterizes the behavior of $E_{\mathrm{sym}}(\rho)$ at densities above $2 \rho_{0}$. It is seen that the posterior PDF of $J_{\text {sym }}$ peaks at its upper boundary and would keep changing as its prior range changes, indicating that the NS data used do not constrain this parameter (thus the behavior of $E_{\mathrm{sym}}(\rho)$ at densities higher than about $2 \rho_{0}$, consistent with the findings from the direction inversion approach shown in Figure 4). Again, this is mainly because the radii of canonical NSs are determined mainly by the nuclear pressure around $2 \rho_{0}$. They are not sensitive to the symmetry energy at higher densities, while the NS mass is mostly determined by the SNM EOS, unless the symmetry energy becomes super-soft, as we shall discuss.

To see the impacts of the constraints on $L$ and $K_{\text {sym }}$ from the above analyses, shown on the left of Figure 12 is $K_{\text {sym }}$ as a function of $L$ for 33 unified neutron star EOSs from Fortin et al. [117] in comparison with their $68 \%$ confidence boundaries from analyzing neutron observables (Xie and Li) [67], as well as terrestrial experiments and theoretical calculations for the PNM EOS (Newton and Crocombe) [118]. The 33 unified EOSs were derived by Fortin et al. [117] within the Skyrme-Hartree-Fock and the RMF models for the core and the Thomas-Fermi model for the crust using the same interactions. Only seven of them are in the overlapping area of the two constraints used. Shown in the right window are the mass-radius correlations predicted by these seven EOSs in comparison with the latest observational constraints. It is seen that the KDE0v1 EOS is further excluded by the mass 2.14 $M_{\odot}$ of MSP J0740+6620, as Xie and Li's constraint was derived by using an NS minimum mass of $1.97 \mathrm{M}_{\odot}$, as in the original LIGO/VIRGO data analysis of GW170817. Thus, it is clear that the constraints on $L$ and $K_{\text {sym }}$ from analyzing the observables of 
neutron stars help greatly in screening theoretical predictions for the EOS of dense neutronrich matter.
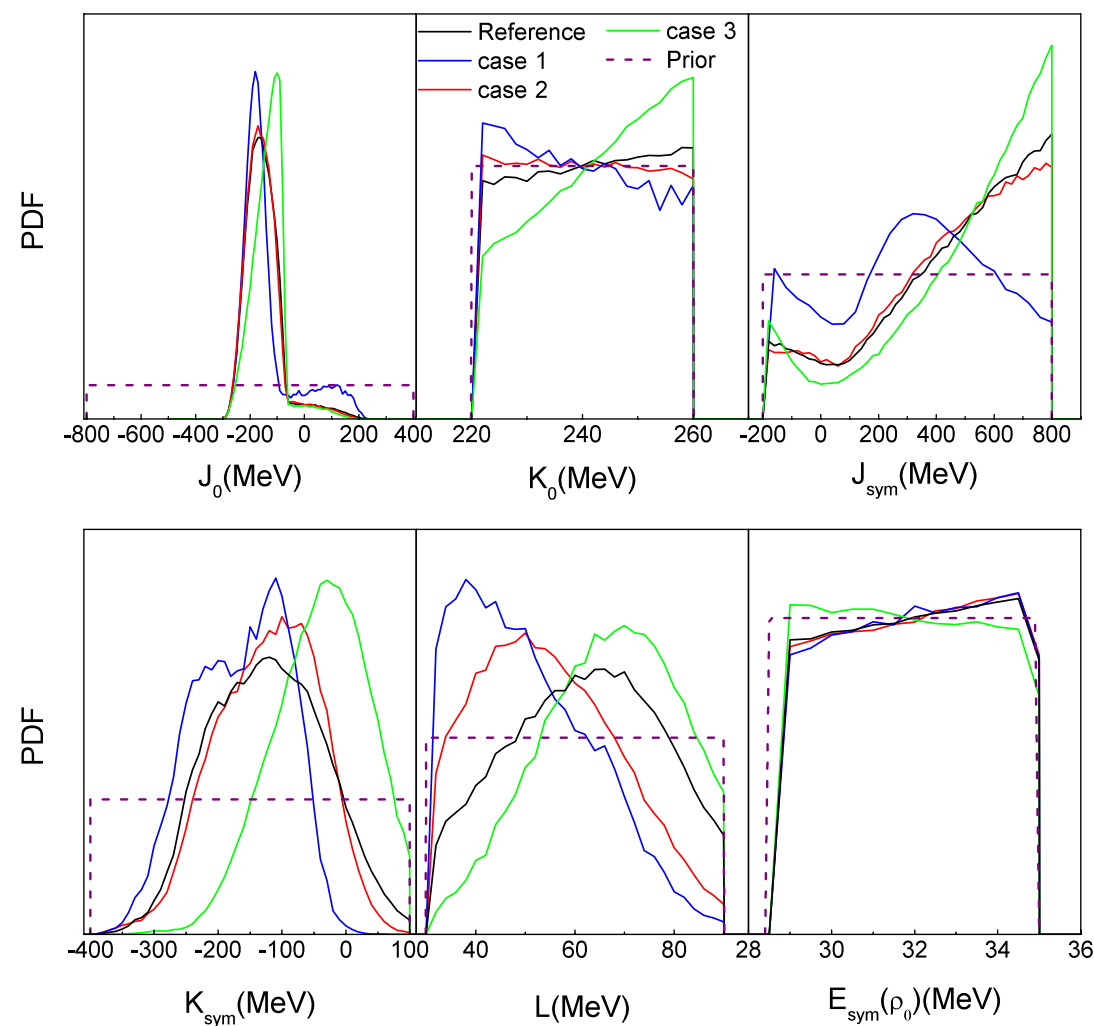

Figure 10. The posterior PDFs of the 6 EOS parameters in comparison with their prior PDFs for the three cases using the mass-radius data shown in Figure 9. Taken from [67].

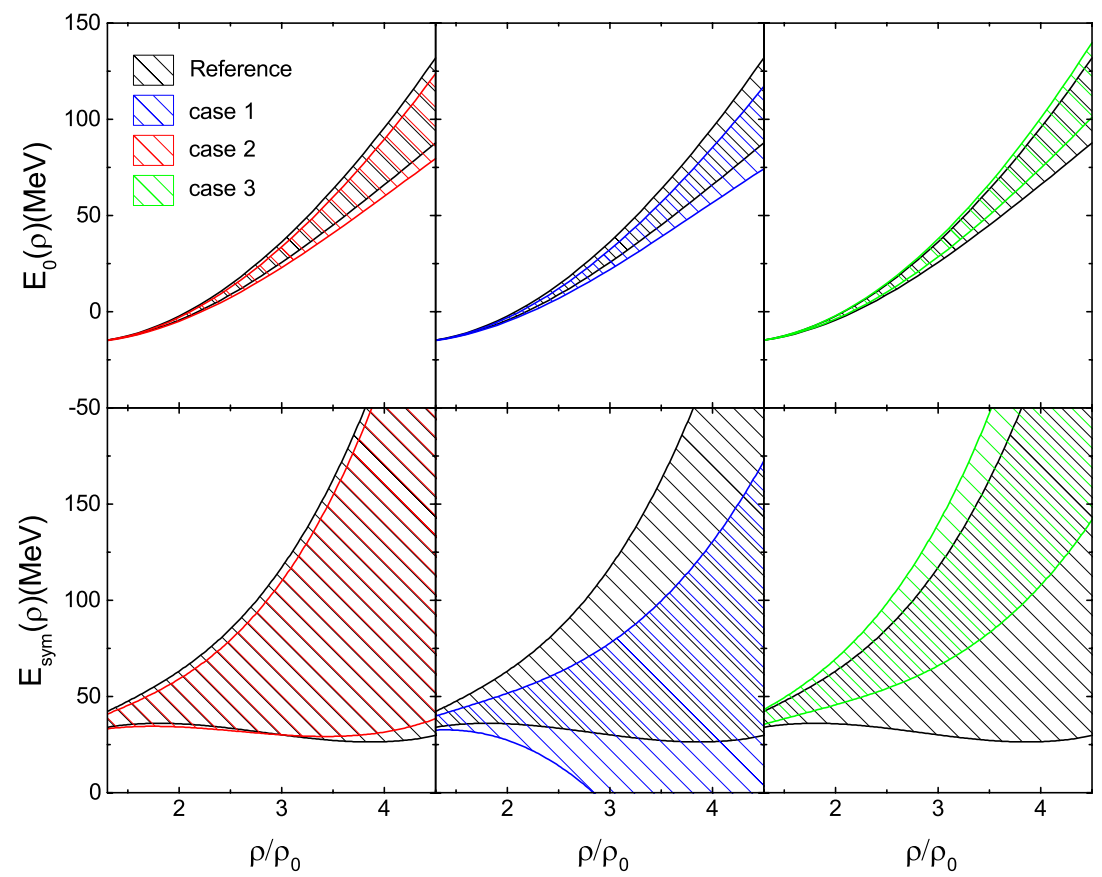

Figure 11. The $68 \%$ boundaries of nuclear symmetry symmetry (bottom) and SNM EOS (top) inferred from the neutron star radius data shown in Figure 9. Taken from [67]. 

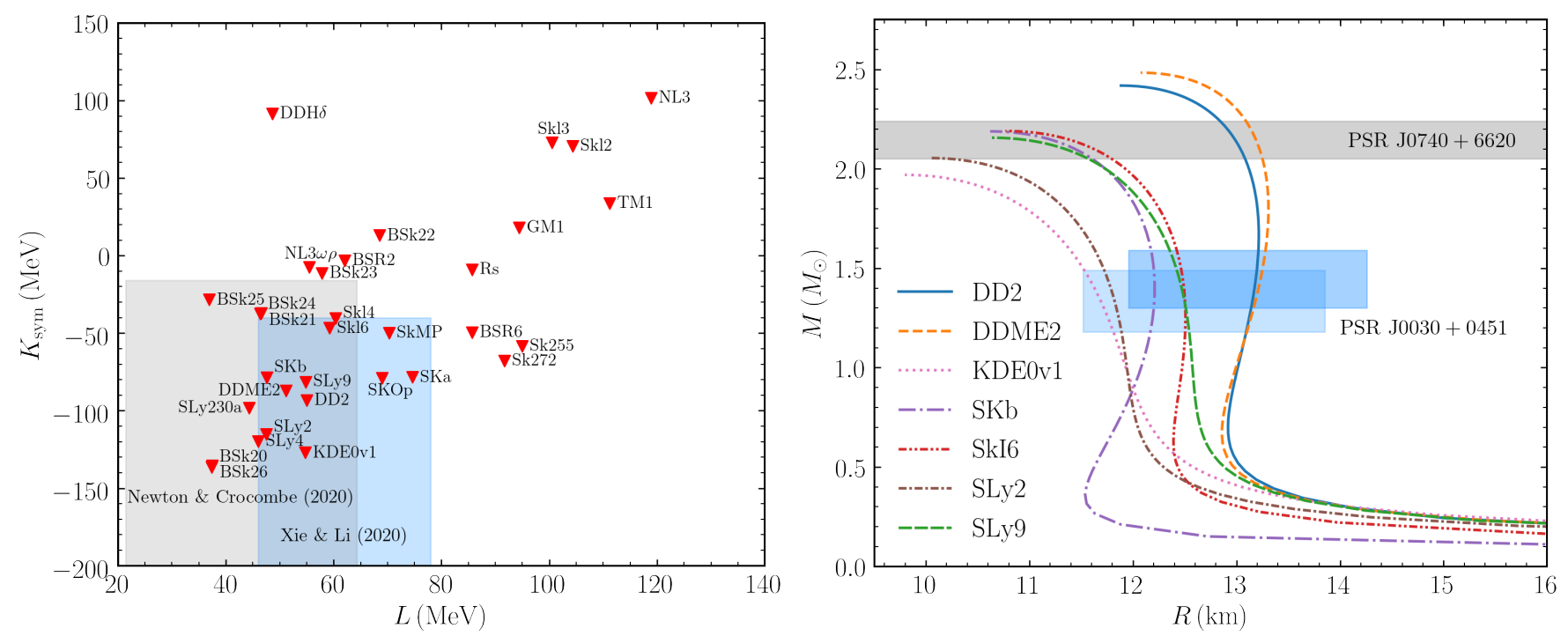

Figure 12. (Left) $K_{\text {sym }}$ versus $L$ for 33 unified neutron star EOSs from Fortin et al. [117] in comparison with their $68 \%$ confidence boundaries from analyzing neutron observables (Xie and Li) [67], as well as terrestrial experiments and theoretical calculations for the PNM EOS (Newton and Crocombe) [118]. (Right) Mass-radius correlations of the EOSs filtered by the $K_{\text {sym }}$ versus $L$ constraints in comparison with the observational constraints. Taken from [43].

\subsection{Future Radius Measurements of Massive Neutron Stars and Their Constrains on High-Density Nuclear Symmetry Energy}

Will future high-precision radius measurements of more massive NSs help constrain the symmetry energy at densities above $2 \rho_{0}$ ? This question was studied in [67]. In the following, we summarize the main findings with a few illustrations. To answer this question, three sets of mock mass-radius correlations were used in a comprehensive Bayesian analysis, as shown in Figure 9. They all start from the same reference point of $R_{1.4}=11.9 \pm 1.4 \mathrm{~km}$ and use the same uncertainty of $1.4 \mathrm{~km}$ at a $90 \%$ confidence level for all data points. The scaled average densities in NSs for these three typical mass-radius relations are shown in the right window of Figure 9, assuming NSs are made of neutrons, protons, electrons, and muons only. It is seen that for an NS of $2.0 M_{\odot}$, its average density scaled by that in an NS of $1.4 M_{\odot}$ is significantly different in the three cases. In particular, in Case 2, the scaled density increases by about $50 \%$ from 1.4 to $2.0 M_{\odot}$. The recent report by Miller et al. indicated that NICER observations favor a mass-radius relation between Case 2 and Case 3 [108]. As mentioned earlier, NICER found the mass of $1.44_{-0.14}^{+0.15} M_{\odot}$ and $R=13.02_{-1.06}^{+1.24} \mathrm{~km}$ for PSR J0030+0451 [104] and $R=12.2-16.3 \mathrm{~km}$ for PSR J0740+6620 with an updated mass of $2.08 \pm 0.07 M_{\odot}$ [108].

A systematic Bayesian inference of the EOS parameters using the mocked mass-radius data discussed above was carried out in [67]. The resulting PDFs of the EOS parameters are compared in Figure 10. In Case 2, where the radius is independent of the mass, the resulting PDFs of all six EOS parameters are not much different from those of the reference (a single canonical NS with a radius of $R_{1.4}=11.9 \pm 1.4 \mathrm{~km}$ ), although the average density increases by about $50 \%$ from 1.4 to $2.0 M_{\odot}$. Nevertheless, comparing the PDFs from Case 1 and Case 3 , all PDFs change significantly. There are also some secondary bumps and dips due to the correlations among the EOS parameters, as discussed in [67]. The resulting $68 \%$ confidence boundaries of the symmetry energy (bottom) and SNM EOS (top) are shown in Figure 11 in comparison with the results of the reference case. Most interestingly, while the confidence boundaries of SNM EOSs are not much different in all three mock cases and the reference case as all EOSs are required to support the same NS minimum mass of $1.97 M_{\odot}$, the symmetry energy boundaries especially at high densities are quite different from Case 1 to Case 3 . This indicates that the radii of massive NSs will help constrain the symmetry energy at densities above $2 \rho_{0}$, where it is most uncertain, with little influence from the remaining uncertainties of high-density SNM EOS. We noticed again that the lower boundary of symmetry energy 
at high densities had appreciable dependence on the NS minimum maximum mass $\mathrm{M}_{\max }$ used. In the results shown, $\mathrm{M}_{\max }=1.97 \mathrm{M}_{\odot}$ was used. If it were replaced by $2.01 M_{\odot}$ or even higher, the lower boundaries were expected to move up.

How important is the precision of radius measurements? What happens if the precision is not high enough to distinguish the three possible mass-radius correlations shown in Figure 9? To answer these questions, shown in Figure 13 are comparisons of the PDFs of the six EOS parameters calculated for Case 2 discussed above and a new calculation using a constant radius $R_{\mathrm{M}}=R_{1.4}=11.9 \pm 3.2 \mathrm{~km}$ at a $90 \%$ confidence level for all NSs considered. Thus, the two data sets have the same mean radius, but the new case has an error bar that is three times that of Case 2. It is so large that one can no longer distinguish the three cases shown in Figure 9. As one would expect, the posterior PDFs of all three symmetry energy parameters, especially for the slope $L$ and curvature $K_{\text {sym }}$, became significantly wider. In fact, the $L$ parameter can now go higher than its prior upper limit used. On the other hand, it was seen that the PDFs of the SNM EOS parameters were basically the same as the masses, and the mean radii of the NSs considered were kept the same. Therefore, the precision of the radius measurement for massive neutron stars can significantly affect the accuracy of constraining the symmetry energy, but not greatly the EOS of SNM.
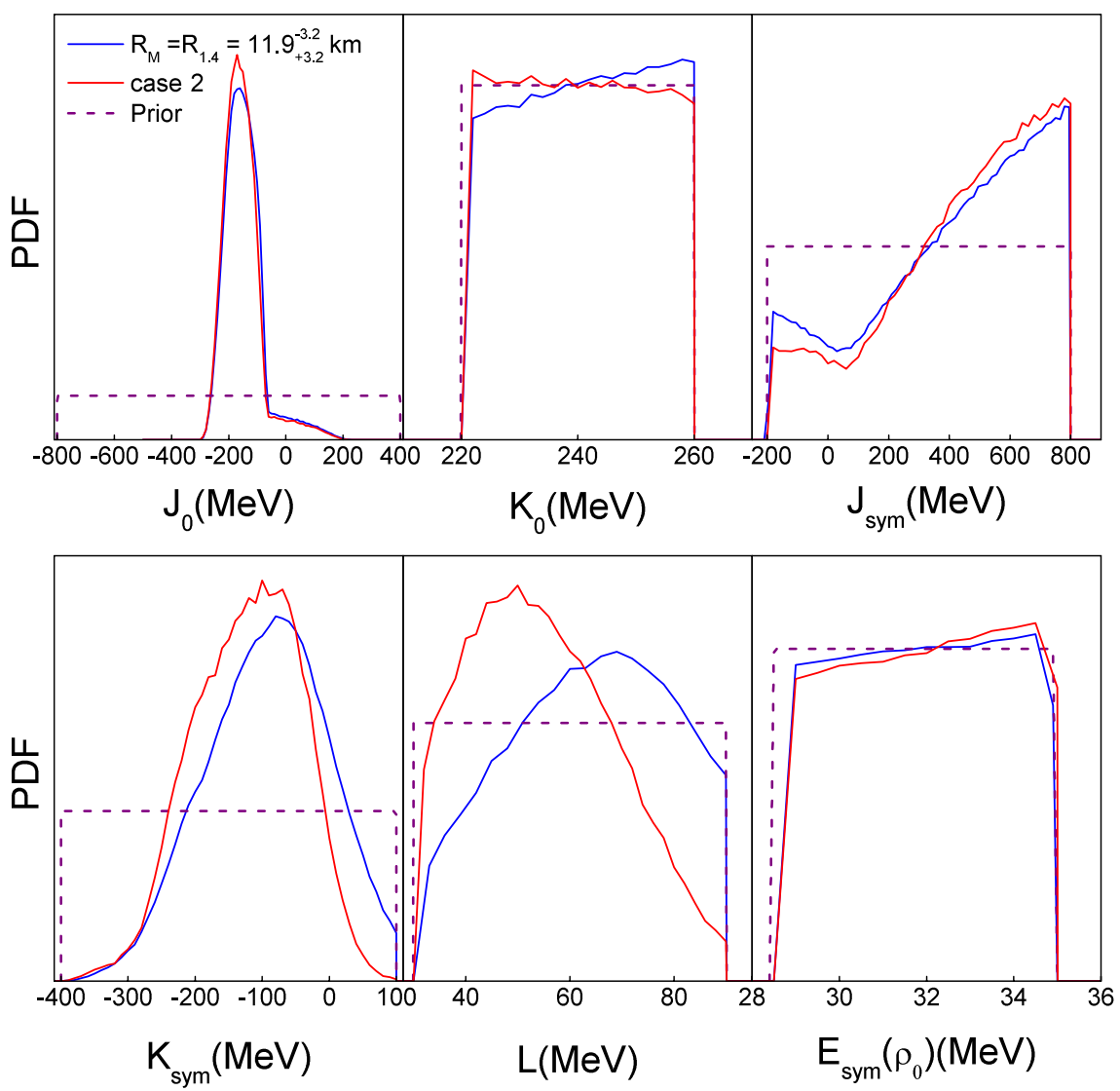

Figure 13. Prior and posterior probability distribution functions of EOS parameters assuming all neutron stars have the same radius of $R_{\mathrm{M}}=R_{1.4}=11.9 \pm 3.2 \mathrm{~km}$ in comparison with the results from Case 2, which has the same mean radius of $11.9 \mathrm{~km}$, but an error bar of $1.4 \mathrm{~km}$ at a $90 \%$ confidence level (black curves shown in Figure 10). Taken from [67].

\section{Effects of Hadron-Quark Phase Transition on Extracting Nuclear Symmetry Energy from Neutron Star Observables}

Whether Quark Matter (QM) exists and how big its mass or volume may be are among the longstanding and interesting issues regarding the structure of neutron stars [119]. In the forward modeling of neutron stars, often, one assumes a hadron-quark phase transition density around several times the saturation density of nuclear matter. To the best of 
knowledge, there is still a hadron-quark duality in understanding NS observables, namely there is so far no unique signature for the existence of quark matter in NSs, while highfrequency post-merger gravitational waves from collisions of NSs were predicted to provide more clear signatures of hadron-quark phase transition in neutron star matter [120,121]. Both the purely hadronic matter and the hybrid hadron-quark mixture can equally describe all available NS observations. In the literature, most of the studies on nuclear symmetry energy using NS observables were carried out using the purely hadronic picture. Therefore, how does the consideration of possible hadron-quark phase transition in NSs affect the extraction of high-density symmetry energy? This question was studied recently in [122]. Here, we summarize the main findings from this study.

\subsection{Bayesian Inference of Hadronic and Quark Matter EOS Parameters from Observations of Canonical Neutron Stars}

In [122], a nine-parameter metamodel was used to generate both hadronic and quark matter EOSs in the Markov chain Monte Carlo sampling process within the Bayesian statistical framework. For the hadronic phase, the six-parameter EOS was constructed using Equations (5) and (6). It was connected through the Maxwell construction to the Constant Speed of Sound (CSS) model for quark matter developed by Alford, Han, and Prakash [123]. More specifically, the pressure can be written as:

$$
\varepsilon(p)=\left\{\begin{array}{ll}
\varepsilon_{\mathrm{HM}}(p) & p<p_{t} \\
\varepsilon_{\mathrm{HM}}\left(p_{t}\right)+\Delta \varepsilon+c_{\mathrm{QM}}^{-2}\left(p-p_{t}\right) & p>p_{t}
\end{array},\right.
$$

where $\varepsilon_{\mathrm{HM}}(p)$ is the Hadronic Matter (HM) EOS below the hadron-quark transition pressure $p_{t}, \Delta \varepsilon$ is the discontinuity in energy density $\varepsilon$ at the transition, and $c_{\mathrm{QM}}$ is the QM speed of sound.

In the Bayesian analyses using the metamodel EOS described above, besides the causality condition and the requirement that all EOSs have to be stiff enough to support NSs at least as massive as $2.14 \mathrm{M}_{\odot}$, the following radii of canonical NSs were used as independent data: (1) $R_{1.4}=11.9 \pm 1.4 \mathrm{~km}$ from GW170817 by the LIGO/VIRGO Collaborations [103], (2) $R_{1.4}=10.8_{-1.6}^{+2.1}$ extracted independently also from GW170817 by De et al. [116], (3) $R_{1.4}=11.7_{-1.1}^{+1.1}$ from earlier analysis of quiescent low-mass X-ray binaries [105], and (4) $R=13.02_{-1.06}^{+1.24} \mathrm{~km}$ with mass $M=1.44_{-0.14}^{+0.15} M_{\odot}$ [104] or $R=12.71_{-1.85}^{+1.83} \mathrm{~km}$ with mass $M=1.34 \pm 0.24 M_{\odot}$ [112] for PSR J0030+0451 from the NICER Collaboration. The errors quoted are at the $90 \%$ confidence level.

Shown in Figure 14 are the resulting posterior PDFs and correlations of quark matter EOS parameters $\rho_{t} / \rho_{0}, \Delta \varepsilon / \varepsilon_{t}$, and $c_{\mathrm{QM}}^{2} / c^{2}$, as well as the fraction $f_{\mathrm{QM}}^{\text {mass }}$ (defined as the ratio of QM mass over the total NS mass) and the radius of quark matter $R_{\mathrm{QM}}$. Interestingly, the most probable hadron-quark transition density $\rho_{t} / \rho_{0}=1.6_{-0.4}^{+1.2}$ was found to be rather low, while the transition strength $\Delta \varepsilon / \varepsilon_{t}=0.4_{-0.15}^{+0.20}$ was modest and the speed of sound in $\mathrm{QM} c_{\mathrm{QM}}^{2} / c^{2}=0.95_{-0.35}^{+0.05}$ very high. The latter is understandable as the most probable transition density is very low, so the stiffness of QM EOS represented by its $c_{\mathrm{QM}}^{2}$ value has to be high enough to support the neutron star with a large QM core. In fact, the strong anti-correlation between the transition density and speed of sound in QM is clearly shown in their correlation function shown. Since the average baryon density in a canonical NS with a $12 \mathrm{~km}$ radius is about $2 \rho_{0}$ (compatible with the low transition density), the QM fraction and its radius are both quite high. We noticed that the rather low hadron-quark transition density found in [122] was also found very recently in two independent Bayesian analyses using similar NS data [124,125]. Thus, based on these Bayesian analyses, a large volume of QM exists even in canonical NSs. However, it was found in [126] that it is unlikely to form a quark core in canonical neutron stars. 


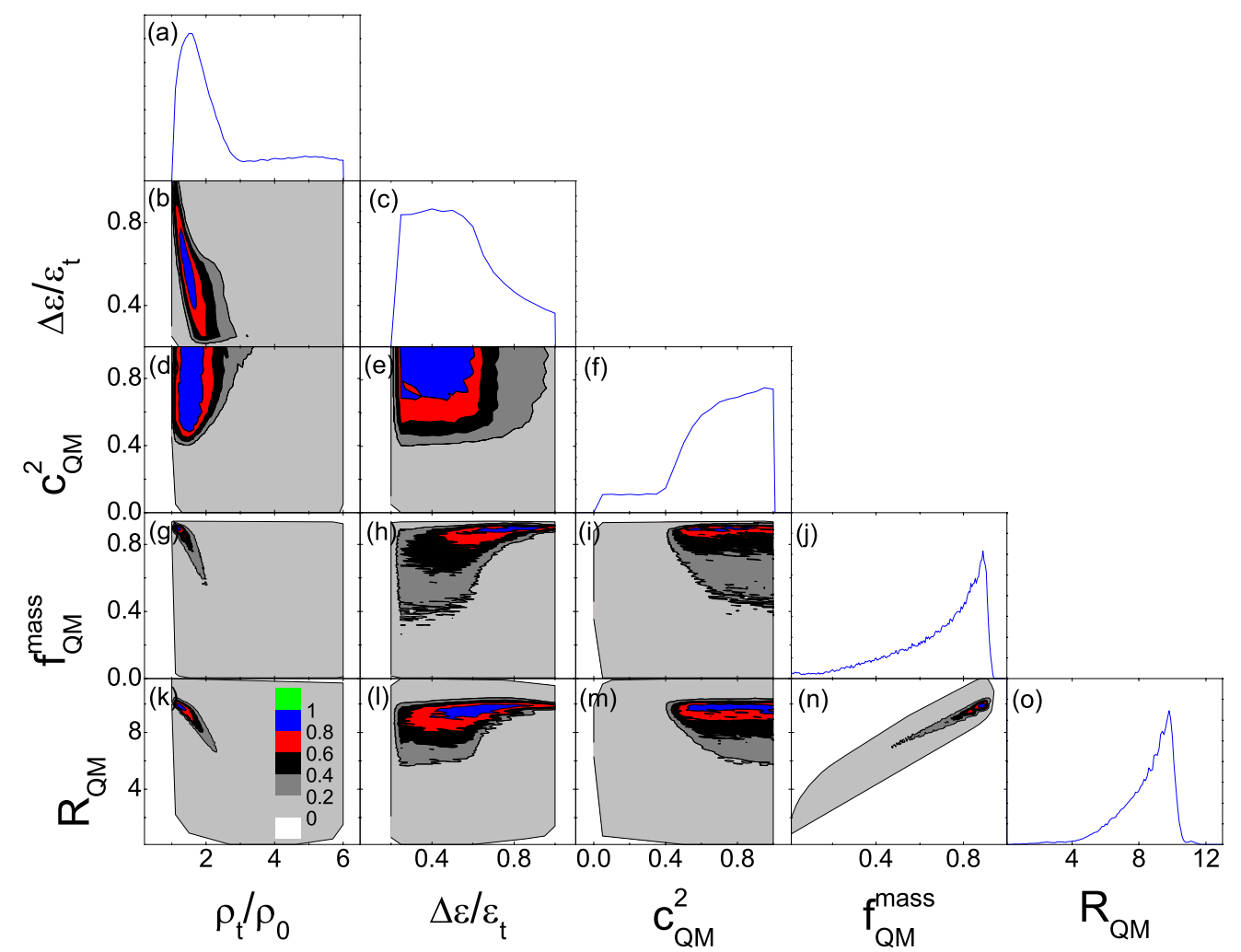

Figure 14. The subfigures along the diagonal are the posterior probability distribution functions (in arbitrary units) of the five quantities labeled (the three quark matter EOS parameters, as well as the fraction and radius of quark matter in hybrid neutron stars of mass $1.4 M_{\odot}$ ). See text for the definitions of each quantity shown. All off-diagonal subfigures are the correlations among the 5 quantities labeled. Taken from [122].

3.2. Comparing Symmetry Energy Parameters from Analyzing Neutron Star Observables with and without Considering the Hadron-Quark Phase Transition

How does the consideration of the hadron-quark phase transition affect the extraction of nuclear symmetry energy from NS observables? To answer this question, shown in the left window of Figure 15 are comparisons of the PDFS of six hadronic EOS parameters with and without considering the hadron-quark phase transition, while in the right are comparisons of the corresponding $68 \%$ confidence boundaries of the SNM EOS (top) and symmetry energy (bottom).

It is seen that while the incompressibility $K_{0}$ and symmetry energy $E_{\text {sym }}\left(\rho_{0}\right)$ at saturation density $\rho_{0}$ are not much different, as one expects, the PDF of $J_{0}$ characterizing the stiffness of SNM at suprasaturation densities shifts towards higher values as the hadron-quark phase transition softens the EOS, unless $c_{\mathrm{QM}}^{2} / c^{2}$ is very high. The hadronic EOS needs to be stiffened to support the same NSs. Furthermore, with the hadron-quark phase transition, three additional parameters in the CSS model are used. The $68 \%$ confidence boundaries of the SNM EOS become wider, as shown in the upper right window. Thus, with the PDF of $\rho_{t} / \rho_{0}$ peaks at $1.6_{-0.4}^{+1.2}$, in the model with the hadron-quark phase transition, the posterior PDFs of $L$ and $K_{\text {sym }}$ shift significantly higher to reproduce the same radius data, while the poster PDF of $J_{\text {sym }}$ is not much different from its prior PDF. The latter is, however, significantly different from the PDF in the case without considering the hadron-quark phase transition. Thus, as shown in the lower right window, the high-density behavior of nuclear symmetry energy extracted from NS observables is quite different with or without considering the hadron-quark phase transition. With three more parameters introduced in the CSS model, the uncertainty of the high-density symmetry energy becomes larger, similar to the SNM EOS. Nevertheless, the symmetry energy extracted is about the same 
at densities less than about $2 \rho_{0}$, as one expects from the most probable transition density given above.
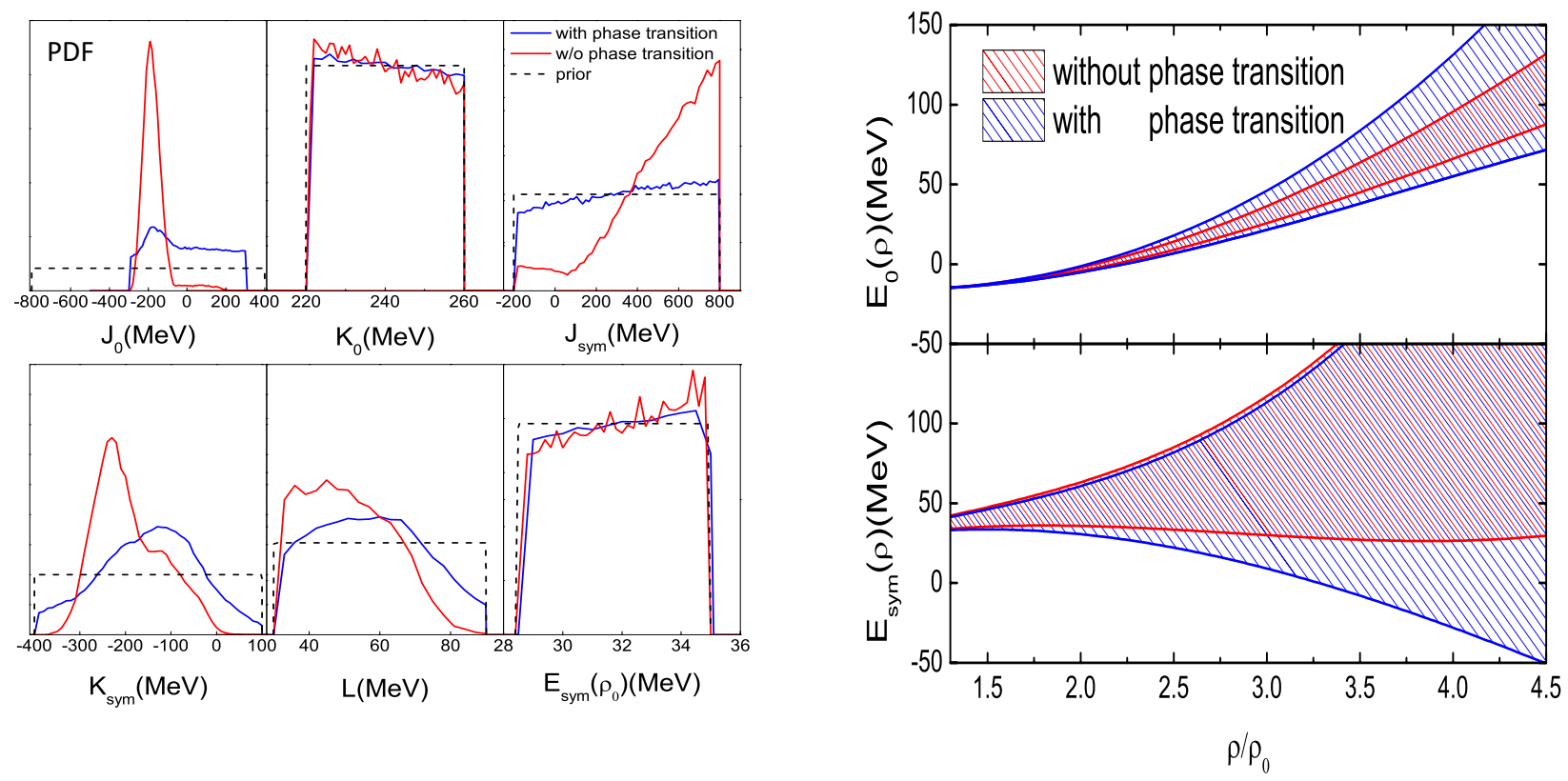

Figure 15. (Left) The posterior probability distribution functions (in arbitrary units) of nuclear matter EOS parameters inferred from Bayesian analyses with (thick blue curves) and without (thin red curves) considering the hadron-quark phase transition in neutron stars in comparison with their uniform priors (dashed curves). (Right) The corresponding $68 \%$ confidence boundaries of SNM EOS (top) and symmetry energy (bottom). Taken from [122].

\section{Effects of Symmetry Energy on the Second Component of GW190814 as a Supermassive and Superfast Pulsar}

Recently, the LIGO/Virgo Collaborations reported the binary merger event of GW190814: the coalescence of a (22.2-24.3) $M_{\odot}$ black hole with a (2.50-2.67) $M_{\odot}$ compact object [127]. This event generated much excitement and interest partially because: (1) The mass of the secondary falls into the mass gap range $\left(\sim 2-5 M_{\odot}\right)$ where $\sim 5 M_{\odot}$ is the smallest observed/predicted mass of black holes (see, e.g., [128,129]) and $\sim 2 M_{\odot}$ is the largest mass observed/predicted of neutron stars (i.e., 2.14 ${ }_{-0.10}^{+0.11} M_{\odot}$ for PSR J0740+6620 [106] or its recently revised value of $2.03_{-0.08}^{+0.10} M_{\odot}$ [130] when analyzed using a populationinformed prior). (2) The highly asymmetric mass ratio and large merger rate of the GW190814-type class of binaries are hard to explain. Different from GW170817 [131], no tidal effects in the signal and electromagnetic counterpart to the gravitational waves have been measured/identified. Therefore, whether the secondary is a massive neutron star, low-mass black hole, or an exotic object is still under hot debate. Determining the nature of GW190814's secondary can potentially help identify the mass boundary between neutron star and black hole and update models of stellar evolution (see, e.g., [132-134]).

\subsection{Is GW190814's Secondary a Superfast and Supermassive Neutron Star or Something Else?}

Many possible mechanisms leading to the GW190814 event and the related natures of its secondary component have been proposed in a flood of interesting papers in the literature recently. It is certainly beyond our knowledge range to review all of these interesting works. To the best of our knowledge, most analyses indicated that GW190814's secondary is a neutron star, while many other works suggested it as a black hole or an exotic object. For example, Abbott et al. [127] initially explained it as a black hole with a probability larger than 97\% using the GW170817-informed spectral EOS samples [103]. Applying Bayesian analyses, Essick \& Landry [135] and Tews et al. [136] found that GW190814's secondary is a binary black hole merger with a probability of $>94 \%$ and $>99.9 \%$, respectively. 
Moreover, Fattoyev et al. [137], Das et al. [138] found that the requirement of a very stiff EOS to support $2.5 M_{\odot}$ neutron stars is inconsistent with either constraints obtained from analyzing energetic heavy-ion collisions or the low deformability of medium-mass neutron stars. While Li et al. [139], Sedrakian et al. [140] considered the $\Delta$-resonance and hyperons, they found that their results were inconsistent with a stellar nature interpretation of GW190814's secondary, implying that this event involved two black holes rather than a neutron star and a black hole.

On the other hand, the possibility for GW190814's secondary as a neutron star can be accomplished by: (1) choosing/constructing stiff EOSs having a maximum mass larger than $2.5 M_{\odot}$ [76,141-150]; (2) considering the effects of fast rotations, which can increase the maximum mass by about $20 \%$ when a star rotates at the Kepler frequency (the maximum frequency at which the gravitational attraction is still sufficient to keep matter bound to the pulsar surface) [42,43,139,140,145,151-157]; (3) considering other effects/models that can modify the maximum mass of a neutron star, such as the magnetic field [147], twin star [158], two families of compact stars [142], finite temperature [153], antikaon condensation [159], net electric charge [144], etc.

Since fast rotation is among the easiest mechanisms to increase the masses of NSs, rotational effects have been considered in several model frameworks for investigating the nature of GW190814's secondary. For example, adopting a maximum mass $\mathrm{M}_{\mathrm{TOV}}=2.3 \mathrm{M}_{\odot}$ for non-rotating NSs, Most et al. [154] found that GW190814's secondary does not need to be an ab initio black hole, nor an exotic object. Rather, it can be a fast pulsar collapsed to a rotating $\mathrm{BH}$ at some point before the merger. Riahi \& Kalantari [155] calculated the maximum mass at Kepler frequency with four DDRMF EOSs. Two of them can support pulsars heavier than $2.5 \mathrm{M} \odot$ after considering rotation. Dexheimer et al. [152] discussed rotating hybrid stars within a Chiral Mean Field (CMF) model. They can generate stellar masses that approach, and in some cases surpass, $2.5 M_{\odot}$. It was shown that in such cases, fast rotation does not necessarily suppress exotic degrees of freedom due to changes in stellar central density, but requires a larger amount of baryons than what is allowed in the non-rotating stars. This is not the case for pure quark stars, which can easily reach $2.5 M_{\odot}$ and still possess approximately the same amount of baryons as stable non-rotating stars. Khadkikar et al. [153] selected 11 EOSs from relativistic density functional theories, Skyrme functionals, and an empirical extension of a variational microscopic model. Hyperons were also included in their discussions. It was shown that rotation can easily increase the maximum mass to larger than $2.5 \mathrm{M} \odot$. On the other hand, Tsokaros et al. [157] showed that one can find EoSs that both satisfy the constraints of GW170817 and at the same time imply that GW190814's secondary component is a non-rotating NS.

Among the studies supporting GW190814's secondary component as a supermassive and superfast pulsar, the required rotational frequencies were found to be rather high. In fact, most of the studies found that the minimum frequencies are significantly higher than the fastest known pulsar PSR J1748-2446ad with a frequency of $716 \mathrm{~Hz}$ [160], thus making GW190814's secondary the most massive and fastest known pulsar if its nature is confirmed. For example, Most et al. [154] found that a rotation frequency of $1210 \mathrm{~Hz}$ is needed assuming GW190814's secondary has a typical radius of $12.5 \mathrm{~km}$ and a $M_{\mathrm{TOV}}=2.08 \mathrm{M}_{\odot}$ when it is not rotating. Zhang \& $\mathrm{Li}$ [42] found a minimum rotation frequency of $971 \mathrm{~Hz}$ and an equatorial radius of $11.9 \mathrm{~km}$ using a model EOS that predicts a $M_{\mathrm{TOV}}=2.39 M_{\odot}$. More recently, Biswas et al. [161] derived a minimum frequency of $1143_{-155}^{+194} \mathrm{~Hz}$ and an equatorial radius $R_{e}=15.7_{-1.7}^{+1.0} \mathrm{~km}$ at the $90 \%$ confidence level assuming $M_{\mathrm{TOV}}=2.14 M_{\odot}$. In another study, Demircik et al. [151] investigated a hybrid star based on the APR EOS for hadronic matter and the V-QCD model for quark matter. The maximum mass can reach about $2.9 \mathrm{M}_{\odot}$. However, even with the stiff EOS model, high rotation frequencies $\geq 1 \mathrm{kHz}$ are required to reach $2.5 \mathrm{M}_{\odot}$. 


\subsection{Is GW190814's Secondary r-Mode Stable If It Is Really a Superfast Pulsar?}

It is well known that fast pulsars could be r-model unstable (see, e.g., [162] for a review), leading to the exponential growth of Gravitational Wave (GW) emission through the Chandrasekhar-Friedman-Schutz mechanism $[163,164]$ if the GW growth rate is faster than its damping rate. It is known that a rigid crust provides the strongest r-mode damping, and it can well explain the stability of all observed Low-Mass X-ray Binaries (LMXBs) [43], albeit that some people may consider this extreme mechanism unrealistic.

Is GW190814's secondary r-mode stable? This question was recently addressed by Zhou et al. [43] using the six EOSs shown in Figure 12 that meet all current constraints from both astrophysics and nuclear physics and the rigid crust damping mechanism. It was found that five of them can support pulsars with masses higher than $2.5 M_{\odot}$ if they rotate faster than the minimum frequencies $v_{\min }$ listed in Table 1 . Shown also in the table are the maximum mass of non-rotating neutron stars $M_{\mathrm{TOV}}$ and Kepler frequency $v_{\mathrm{K}}$ for the five EOSs used. $v_{\min }$ is plotted as a horizontal line in Figure 16, where the r-mode stability boundary for each EOS is shown in the frequency-temperature plane. Their cross point is marked with a diamond, indicating the maximum temperatures $T_{\max }$ below which neutron stars remain r-mode stable. The values of $T_{\max }$ are listed as the fifth column of Table 1 . It is seen that GW190814's secondary is r-mode stable as long as its temperature is sufficiently low, e.g., lower than about $3.9 \times 10^{7} \mathrm{~K}$ when it is rotating at $1169.6 \mathrm{~Hz}(0.744$ times its Kepler frequency). Because this temperature is about an order of magnitude higher than that of some known old neutron stars, it was thus concluded that GW190814's secondary can be r-mode stable depending on its temperature [43].

Table 1. The maximum mass of non-rotating neutron stars $M_{\mathrm{TOV}}$, the Kepler frequency $v_{\mathrm{K}}$, and the minimum frequency $v_{\min }$ to support a neutron star of mass $2.50 \mathrm{M}_{\odot}$ for the 5 EOSs used. $T_{\max }$ is the maximum temperature for the $m_{2}$ to remain r-mode stable. Taken from [43].

\begin{tabular}{ccccc}
\hline Model & $\boldsymbol{M}_{\text {TOV }}\left(\mathbf{M}_{\odot}\right)$ & $\boldsymbol{v}_{\mathbf{K}} \mathbf{( H z )}$ & $\boldsymbol{v}_{\min }\left(v_{\mathbf{K}}\right)$ & $\boldsymbol{T}_{\max }\left(\mathbf{1 0}^{\mathbf{7}} \mathbf{K}\right)$ \\
\hline DD2 & 2.42 & 1197 & 0.76 & 3.0 \\
DDME2 & 2.48 & 1170 & 0.74 & 3.9 \\
SKb & 2.20 & 1447 & 0.81 & 1.3 \\
SkI6 & 2.20 & 1433 & 0.83 & 1.1 \\
SLy9 & 2.16 & 1515 & 0.86 & 0.75 \\
\hline
\end{tabular}

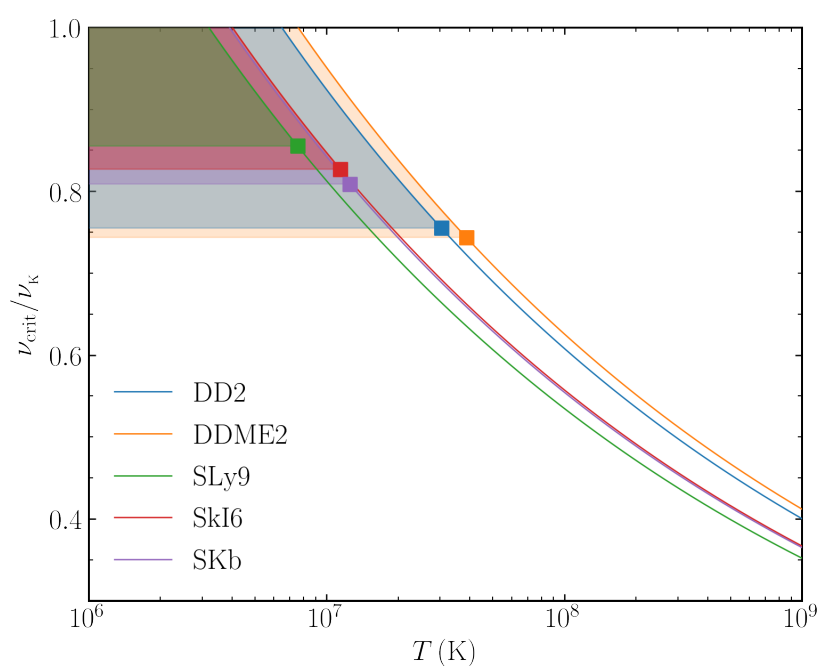

Figure 16. The minimum frequency $v_{\min }$ to support a neutron star of mass $2.50 M_{\odot}$ is plotted as a horizontal line in the frequency-temperature plane where the r-mode stability boundary for each EOS is also shown. Their cross point is marked with a diamond indicating the maximum temperatures $T_{\max }$ below which neutron stars remain r-mode stable. Taken from [43]. 
4.3. Effects of Symmetry Energy on the Mass, Radius, and Minimum Rotation Frequency of GW190814's Secondary Component as a Supermassive and Superfast Pulsar

Why is the high-density nuclear symmetry energy important for determining the nature of GW190814's secondary component besides its know effects on the structure of neutron stars as we discussed earlier? Basically, the answer lies in the fact that the maximum mass of non-rotating neutron stars $M_{\mathrm{TOV}}[96]$, the Kepler frequency $v_{\mathrm{K}}[165,166]$, and the r-mode stability boundaries $[167,168]$ are all known to depend sensitively on the high-density behavior of nuclear symmetry energy; for a review, see, e.g., [11]. For example, shown in the left window of Figure 17 are the maximum masses $M_{\mathrm{TOV}}$ while that in the right window are the corresponding radii $R_{\max }$ of non-rotating NSs. They were obtained by solving TOV equations for the EOS parameter sets on the causality surface (the blue surface in the left panel of Figure 4) as functions of the curvature $K_{\text {sym }}$ and skewness $J_{\text {sym }}$ of nuclear symmetry energy. All other EOS parameters used to calculate the $M_{\mathrm{TOV}}$ and $R_{\max }$ surfaces are the same as those used in Figure 4 , i.e., $E_{\text {sym }}\left(\rho_{0}\right)=31.6 \mathrm{MeV}$ and $L=58.9 \mathrm{MeV}$. The $M_{\mathrm{TOV}}$ on the causality surface represents the absolutely maximum mass allowed for non-rotating neutron stars. For a comparison, the $2.01 \mathrm{M}_{\odot}$ mass plane is also shown in the left window. The space below this surface is excluded.
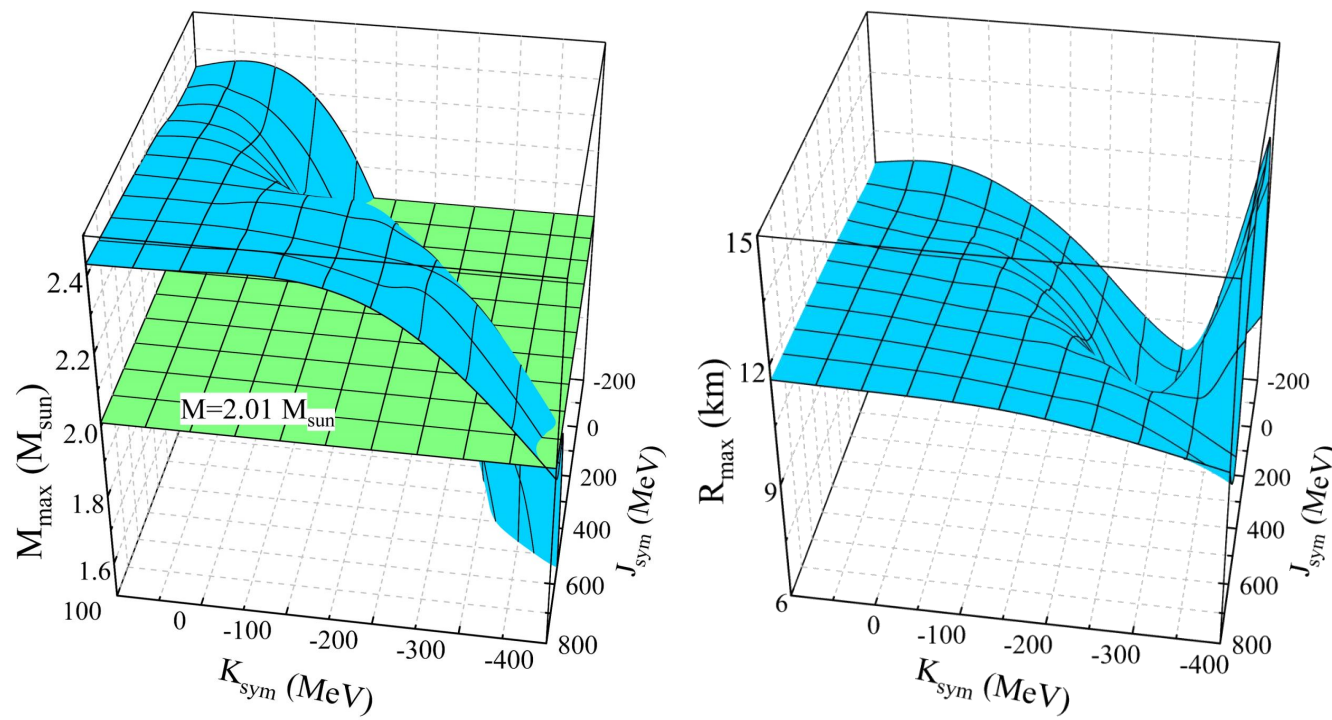

Figure 17. The maximum masses $M_{\mathrm{TOV}}$ (left) and corresponding radii (right) of non-rotating NSs on the causality surface as functions of the curvature and skewness of nuclear symmetry energy. Taken from [96].

As we discussed earlier, large positive (negative) $K_{\text {sym }}$ and $J_{\text {sym }}$ represent stiff (soft) high-density symmetry energies. Correspondingly, the interiors of neutron stars are less (more) neutron-rich from the energy consideration or due to the so-called isospin fractionation $[11,25]$. Thus, for the stiff high-density symmetry energy, the pressure is dominated by that of SNM EOS. It is thus seen that $M_{\mathrm{TOV}}$ flattens towards an asymptotic value of about $2.39 \mathrm{M}_{\odot}$, determined mostly by the $J_{0}$ of SNM EOS when the high-density symmetry energy becomes very stiff. The corresponding radius reaches a constant of about $12 \mathrm{~km}$. On the other hand, when the high-density symmetry energy becomes very soft, the interior of neutron stars becomes very neutron-rich. Then, the contribution to the nuclear pressure from the high-density symmetry energy becomes more important, but can be even negative, thus reducing the total nuclear pressure. Consequently, $M_{\mathrm{TOV}}$ becomes even smaller than $2.01 M_{\odot}$. The corresponding radius also becomes smaller.

Depending on what $M_{\mathrm{TOV}}$ one uses for non-rotating neutron stars, the minimum frequency necessary to rotationally support neutron stars heavier than $2.50 M_{\odot}$ will thus be different. As an example, shown in Figure 18 are the mass-radius relations of both static (solid curves) and rotating neutron stars at Kepler frequencies (dashed curves) with the 12 
selected EOSs along and/or inside the symmetry energy boundaries shown in the right window of Figure 4 . We note that $M_{\mathrm{TOV}}$ on the bounded causality surface is between 2.14 and $2.39 M_{\odot}$. Neutron stars rotating at the minimum frequency $f_{2.5}$ that can rotationally support a neutron star with mass $2.50 M_{\odot}$ are shown with dotted curves. The reported mass $2.50-2.67 M_{\odot}$ of GW190814's secondary component is shown as gray bands.

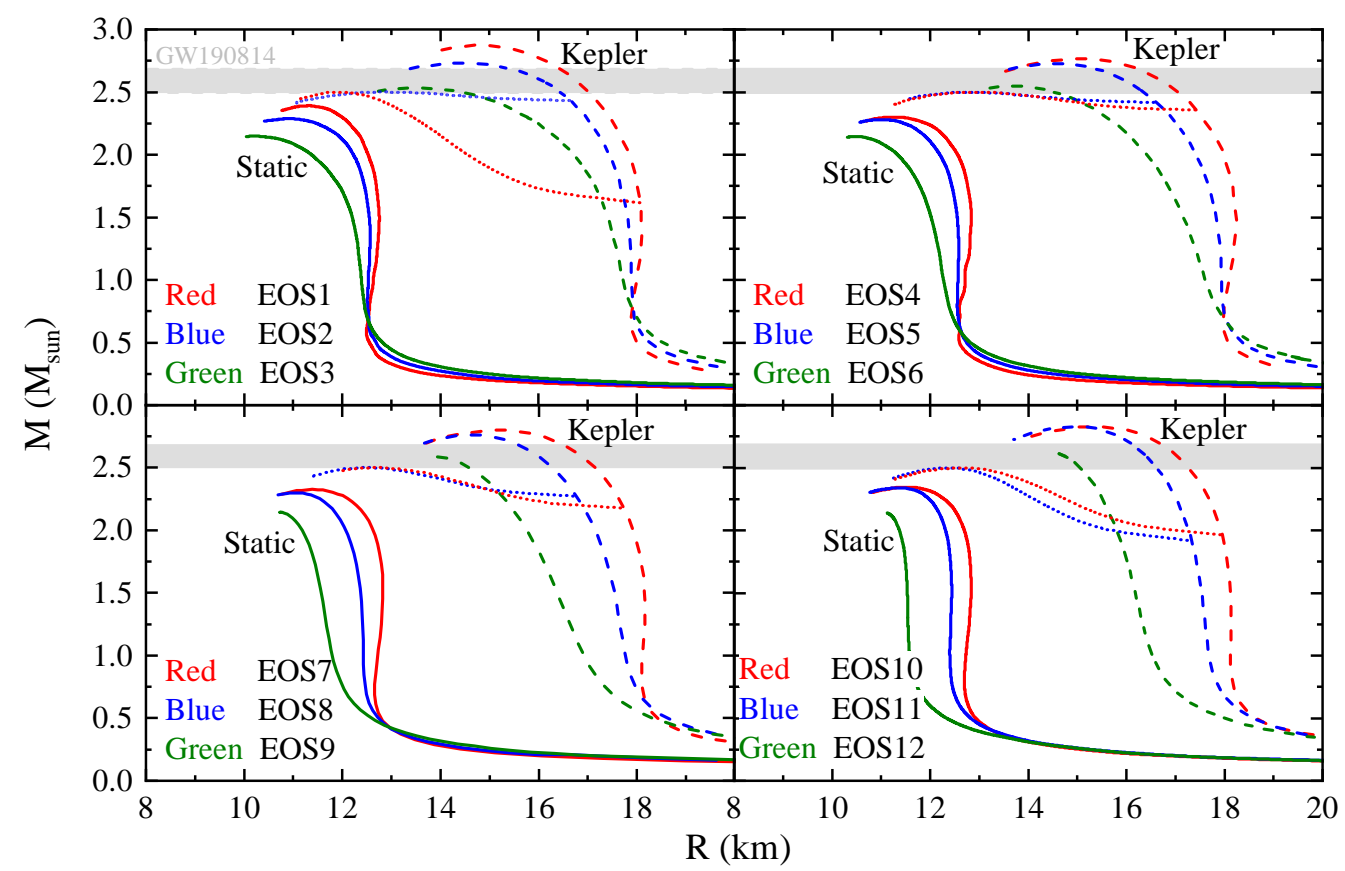

Figure 18. Mass-radius relations of both static (solid lines) and rotating neutron stars with the selected EOSs along the symmetry energy boundaries shown in the right window of Figure 4 . The NSs rotating at the Kepler frequencies and minimum frequency that can rotationally support a NS with mass $2.5 M_{\odot}$ are shown as dashed and dotted lines, respectively. Taken from [42].

It is interesting to see that while the $\mathrm{M}_{\mathrm{TOV}}$ of the 12 EOSs are between 2.14 and $2.39 M_{\odot}$, pulsars at their respective Kepler frequencies can easily sustain masses heavier than $2.50 M_{\odot}$. For example, with the stiffest EOS possible (EOS1 with $M_{\mathrm{TOV}}=2.39 M_{\odot}$ ), the maximum rotating mass is $2.87 M_{\odot}$, while with the soft EOSs including EOS3, EOS6, EOS9, and EOS12 on the right boundary of the allowed EOS space shown in Figure 4, the maximum rotating masses are slightly higher than $2.5 M_{\odot}$, but less than $2.67 M_{\odot}$. As the maximum mass of these EOSs is close to $2.5 M_{\odot}, f_{2.5}$ is slightly lower than the Kepler frequency; thus, the RNS code [169] does not output the $f_{2.5}$ for EOS3, EOS6, EOS9, and EOS12.

One can measure the minimum frequency necessary to support the pulsar with mass $2.50 M_{\odot}$ with the dimensionless spin parameter $\chi_{2.5}=J / M^{2}$ where $J$ is the angular momentum of the pulsar of mass $\mathrm{M}=2.50 \mathrm{M}_{\odot}$. Shown in the left window of Figure 19 is the density dependence of nuclear symmetry energy (upper) with different skewness $J_{\text {sym }}$ parameters and the corresponding isospin asymmetry $\delta$ of neutron star matter at $\beta$-equilibrium (lower). The latter clearly shows how the high-density symmetry energy affects the composition of neutron stars at $\beta$-equilibrium, i.e., their neutron richness.

As discussed earlier, the upper limit of nuclear symmetry is determined by the crossline between the causality surface and the $R_{1.4}=12.83 \mathrm{~km}$ surface (or the nearby $\Lambda_{1.4}=580$ surface) before NICER's observation for the radius of PSR J0740+6620. This crossline was projected to the $K_{\text {sym }}-J_{\text {sym }}$ plane and shown as the left boundary in the right panel of Figure 4. In Figure 18, only four selected EOSs (EOS1, EOS4, EOS7, and EOS10) along this boundary are used. To show the $M_{\mathrm{TOV}}$ of non-rotating NSs along this boundary more clearly, $M_{\mathrm{TOV}}$ as a continuous function of $J_{\text {sym }}$ is now shown in the upper-right 
window of Figure 4. For a comparison, the previously reported mass $M=2.14_{-0.09}^{+0.10} M_{\odot}$ of PSR J0740+6620 is also shown in the upper panel. The resulting EOSs of neutron star matter along the boundary discussed above are the stiffest while being consistent with all known astrophysical and nuclear constraints. The resulting minimum spin parameter $\chi_{2.5}$ is shown in the lower right window as a function of the skewness parameter $J_{\text {sym }}$. The arrows indicate the conditions for GW190814's secondary to be a superfast pulsar. Clearly, as we mentioned earlier, the minimum spin parameter $\chi_{2.5}$ depends on $M_{\mathrm{TOV}}$, and both of them depend on $J_{\text {sym }}$ characterizing the high-density behavior of nuclear symmetry energy.
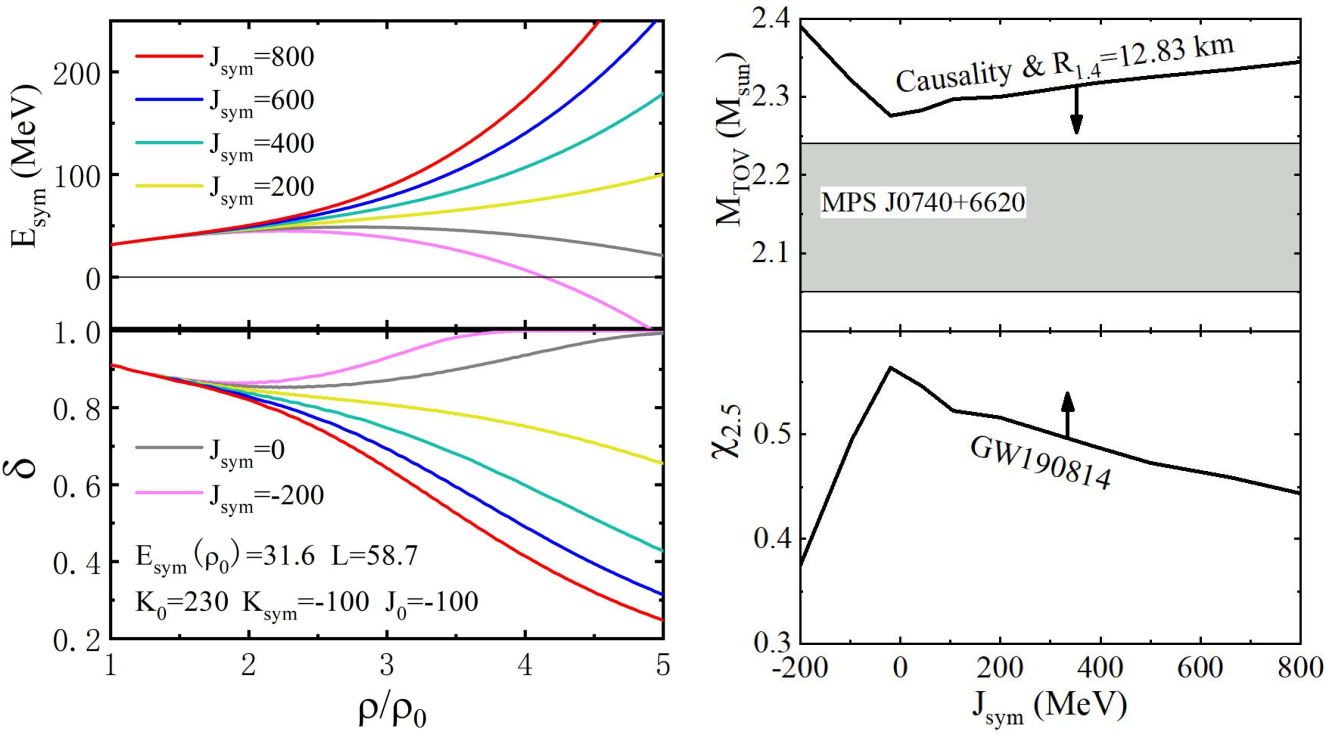

Figure 19. (Left) The density dependence of nuclear symmetry energy (upper) and the corresponding isospin asymmetry (lower) in neutron stars at $\beta$ equilibrium with different skewness $J_{\text {sym }}$ parameters. (Right) The maximum mass $M_{\mathrm{TOV}}$ of non-rotating NSs (upper window) and the minimum spin parameter $\chi_{2.5}$ of pulsars with the frequency $f_{2.5}$ (lower window) as functions of the skewness parameter of nuclear symmetry energy parameter. The previously observed NS maximum mass $M=2.14_{-0.09}^{+0.10} M_{\odot}(68 \%$ confidence level $)$ of MSR J0740+6620 is shown in the upper panel. The arrows indicate the conditions for GW190814's secondary to be a superfast pulsar. Taken from [42].

5. An Auxiliary Function Approach for Predicting the High-Density Behavior of Nuclear Symmetry Energy Based on Its Slope $L$, Curvature $K_{\text {sym }}$, and Skewness $J_{\text {sym }}$ at $\rho_{0}$

Assuming now that the community has finally reached a consensus about the characteristics of nuclear symmetry energy at $\rho_{0}$, i.e., $L, K_{\mathrm{sym}}$, and $J_{\mathrm{sym}}$ are all well determined using various approaches, how can we use these quantities to predict the symmetry energy at suprasaturation densities? While one may use the same theory/model used in extracting these parameters at $\rho_{0}$ to predict the high-density symmetry energy, it is well known that most of the theories/models are unreliable at high densities above about $2 \rho_{0}$. One may also try to extrapolate the symmetry energy at $\rho_{0}$ to high densities by using the conventional Taylor expansion, namely $E_{\text {sym }}(\rho) \approx S+L \chi+2^{-1} K_{\text {sym }} \chi^{2}+6^{-1} J_{\text {sym }} \chi^{3}+\cdots$. Unfortunately, the latter breaks down eventually as the density $\rho$ increases above the certainty limit because $\chi$ is not always small enough for small-quantity expansions to work properly. For example, the RMF model with the FSUGold parameters predicts exactly the symmetry energy $E_{\mathrm{sym}}(\rho)$ as a function of density in a broad density range. One can extract from the prediction the characteristics $L, K_{\text {sym }}$, and $J_{\text {sym }}$ of the symmetry energy at $\rho_{0}$. It was shown quantitatively in [60] that using these characteristics in the standard Taylor expansion up to the $\chi^{3}$ term, the resulting symmetry energy at densities above about $2 \rho_{0}$ deviates significantly from the exact $E_{\text {sym }}(\rho)$ function predicted by the RMF model, indicating the non-convergence of the Taylor expansion at high densities. Moreover, 
as we shall show, Taylor expansions up to the $\chi^{2}$ or $\chi^{3}$ term give significantly different predictions for $E_{\text {sym }}(\rho)$ at densities above $2 \rho_{0}$, indicating again the non-convergence of the Taylor expansion at high densities.

Therefore, is there a better way to predict the symmetry energy at $2-3 \rho_{0}$ using its characteristics at $\rho_{0}$ ? This question was recently studied in [60] using an auxiliary function approach. Here, we summarize the main idea and results.

\subsection{Theoretical Framework}

To predict accurately the symmetry energy at suprasaturation densities based on its known characteristics at $\rho_{0}$, such as those we surveyed in Section 2, the first task is to find an appropriate auxiliary function that naturally reproduces the first several terms given by the conventional expansion when it is expanded around $\chi=0$. Secondly, certain higher $\chi$ order contributions should also be effectively encapsulated in the auxiliary function using still only the first few characteristics of $E_{\mathrm{sym}}(\rho)$ at $\rho_{0}$, i.e., $L, K_{\mathrm{sym}}$, and $J_{\text {sym }}$. Mathematically, this was performed by introducing the auxiliary function $\Pi_{\text {sym }}\left(\chi, \Theta_{\text {sym }}\right)$, which itself is a function of the density $\rho$ (or equivalently of the $\chi$ ) and depends on a new parameter $\Theta_{\text {sym }}$. In expanding the symmetry energy, one has the following replacement,

$$
\frac{\mathrm{d}^{n} E_{\text {sym }}}{\mathrm{d} \rho^{n}}\left(\rho-\rho_{0}\right)^{n} \rightarrow \frac{\mathrm{d}^{n} E_{\text {sym }}}{\mathrm{d} \Pi_{\text {sym }}^{n}} \widetilde{v}_{\text {sym }}^{n}\left(\chi, \Theta_{\text {sym }}\right),
$$

where $\widetilde{v}_{\text {sym }}\left(\chi, \Theta_{\text {sym }}\right)=\Pi_{\text {sym }}\left(\chi, \Theta_{\text {sym }}\right)-\Pi_{\text {sym }}\left(0, \Theta_{\text {sym }}\right)$ corresponds to the dimensionless quantity $\chi$. Once a model $\Pi_{\text {sym }}\left(\chi, \Theta_{\text {sym }}\right)$ is adopted/selected, the parameter $\Theta_{\text {sym }}$ can be determined by the symmetry energy at a density where it is well determined experimentally.

Given the four characteristic parameters $S \equiv E_{\text {sym }}\left(\rho_{0}\right), L, K_{\text {sym }}$, and $J_{\text {sym }}$ of $E_{\text {sym }}(\rho)$ at $\rho_{0}$, the symmetry energy $E_{\text {sym }}(\rho)$ can be expanded around $\Pi_{\text {sym }}\left(\chi, \Theta_{\text {sym }}\right)=\Pi_{\text {sym }}\left(0, \Theta_{\text {sym }}\right)$ to order $v_{\mathrm{sym}}^{3}\left(\chi, \Theta_{\mathrm{sym}}\right)$ as:

$$
E_{\text {sym }}(\rho) \approx S+L v_{\text {sym }}\left(\chi, \Theta_{\text {sym }}\right)+\frac{1}{2} K_{\text {sym }} \Phi v_{\text {sym }}^{2}\left(\chi, \Theta_{\text {sym }}\right)+\frac{1}{6} J_{\text {sym }} \Psi v_{\text {sym }}^{3}\left(\chi, \Theta_{\text {sym }}\right),
$$

where:

$$
\begin{aligned}
\Phi=1 & +\frac{L}{K_{\text {sym }}}\left(\frac{1}{3 \rho} \frac{\partial^{2} \rho}{\partial \Pi_{\text {sym }}^{2}}\right) /\left(\frac{1}{3 \rho} \frac{\partial \rho}{\partial \Pi_{\text {sym }}}\right)_{\chi=0}^{2}, \\
\Psi=1 & +\frac{K_{\text {sym }}}{J_{\text {sym }}}\left(\frac{1}{3 \rho^{2}} \frac{\partial \rho}{\partial \Pi_{\text {sym }}} \frac{\partial^{2} \rho}{\partial \Pi_{\text {sym }}^{2}}\right) /\left(\frac{1}{3 \rho} \frac{\partial \rho}{\partial \Pi_{\text {sym }}}\right)_{\chi=0}^{3} \\
& +\frac{L}{J_{\text {sym }}}\left(\frac{1}{3 \rho} \frac{\partial^{3} \rho}{\partial \Pi_{\text {sym }}^{3}}\right) /\left(\frac{1}{3 \rho} \frac{\partial \rho}{\partial \Pi_{\text {sym }}}\right)_{\chi=0}^{3},
\end{aligned}
$$

and,

$$
v_{\text {sym }}\left(\chi, \Theta_{\text {sym }}\right) \equiv\left[\frac{1}{3 \rho} \frac{\partial \rho}{\partial \Pi_{\text {sym }}\left(\chi, \Theta_{\text {sym }}\right)}\right]_{\chi=0} \cdot \widetilde{v}_{\text {sym }}\left(\chi, \Theta_{\text {sym }}\right),
$$

It was shown that the conventional Taylor expansion corresponds to the special case of selecting $\Pi_{\text {sym }}\left(\chi, \Theta_{\text {sym }}\right) \propto \chi$. Moreover, terms higher than $\chi^{3}$ are effectively included in Equation (9), although it is truncated at order $v_{\text {sym }}^{3}\left(\chi, \Theta_{\text {sym }}\right)$, since the latter itself encapsulates the higher order effects in $\chi$.

In [60], an exponential (abbreviated as "exp") and an algebraic (abbreviated as "alge") auxiliary function were used. These functions and the corresponding expansions of symmetry energy can be written as: 


$$
\begin{aligned}
\exp : \quad v_{\mathrm{sym}}\left(\chi, \Theta_{\mathrm{sym}}\right)= & \frac{1}{3 \Theta_{\mathrm{sym}}}\left(1-e^{-3 \chi \Theta_{\mathrm{sym}}}\right) \\
E_{\mathrm{sym}}(\rho) \approx S+ & L v_{\mathrm{sym}}\left(\chi, \Theta_{\mathrm{sym}}\right)+\frac{1}{2} K_{\mathrm{sym}}\left(1+\frac{3 L}{K_{\mathrm{sym}}} \Theta_{\mathrm{sym}}\right) v_{\mathrm{sym}}^{2}\left(\chi, \Theta_{\mathrm{sym}}\right) \\
& +\frac{1}{6} J_{\mathrm{sym}}\left(1+\frac{9 K_{\mathrm{sym}}}{J_{\mathrm{sym}}} \Theta_{\mathrm{sym}}+\frac{18 L}{J_{\mathrm{sym}}} \Theta_{\mathrm{sym}}^{2}\right) v_{\mathrm{sym}}^{3}\left(\chi, \Theta_{\mathrm{sym}}\right), \\
\text { alge : } v_{\mathrm{sym}}\left(\chi, \Theta_{\mathrm{sym}}\right)= & \chi \frac{1+\Theta_{\mathrm{sym}}^{-1}}{1+3 \chi+\Theta_{\mathrm{sym}}^{-1}}, \\
E_{\mathrm{sym}}(\rho) \approx S+ & L v_{\mathrm{sym}}\left(\chi, \Theta_{\mathrm{sym}}\right)+\frac{1}{2} K_{\mathrm{sym}}\left(1+\frac{6 L}{K_{\mathrm{sym}}} \frac{1}{1+\Theta_{\mathrm{sym}}^{-1}}\right) v_{\mathrm{sym}}^{2}\left(\chi, \Theta_{\mathrm{sym}}\right) \\
& +\frac{1}{6} J_{\mathrm{sym}}\left[1+\frac{18 K_{\mathrm{sym}}}{J_{\mathrm{sym}}} \frac{1}{1+\Theta_{\mathrm{sym}}^{-1}}+\frac{54 L}{J_{\mathrm{sym}}}\left(\frac{1}{1+\Theta_{\mathrm{sym}}^{-1}}\right)^{2}\right] v_{\mathrm{sym}}^{3}\left(\chi, \Theta_{\mathrm{sym}}\right) .
\end{aligned}
$$

Take the exponential model as an example. Some new features emerge in Equation (14). Besides the conventional term $2^{-1} K_{\text {sym }}$, a new term $3 \Theta_{\text {sym }} L / K_{\text {sym }}$ (normalized by $2^{-1} K_{\text {sym }}$ ) contributes at order $v_{\text {sym }}^{2}\left(\chi, \Theta_{\text {sym }}\right)$. This term is generally sizable and cannot be thought as a perturbation. For small $\chi$, e.g., $\rho_{0} \lesssim \rho \lesssim 3 \rho_{0}$, one has:

$$
v_{\text {sym }}\left(\chi, \Theta_{\text {sym }}\right) \approx \chi-\frac{3}{2} \Theta_{\text {sym }} \chi^{2}+\frac{3}{2} \Theta_{\text {sym }}^{2} \chi^{3}-\frac{9}{8} \Theta_{\text {sym }}^{3} \chi^{4}+\frac{27}{40} \Theta_{\text {sym }}^{4} \chi^{5}-\cdots,
$$

which approaches zero as $\chi \rightarrow 0$. It is clear that the effects of $\chi^{4}$ or $\chi^{5}$ are effectively generated with the help of the function $\Pi_{\text {sym }}\left(\chi, \Theta_{\text {sym }}\right)$. Shown in Figure 20 is an illustration of how the characteristics $L, K_{\text {sym }}$, and $J_{\text {sym }}$ of symmetry energy at $\rho_{0}$ affect both directly and indirectly through the parameter $\Theta_{\text {sym }}$ the high-density symmetry energy at density $\rho_{\mathrm{f}}$. The flow indicates the dependence. The conventional dependence of $E_{\mathrm{sym}}\left(\rho_{\mathrm{f}}\right)$ on $K_{\text {sym }}$ and $J_{\text {sym }}$, namely $\chi_{\mathrm{f}}^{2} / 2$ and $\chi_{\mathrm{f}}^{3} / 6$ are indicated, where $\chi_{\mathrm{f}}=\left(\rho_{\mathrm{f}}-\rho_{0}\right) / 3 \rho_{0}$.

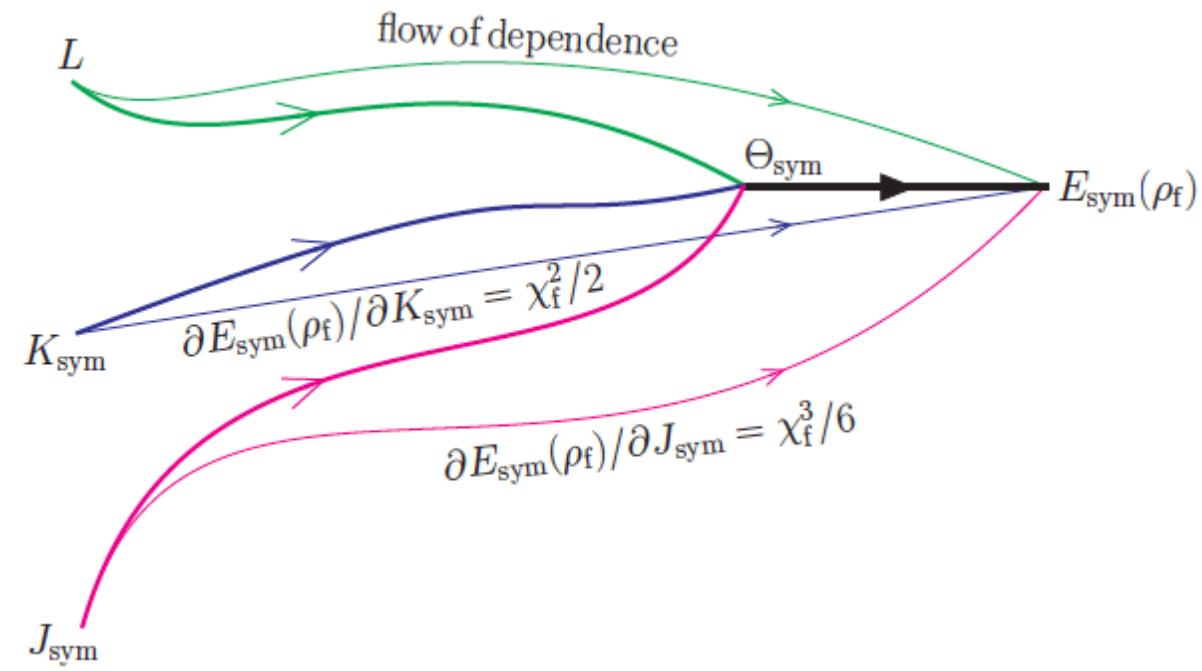

Figure 20. Illustration of how the characteristics $L, K_{\text {sym }}$, and $J_{\text {sym }}$ of symmetry energy at $\rho_{0}$ affect both directly and indirectly through the parameter $\Theta_{\text {sym }}$ the high-density symmetry energy at $\rho_{\mathrm{f}}$.

Several possible ways of determining the parameter $\Theta_{\text {sym }}$ were given in [60]. One approach is to fix it by using the experimentally known symmetry energy at some reference density. In particular, one can use the empirical constraint:

$$
E_{\mathrm{sym}}\left(\rho_{\mathrm{low}} \approx 0.05 \mathrm{fm}^{-3}\right) \approx 16.4 \pm 0.5 \mathrm{MeV}
$$


This is from analyzing both nuclear structures and reactions, e.g., isobaric analog states of several nuclei, the centroid energy of isovector giant dipole resonance, and electrical dipole polarizability of ${ }^{208} \mathrm{~Pb}$ [170-172].

By adopting the auxiliary-function-based reconstruction, one can easily find, e.g., in the exponential model, that the dependence of the symmetry energy at some density $\rho_{\mathrm{f}}$ on the characteristics $K_{\text {sym }}$ and $J_{\text {sym }}$ as,

$$
\begin{aligned}
& \frac{\partial E_{\text {sym }}\left(\rho_{\mathrm{f}}\right)}{\partial K_{\text {sym }}}=\frac{1}{2} v_{\text {sym }}^{\mathrm{f}, 2}\left(1+3 \Theta_{\text {sym }} v_{\text {sym }}^{\mathrm{f}}\right) \times\left[1-\left(\frac{v_{\text {sym }}^{\text {low }}}{v_{\text {sym }}^{\mathrm{f}}}\right)^{2}\left(\frac{1+3 \Theta_{\text {sym }} v_{\text {sym }}^{\text {low }}}{1+3 \Theta_{\text {sym }} v_{\text {sym }}^{\mathrm{f}}}\right)\left(\frac{\mathrm{Y}_{\mathrm{f}}}{\mathrm{Y}_{\text {low }}}\right)\right], \\
& \frac{\partial E_{\text {sym }}\left(\rho_{\mathrm{f}}\right)}{\partial J_{\text {sym }}}=\frac{1}{6} v_{\text {sym }}^{\mathrm{f}, 3} \times\left[1-\left(\frac{v_{\text {sym }}^{\text {low }}}{v_{\text {sym }}^{\mathrm{f}}}\right)^{3}\left(\frac{\mathrm{Y}_{\mathrm{f}}}{\mathrm{Y}_{\text {low }}}\right)\right],
\end{aligned}
$$

where the superscripts/subscripts " $\mathrm{f}$ " and "low" are for $\rho_{\mathrm{f}}$ and $\rho_{\text {low }} \approx 0.05 \mathrm{fm}^{-3}$, respectively. The function $\mathrm{Y}(\rho)$ in the above two equations is given by:

$$
\begin{aligned}
\mathrm{Y}(\rho)= & \frac{3}{2} L v_{\mathrm{sym}}^{2}+\frac{3}{2}\left(K_{\mathrm{sym}}+4 L \Theta_{\mathrm{sym}}\right) v_{\mathrm{sym}}^{3} \\
& +\frac{\partial v_{\mathrm{sym}}}{\partial \Theta_{\mathrm{sym}}}\left[L+\left(K_{\mathrm{sym}}+3 L \Theta_{\mathrm{sym}}\right) v_{\mathrm{sym}}+\frac{1}{2}\left(J_{\mathrm{sym}}+9 K_{\mathrm{sym}} \Theta_{\mathrm{sym}}+18 L \Theta_{\mathrm{sym}}^{2}\right) v_{\mathrm{sym}}^{2}\right] .
\end{aligned}
$$

The corrections in the square brackets in Equations (19) and (20) come from the dependence of the $\Theta_{\text {sym }}$ parameter on the curvature $K_{\text {sym }}$ and the skewness $J_{\text {sym }}$ of the symmetry energy, i.e., $\partial \Theta_{\text {sym }} / \partial K_{\text {sym }}$ and $\partial \Theta_{\text {sym }} / \partial J_{\text {sym }}$, as sketched in Figure 20 . In the conventional expansion, one has:

$$
\frac{\partial E_{\text {sym }}\left(\rho_{\mathrm{f}}\right)}{\partial K_{\mathrm{sym}}}=\frac{1}{2} \chi_{\mathrm{f}}^{2}, \frac{\partial E_{\mathrm{sym}}\left(\rho_{\mathrm{f}}\right)}{\partial J_{\mathrm{sym}}}=\frac{1}{6} \chi_{\mathrm{f}}^{3} .
$$

By comparing Equation (19) with the first relation of Equation (22) or Equation (20) with the second relation of Equation (22), one can immediately find that if the new expansion element $v_{\text {sym }}$ is constructed reasonably, the dependence of $E_{\text {sym }}\left(\rho_{\mathrm{f}}\right)$ on $K_{\text {sym }}$ or $J_{\text {sym }}$ at high densities should be reduced, as compared with the conventional approach, where the expansion element $\chi$ is unbounded from above.

\subsection{An Example of Applications}

To illustrate the advantages of the auxiliary function approach over the Taylor expansion in predicting the symmetry energy at high densities, shown in Figure 21 are comparisons of the symmetry energies from the two approaches from Monte Carlo simulations performed in [60]. The three test sets have different characteristic parameters for $E_{\text {sym }}(\rho)$ at $\rho_{0}$ : (I) $E_{\text {sym }}(\rho)$ is expanded to order $v_{\text {sym }}^{2}$ or order $\chi^{2}$ with $-300 \mathrm{MeV} \leq \mathrm{K}_{\text {sym }} \leq 0 \mathrm{MeV}$ [67]; (II) $E_{\text {sym }}(\rho)$ is expanded to order $v_{\text {sym }}^{3}$ or order $\chi^{3}$ with $-300 \mathrm{MeV} \leq \mathrm{K}_{\text {sym }} \leq 0 \mathrm{MeV}, 0 \mathrm{MeV} \leq$ $\mathrm{J}_{\text {sym }} \leq 2000 \mathrm{MeV}$; (III) $E_{\text {sym }}(\rho)$ is expanded to order $v_{\text {sym }}^{3}$ or order $\chi^{3}$ with $K_{\text {sym }}$ and $J_{\text {sym }}$ given by the intrinsic relations imposed by the unbound nature of PNM [173],

$$
\begin{aligned}
K_{\text {sym }} & \approx K_{0}\left(1-\frac{1}{3} \frac{K_{0}}{L}+\frac{1}{2} \frac{J_{0}}{K_{0}} \frac{L}{K_{0}}\right), \\
J_{\text {sym }} & \approx \frac{2 K_{0}^{3}}{3 L^{2}}\left(1-\frac{3 L}{K_{0}}\right)+\frac{I_{0} L}{3 K_{0}}+\left(\frac{2 K_{0} K_{\text {sym }}}{L}-J_{0}\right)\left(1+\frac{J_{0} L}{K_{0}^{2}}-\frac{K_{\text {sym }}}{K_{0}}\right) .
\end{aligned}
$$

For these demonstrations, the magnitude $S \approx 32 \pm 4 \mathrm{MeV}$ and slope $L \approx 60 \pm$ $30 \mathrm{MeV}$ of symmetry energy at $\rho_{0}$ [61], the incompressibility $K_{0} \approx 240 \pm 40 \mathrm{MeV}$ [174-178], skewness $J_{0} \approx-300 \pm 200 \mathrm{MeV}$ [179], and kurtosis $I_{0} \approx 0 \pm 2000 \mathrm{MeV}$ of SNM were adopted in [60]. The $\Theta_{\text {sym }}$ parameter in Set I was found to be about $\Theta_{\text {sym }} \approx 1.67 \pm 0.56$, while that in Set II (Set III) was found to be about $\Theta_{\text {sym }} \approx 1.41 \pm 0.88(1.74 \pm 0.81)$ using the condition of Equation (18). 
It is clearly seen from Figure 21 that below about $1.5 \rho_{0}$, the auxiliary-function-based (purple) and the conventional expansions (blue) give almost identical results. However, at higher densities, changing from Test Set I to Set III, the result from the auxiliary-functionbased approach is stable and always has smaller error bars compared to that from the conventional expansion. Moreover, the higher order contributions from $v_{\mathrm{sym}}^{3}$ are relatively small in the auxiliary-function-based reconstruction, indicating its fast convergence, by comparing Panel (c) with Panel (a) or Panel (b). As was pointed out in [60], this is because the function $v_{\text {sym }}$ itself generates higher order terms in $\chi$, e.g., $\chi^{3}, \chi^{4}$, etc., even when the symmetry energy is truncated apparently at order $v_{\text {sym }}^{2}$. Consequently, the reconstructed symmetry energy at suprasaturation densities from the auxiliary-function-based approach either to order $v_{\text {sym }}^{2}$ or to order $v_{\text {sym }}^{3}$ looks very similar.

Shown in Figure 22 are the reconstructed symmetry energy from $0.3 \rho_{0}$ to $3 \rho_{0}$ with the $1 \sigma$ uncertainty band using the exponential and algebraic auxiliary function, respectively, adopting Parameter Set III [60]. The $\Theta_{\text {sym }}$ parameter for the algebraic model was found to be about $\Theta_{\text {sym }} \approx 1.91 \pm 1.80$. Interestingly, the $E_{\text {sym }}(\rho)$ obtained from the two models (blue solid and black dashed) behaved very similarly, indicating the approximate independence of the auxiliary function used. Quantitatively, the symmetry energy at $2 \rho$ was found to be $E_{\text {sym }}^{\text {exp }}\left(2 \rho_{0}\right) \approx 44.8 \pm 8.1 \mathrm{MeV}$ and $E_{\text {sym }}^{\text {alge }}\left(2 \rho_{0}\right) \approx 46.4 \pm 9.1 \mathrm{MeV}$ with the two different auxiliary functions. They were in good agreement with the fiducial value of $E_{\text {sym }}\left(2 \rho_{0}\right) \approx$ $51 \pm 13 \mathrm{MeV}$ at a $68 \%$ confidence level from the nine different analyses of neutron star observables summarized in Figure 3 in Section 2.
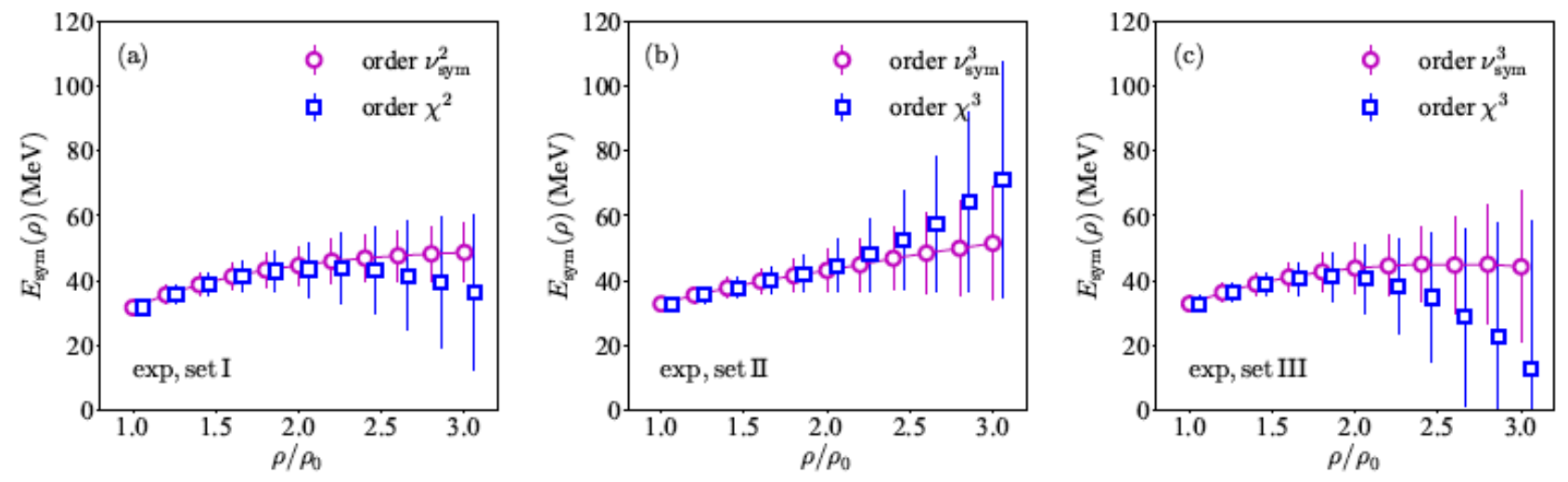

Figure 21. Density dependence of nuclear symmetry energy from simulations adopting the exponential model for the auxiliary function (purple) and the conventional Taylor expansion (blue) for Test Set I (a), II (b), and III (c), respectively. Taken from [60].

In short, knowing the characteristics of nuclear symmetry energy at $\rho_{0}$, regardless of how they were obtained, they can be used in properly chosen auxiliary functions to accurately predict the symmetry energy at suprasaturation densities up to about $3 \rho_{0}$ before the hadron-quark phase transition happens. Similar approaches can be developed to predict the SNM EOS or generally the EOS of isospin asymmetric nuclear matter at high densities using their characteristics at $\rho_{0}[60]$. 


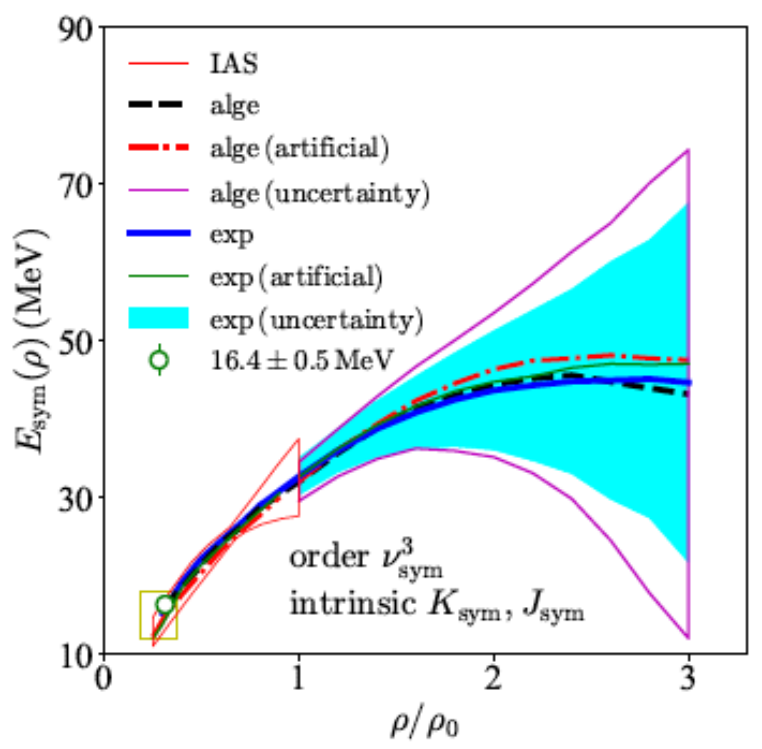

Figure 22. Symmetry energy reconstructed via the auxiliary functions in the exponential and algebraic models with Test Set III (adopting the intrinsic correlation between $K_{\text {sym }}$ and $J_{\text {sym }}$ imposed by the unbound nature of PNM). Taken from [60].

\section{Summary and Outlook}

The detection of the GW170817 event marked the beginning of gravitational wave astronomy. It stimulated many interesting new studies on the EOS of dense neutronrich matter. Together with observations of neutron stars using other messengers, as well as constraints from terrestrial nuclear experiments and predictions of nuclear theories, the tidal deformation of neutron stars from GW170817 has provided more information about the EOS of neutron star matter within various model frameworks. The nuclear symmetry energy encodes information about the energy cost to make nuclear matter more neutron rich, thus determining the content of neutron star matter. Many analyses of neutron star observables use models that directly construct/model/parameterize the pressure as a function of energy density above $\rho_{0}$ without considering explicitly the isospin degree of freedom, thus missing information about the symmetry energy at suprasaturation densities. To extract the latter, one has to model the EOS starting at the level of single-nucleon energy in neutron-rich matter with the explicit isospin degree of freedom. Indeed, many analyses have used such models. These analyses have extracted in various ways the characteristics of nuclear symmetry energy, especially its slope $\mathrm{L}$ and, to some extent, curvature $K_{\text {sym }}$ at $\rho_{0}$. Very few studies have tried to constrain the entire function of symmetry energy at high densities. Of course, there is the longstanding and interesting issue: At what density does the hadron-quark phase transition happen in neutron stars? An answer to this question is relevant for the study of high-density nuclear symmetry energy, as the latter will lose its original physical meaning once the phase transition happens. In turn, the high-density behavior of nuclear symmetry energy may affect the properties of the hadron-quark phase transition. Thus, ideally, the high-density symmetry energy should be extracted from neutron star observables using models encapsulating the hadron-quark phase transition and the explicit isospin degree of freedom for the hadronic phase.

In this brief review, within our limited knowledge range, we summarized what the community has extracted about the characteristics of nuclear symmetry energy at $\rho_{0}$ and some new understandings about its high-density behavior from analyzing neutron star observables since the discovery of GW170817. More specifically, corresponding to the questions listed in the introduction, in our possibly biased opinion, we learned the following: 
1. What have we learned about the symmetry energy from the tidal deformation of canonical neutron stars from GW170817, the mass of PSR J0740+6620, and NICER's simultaneous observation of the mass and radius of PSR J0030+0451 and PSR J0740+6620?

- $\quad$ The average value of the slope parameter $L$ of nuclear symmetry energy from 24 new analyses of neutron star observables was about $L \approx 57.7 \pm 19 \mathrm{MeV}$ at a $68 \%$ confidence level, while the average value of the curvature $K_{\text {sym }}$ from 16 new analyses was about $K_{\text {sym }} \approx-107 \pm 88 \mathrm{MeV}$, and the magnitude of nuclear symmetry energy at $2 \rho_{0}$ was found to be $E_{\text {sym }}\left(2 \rho_{0}\right) \approx 51 \pm 13 \mathrm{MeV}$ from nine new analyses;

- While the available data from canonical neutron stars did not provide tight constraints on nuclear symmetry energy at densities above about $2 \rho_{0}$, the lower radius boundary $R_{2.01}=12.2 \mathrm{~km}$ from NICER's very recent observation of PSR J0740+6620 having a mass of $2.08 \pm 0.07 M_{\odot}$ and radius $R=12.2-16.3 \mathrm{~km}$ at a $68 \%$ confidence level set a tighter lower limit for nuclear symmetry energy at densities above $2 \rho_{0}$ compared to what we knew before from analyzing earlier data;

2. How do the symmetry energy parameters extracted from recent observations of neutron stars compare with what we knew before the discovery of GW170817? Before GW170817, there were surveys of symmetry energy parameters based on over 50 analyses of various terrestrial nuclear experiments and some astrophysical observations of neutron stars. The newly extracted average value of $\mathrm{L}$ was in good agreement with the earlier fiducial value within the error bars. There was little information about the curvature $K_{\text {sym }}$ and $E_{\text {sym }}\left(2 \rho_{0}\right)$ before GW170817. The latter two quantities characterizing the symmetry energy from $\rho_{0} \sim 2 \rho_{0}$ were mostly from analyzing the new data of neutron star observations;

3. What can we learn about the high-density symmetry energy from future, more precise radius measurement of massive neutron stars? Using characteristically different mock mass-radius data up to $2 M_{\odot}$ within Bayesian analyses, it was found that the radius of massive neutron stars can constrain more tightly the lower boundary of high-density symmetry energy without much influence of the remaining uncertainties of SNM EOS. Indeed, as mentioned above, NICER's very recent observation of PSR J0740+6620 made this real. Moreover, the radii of massive neutron stars may help identify twin stars, the size of quark cores, and the nature of the hadron-quark phase transition [15,180,181];

4. What are the effects of hadron-quark phase transition on extracting the symmetry energy from neutron star observables? How does the symmetry energy affect the fraction and size of quark cores in hybrid stars? Bayesian inferences of nuclear symmetry energy using models encapsulating a first-order hadron-quark phase transition from observables of canonical neutron stars indicated that the phase transition shifts appreciably both $L$ and $K_{\text {sym }}$ to higher values, but with larger uncertainties compared to analyses assuming no such phase transition. It was also found that the available astrophysical data prefer the formation of a large volume of quark matter even in canonical NSs. The correlations among the symmetry energy parameters and the hadron-quark phase transition density, as well as the quark matter fraction were found to be weak. Moreover, the symmetry energy parameters extracted with or without considering the hadron-quark phase transition in neutron stars were all consistent with their known constraints within the still relatively large uncertainties. Thus, more precise constraints on the high-density symmetry energy are needed;

5. What are the effects of symmetry energy on the nature of GW190814's second component of mass (2.50-2.67) $M_{\odot}$ ? The high-density behavior of nuclear symmetry energy significantly affects the minimum rotational frequency of GW190814's secondary component of mass (2.50-2.67) $M_{\odot}$ as a superfast pulsar. It also affects the r-mode stability boundary of GW190814's secondary in the frequency-temperature plane. Moreover, its equatorial radius and Kepler frequency also depend strongly on the high-density behavior of nuclear symmetry energy; 
6. If all the characteristics of nuclear symmetry energy at saturation density $\rho_{0}$, e.g., its slope L, curvature $K_{\mathrm{sym}}$, and skewness $J_{\mathrm{sym}}$, are precisely determined by the astrophysical observations and/or terrestrial experiments, how do we use them to predict the symmetry energy at suprasaturation densities, such as $2-3 \rho_{0}$ ? It was found very recently that by expanding $E_{\text {sym }}(\rho)$ in terms of a properly chosen auxiliary function $\Pi_{\text {sym }}\left(\chi, \Theta_{\text {sym }}\right)$ with a parameter $\Theta_{\text {sym }}$ fixed accurately by an experimental $E_{\text {sym }}\left(\rho_{\mathrm{r}}\right)$ value at a reference density $\rho_{\mathrm{r}}$, the shortcomings of the conventional $\chi$-expansion can be completely removed or significantly reduced in determining the high-density behavior of $E_{\text {sym }}(\rho)$.

Thus, thanks to the historical GW170817 event and the hard work of many people in both astrophysics and nuclear physics, some interesting new knowledge about nuclear symmetry energy besides many other fundamental physics have been obtained from analyzing neutron star observables. Many interesting issues especially about the high-density behavior of nuclear symmetry energy remain to be resolved. More precise radius measurements of massive neutron stars are particularly useful for addressing these issues. Comprehensive analyses of combined multi-messengers from various astrophysical observatories, laboratory experiments, and nuclear theories will hopefully help us soon realize the ultimate goal of determining precisely the EOS of super-dense neutron-rich matter.

Author Contributions: Conceptualization, B.-A.L., B.-J.C., W.-J.X. and N.-B.Z.; methodology, B.-A.L., B.-J.C., W.-J.X. and N.-B.Z.; software, B.-A.L., B.-J.C., W.-J.X. and N.-B.Z.; validation, B.-A.L., B.J.C., W.-J.X. and N.-B.Z.; formal analysis, B.-A.L., B.-J.C., W.-J.X. and N.-B.Z.; investigation, B.-A.L., B.-J.C., W.-J.X. and N.-B.Z.; resources, B.-A.L., B.-J.C., W.-J.X. and N.-B.Z.; data curation, B.-A.L., B.-J.C., W.-J.X. and N.-B.Z.; writing-original draft preparation, B.-A.L., B.-J.C., W.-J.X. and N.-B.Z.; writing-review and editing, B.-A.L., W.-J.X. and N.-B.Z.; visualization, B.-A.L., B.-J.C., W.-J.X. and N.-B.Z.; supervision, B.-A.L.; project administration, B.-A.L.; funding acquisition, B.-A.L., B.-J.C., W.-J.X. and N.-B.Z. All authors have read and agreed to the published version of the manuscript.

Funding: This work was supported in part by the U.S. Department of Energy, Office of Science, under Award Number DE-SC0013702, the CUSTIPEN (China-U.S. Theory Institute for Physics with Exotic Nuclei) under the U.S. Department of Energy Grant No. DE-SC0009971, the Yuncheng University Research Project under Grant No. YQ-2017005, the National Natural Science Foundation of China under Grant No. 11505150 and No. 12005118, the Scientific and Technological Innovation Programs of Higher Education Institutions in Shanxi under Grant No. 2020L0550, and the Shandong Provincial Natural Science Foundation under Grant No. ZR2020QA085.

Institutional Review Board Statement: Not applicable.

Informed Consent Statement: Not applicable.

Data Availability Statement: The data presented in this study are available on request from the corresponding author.

Acknowledgments: We would like to thank Lie-Wen Chen, Wei-Zhou Jiang, Ang Li, De-Hua Wen, Jun $\mathrm{Xu}$, and $\mathrm{Xia} Z \mathrm{Zhou}$ for collaborations and discussion on some of the issues reviewed here.

Conflicts of Interest: The authors declare no conflict of interest.

\section{References}

1. The National Academies Press. New Worlds, New Horizons in Astronomy and Astrophysics; The National Academies Press: Washington, DC, USA, 2011.

Available online: https://www.nap.edu/catalog/12951/new-worlds-new-horizons-in-astronomy-and-astrophysics (accessed on 2 June 2021).

2. The National Academies Press. Nuclear Physics: Exploring the Heart of Matter; Report of the Committee on the Assessment of and Outlook for Nuclear Physics; The National Academies Press: Washington, DC, USA, 2012. Available online: https: / / www.nap.edu/catalog/13438/nuclear-physics-exploring-the-heart-of-matter (accessed on 2 June 2021).

3. 2015 U.S. Long Range Plan for Nuclear Sciences. Available online: https:/ / www.osti.gov/servlets/purl/1296778 (accessed on 2 June 2021).

4. The Nuclear Physics European Collaboration Committee (NuPECC). Long Range Plan 2017 Perspectives in Nuclear Physics. Available online: https:/ /www.esf.org/fileadmin/user_upload/esf/Nupecc-LRP2017.pdf (accessed on 2 June 2021). 
5. Lattimer, J.M.; Prakash, M. Neutron star structure and the equation of state. Astrophys. J. 2001, 550, 426-442. [CrossRef]

6. Lattimer, J.M.; Prakash, M. The equation of state of hot, dense matter and neutron stars. Phys. Rep. 2016, 621, 127-164. [CrossRef]

7. Watts, A.L.; Andersson, N.; Chakrabarty, D.; Feroci, M.; Hebeler, K.; Israel, G.; Lamb, F.K.; Miller, M.C.; Morsink, S.; Özel, F.; et al. Colloquium: Measuring the neutron star equation of state using X-ray timing. Rev. Mod. Phys. 2016, 88, 021001. [CrossRef]

8. Özel, F.; Freire, P. Masses, radii, and the equation of state of neutron stars. Annu. Rev. Astron. Astrophys. 2016, 88, 401-440. [CrossRef]

9. Oertel, M.; Hempel, M.; Klähn, T.; Typel, S. Equations of state for supernovae and compact stars. Rev. Mod. Phys. 2017, 89, 015007. [CrossRef]

10. Baiotti, L. Gravitational waves from neutron star mergers and their relation to the nuclear equation of state. Prog. Part. Nucl. Phys. 2019, 109, 103714. [CrossRef]

11. Li, B.A.; Krastev, P.G.; Wen, D.H.; Zhang, N.B. Towards understanding astrophysical effects of nuclear symmetry energy. Eur. Phys. J. A 2019, 55, 117. [CrossRef]

12. Weber, F.; Negreiros, R.; Roseneld, P.; Stejner, M. Pulsars as astrophysical laboratories for nuclear andparticle physics. Prog. Part. Nucl. Phys. 2007, 59, 94-113. [CrossRef]

13. Alford, M.G.; Han, S.; Schwenzer, K. Signatures for quark matter from multi-messenger observations. J. Phys. G Nucl. Part. Phys. 2019, 46, 114001. [CrossRef]

14. Capano, C.D.; Tews, I.; Brown, S.M.; Margalit, B.; De, S.; Kumar, S.; Brown, D.A.; Krishnan, B.; Reddy, S. Stringent constraints on neutron-star radii from multimessenger observations and nuclear theory. Nat. Astron. 2020, 4, 625-632. [CrossRef]

15. Blaschke, D.; Ayriyan, A.; Alvarez-Castillo, D.E.; Grigorian, H. Was GW170817 a canonical neutron star merger? Bayesian analysis with a third family of compact stars. Universe 2020, 6, 81. [CrossRef]

16. Chatziioannou, K. Neutron-star tidal deformability and equation-of-state constraints. Gen. Relativ. Gravit. 2020, 52, 109. [CrossRef]

17. Annala, E.; Gorda, T.; Kurkela, A.; Vuorinen, A. Gravitational-Wave Constraints on the Neutron-Star-Matter Equation of State. Phys. Rev. Lett. 2018, 120, 172703. [CrossRef] [PubMed]

18. Kievsky, A.; Viviani, M.; Logoteta, D.; Bombaci, I.; Girlanda, L. Correlations imposed by the unitary limit between few-nucleon systems and compact stellar systems. Phys. Rev. Lett. 2018, 121, 072901. [CrossRef]

19. Landry, P.; Essick, R.; Chatziioannou, K. Nonparametric constraints on neutron star matter with existing and upcoming gravitational wave and pulsar observations. Phys. Rev. D 2020, 101, 123007. [CrossRef]

20. Dietrich, T.; Coughlin, M.W.; Pang, P.T.H.; Bulla, M.; Heinzel, J.; Issa, L.; Tews, I.; Antier, S. Multimessenger constraints on the neutron-star equation of state and the Hubble constant. Science 2020, 370, 1450-1453. [CrossRef] [PubMed]

21. Li, A.; Zhu, Z.Y.; Zhou, E.P.; Dong, J.M.; Hu, J.N.; Xia, C.J. Neutron star equation of state: Quark mean-field (QMF) modeling and applications. J. High Energy Astrophys. 2020, 28, 19-46. [CrossRef]

22. Danielewicz, P.; Lacey, R.; Lynch, W.G. Determination of the equation of state of dense matter. Science 2002, $298,1592-1596$. [CrossRef]

23. Baran, V.; Colonna, M.; Greco, V.; Di Toro, M. Reaction dynamics with exotic nuclei. Phys. Rep. 2005, 410, 335-466. [CrossRef]

24. Steiner, A.W.; Prakash, M.; Lattimer, J.M.; Ellis, P.J. Isospin asymmetry in nuclei and neutron stars. Phys. Rep. 2005, 411, 325-375. [CrossRef]

25. Li, B.A.; Chen, L.W.; Ko, C.M. Recent Progress and New Challenges in Isospin Physics with Heavy-Ion Reactions. Phys. Rep. 2008, 464, 113-281. [CrossRef]

26. Chen, L.W. Higher order bulk characteristic parameters of asymmetric nuclear matter. Sci. China Phys. Mech. Astron. 2011, 54, 124-129. [CrossRef]

27. Trautmann, W.; Wolter, H.H. Elliptic flow and the symmetry energy at suprasaturation density. Int. J. Mod. Phys. E 2012, 21, 1230003. [CrossRef]

28. Tsang, M.B.; Stone, J.R.; Camera, F.; Danielewicz, P.; Gandolfi, S.; Hebeler, K.; Horowitz, C.J.; Lee, J.; Lynch, W.G.; Kohley, Z.; et al. Constraints on the symmetry energy and neutron skins from experiments and theory. Phys. Rev. C 2012, 86, 015803. [CrossRef]

29. Li, B.A.; Ramos, À.; Verde, G.; Vidaña, I. Topical Issue on Nuclear Symmetry Energy. Eur. Phys. J. A 2014, 50, 9. [CrossRef]

30. Baldo, M.; Burgio, G.F. The nuclear symmetry energy. Prog. Part. Nucl. Phys. 2016, 91, 203-258. [CrossRef]

31. Li, B.A. Nuclear symmetry energy extracted from laboratory experiments. Nucl. Phys. News 2017, 27, 7-11. [CrossRef]

32. Li, B.A.; Cai, B.J.; Chen, L.W.; Xu, J. Nucleon effective masses in neutron-rich matter. Prog. Part. Nucl. Phys. 2018, 99, 29-119. [CrossRef]

33. Burgio, G.F.; Vidaña, I. The Equation of State of Nuclear Matter: From Finite Nuclei to Neutron Stars. Universe 2020, 6, 119. [CrossRef]

34. Bombaci, I.; Lombardo, U. Asymmetric nuclear matter equation of state. Phys. Rev. C 1991, 44, 1892. [CrossRef]

35. Li, B.A.; Xie, W.J. Symmetry energy of super-dense neutron-rich matter from integrating barotropic pressures in neutron stars and heavy-ion reactions. Phys. Lett. B 2020, 806, 135517. [CrossRef]

36. Newton, W.G.; Li, B.A. Constraining the gravitational binding energy of PSR J0737-3039B using terrestrial nuclear data. Phys. Rev. C 2009, 80, 065809. [CrossRef]

37. He, X.T.; Fattoyev, F.J.; Li, B.A.; Newton, W.G. Impact of the equation-of-state-gravity degeneracy on constraining the nuclear symmetry energy from astrophysical observables. Phys. Rev. C 2015, 91, 015810. [CrossRef] 
38. Psaltis, D. Probes and Tests of Strong-Field Gravity with Observations in the Electromagnetic Spectrum. Living Rev. Relativ. 2008, 11, 9. [CrossRef] [PubMed]

39. Yunes, N.; Psaltis, D.; Özel, F.; Loeb, A. Constraining Parity Violation in Gravity with Measurements of Neutron-Star Moments of Inertia. Phys. Rev. D 2010, 81, 064020. [CrossRef]

40. DeDeo, S.; Psaltis, D. Towards New Tests of Strong-field Gravity with Measurements of Surface Atomic Line Redshifts from Neutron Stars. Phys. Rev. Lett. 2003, 90, 141101. [CrossRef]

41. Wen, D.-H.; Li, B.-A.; Chen, L.-W. Can the maximum mass of neutron stars rule out any equation of state of dense stellar matter before gravity is well understood? arXiv 2011, arXiv:1101.1504.

42. Zhang, N.B.; Li, B.A. GW190814's Secondary Component with Mass 2.50-2.67 $M_{\odot}$ as a Superfast Pulsar. Astrophys. J. 2020, 902, 38. [CrossRef]

43. Zhou, X.; Li, A.; Li, B.A. R-mode Stability of GW190814's Secondary Component as a Supermassive and Superfast Pulsar. Astrophys. J. 2021, 910, 62. [CrossRef]

44. Drago, A.; Lavagno, A.; Pagliara, G.; Pigato, D. Early appearance of $\Delta$ isobars in neutron stars. Phys. Rev. C 2014, 90, 065809. [CrossRef]

45. Cai, B.J.; Fattoyev, F.J.; Li, B.A.; Newton, W.G. Critical density and impact of $\Delta(1232)$ resonance formation in neutron stars. Phys. Rev. C 2015, 92, 015802. [CrossRef]

46. Zhu, Z.Y.; Li, A.; Hu, J.N.; Sagawa, H. $\Delta$ (1232) effects in density-dependent relativistic hartree-fock theory and neutron stars. Phys. Rev. C 2016, 94, 045803. [CrossRef]

47. Sahoo, H.S.; Mitra, G.; Mishra, R.; Panda, P.K.; Li, B.A. Neutron star matter with $\Delta$ isobars in a relativistic quark model. Phys. Rev. C 2018, 98, 045801. [CrossRef]

48. Li, J.J.; Sedrakian, A.; Weber, F. Competition between delta isobars and hyperons and properties of compact stars. Phys. Lett. B 2018, 783, 234-240. [CrossRef]

49. Ribes, P.; Ramos, A.; Tolos, L.; Gonzalez-Boquera, C.; Centelles, M. Interplay between $\Delta$ Particles and Hyperons in Neutron Stars. Astrophys. J. 2019, 883, 168. [CrossRef]

50. Li, J.J.; Sedrakian, A. Implications from GW170817 for Delta-isobar Admixed Hypernuclear Compact Stars. Astrophys. J. Lett. 2019, 874, L22. [CrossRef]

51. Raduta, A.R.; Oertel, M.; Sedrakian, A. Proto-neutron stars with heavy baryons and universal relations. Mon. Not. R. Astron. Soc. 2020, 499, 914-931. [CrossRef]

52. Raduta, A.R. $\Delta$-admixed neutron stars: Spinodal instabilities and dUrca processes. Phys. Lett. B 2021, 814, 136070. [CrossRef]

53. Thapa, V.B.; Sinha, M.; Li, J.J.; Sedrakian, A. Massive $\Delta$-resonance admixed hypernuclear stars with antikaon condensations. Phys. Rev. D 2021, 103, 063004. [CrossRef]

54. Sen, D. Variation of the $\Delta$ baryon mass and hybrid star properties in static and rotating conditions. Phys. Rev. C 2021, 103, 045804. [CrossRef]

55. Jiang, W.Z.; Li, B.A.; Chen, L.W. Large-mass neutron stars with hyperonization. Astrophys. J. 2012, 756, 56. [CrossRef]

56. Providência, C.; Fortin, M.; Pais, H.; Rabhi, A. Hyperonic stars and the nuclear symmetry energy. Front. Astron. Space Sci. 2019, 6, 13. [CrossRef]

57. Vidaña, I. Hyperons: The strange ingredients of the nuclear equation of state. Proc. R. Soc. Lond. A 2018, 474, 20180145. [CrossRef]

58. Choi, S.; Miyatsu, T.; Cheoun, M.K.; Saito, K. Constraints on Nuclear Saturation Properties from Terrestrial Experiments and Astrophysical Observations of Neutron Stars. Astrophys. J. 2021, 909, 156. [CrossRef]

59. Fortin, M.; Raduta, A.R.; Avancini, S.; Providência, C. Thermal evolution of relativistic hyperonic compact stars with calibrated equations of state. Phys. Rev. D 2021, 103, 083004. [CrossRef]

60. Cai, B.J.; Li, B.A. Auxiliary Function Approach for Determining Symmetry Energy at Supra-saturation Densities. Phys. Rev. C 2021, 103, 054611. [CrossRef]

61. Li, B.A.; Han, X. Constraining the neutron-proton effective mass splitting using empirical constraints on the density dependence of nuclear symmetry energy around normal density. Phys. Lett. B 2013, 727, 276-281. [CrossRef]

62. Drischler, C.; Furnstahl, R.J.; Melendez, J.A.; Phillips, D.R. How well do we know the neutron-matter equation of state at the densities inside neutron stars? A Bayesian approach with correlated uncertainties. Phys. Rev. Lett. 2020, 125, 202702. [CrossRef]

63. Mondal, C.; Agrawal, B.K.; De, J.N.; Samaddar, S.K.; Centelles, M.; Viñas, X. Interdependence of different symmetry energy elements. Phys. Rev. C 2017, 96, 021302. [CrossRef]

64. Malik, T.; Alam, N.; Fortin, M.; Providência, C.; Agrawal, B.K.; Jha, T.K.; Kumar, B.; Patra, S.K. GW170817: Constraining the nuclear matter equation of state from the neutron star tidal deformability. Phys. Rev. C 2018, 98, 035804. [CrossRef]

65. Biswas, B.; Char, P.; Nandi, R.; Bose, S. Towards mitigation of apparent tension between nuclear physics and astrophysical observations by improved modeling of neutron star matter. arXiv 2020, arXiv:2008.01582.

66. Tsang, C.Y.; Tsang, M.B.; Danielewicz, P.; Lynch, W.G.; Fattoyev, F.J. Impact of the neutron-star deformability on equation of state parameters. Phys. Rev. C 2020, 102, 045808. [CrossRef]

67. Xie, W.J.; Li, B.A. Bayesian Inference of the Symmetry Energy of Superdense Neutron-rich Matter from Future Radius Measurements of Massive Neutron Stars. Astrophys. J. 2020, 899, 4. [CrossRef]

68. d'Etivaux, N.B.; Guillot, S.; Margueron, J.; Webb, N.; Catelan, M.; Reisenegger, A. New constraints on the nuclear equation of state from the thermal emission of neutron stars in quiescent low-mass X-ray binaries. Astrophys. J. 2019, 887, 48. [CrossRef] 
69. Malik, T.; Agrawal, B.K.; Providência, C.; De, J.N. Unveiling the correlations of tidal deformability with the nuclear symmetry energy parameters. Phys. Rev. C 2020, 102, 052801. [CrossRef]

70. Zhao, T.; Lattimer, J.M. Tidal Deformabilities and Neutron Star Mergers. Phys. Rev. D 2018, 98, 063020. [CrossRef]

71. Lim, Y.; Holt, J.W. Bayesian modeling of the nuclear equation of state for neutron star tidal deformabilities and GW170817. Eur. Phys. J. A 2019, 55, 209. [CrossRef]

72. Margueron, J.; Casali, R.H.; Gulminelli, F. Equation of state for dense nucleonic matter from metamodeling. II. Predictions for neutron star properties. Phys. Rev. C 2018, 97, 025806. [CrossRef]

73. Tan, N.H.; Khoa, D.T.; Loan, D.T. Spin-polarized $\beta$-stable neutron star matter: The nuclear symmetry energy and GW170817 constraint. Phys. Rev. C 2020, 102, 045809. [CrossRef]

74. Zhang, Y.; Liu, M.; Xia, C.J.; Li, Z.; Biswal, S.K. Constraints on the symmetry energy and its associated parameters from nuclei to neutron stars. Phys. Rev. C 2020, 101, 034303. [CrossRef]

75. Chamel, N.; Pearson, J.M.; Potekhin, A.Y.; Fantina, A.F.; Ducoin, C.; Dutta, A.; Goriely, S.; Perot, L. Role of the Symmetry Energy on the Structure of Neutron Stars with Unified Equations of State. AIP Conf. Proc. 2019, 2127, 020021. [CrossRef]

76. Huang, K.X.; Hu, J.N.; Zhang, Y.; Shen, H. The possibility of the secondary object in GW190814 as a neutron star. Astrophys. J. 2020, 904, 39. [CrossRef]

77. Tews, I.; Margueron, J.; Reddy, S. Confronting gravitational-wave observations with modern nuclear physics constraints. Eur. Phys. J. A 2019, 55, 97. [CrossRef]

78. Gil, H.; Kim, Y.M.; Papakonstantinou, P.; Ho, C. Constraining the density dependence of the symmetry energy with nuclear data and astronomical observations in the KIDS framework. Phys. Rev. C 2021, 103, 034330. [CrossRef]

79. Raithel, C.A.; Özel, F. Measurement of the nuclear symmetry energy parameters from gravitational wave events. Astrophys. J. 2019, 885, 121. [CrossRef]

80. Yue, T.G.; Chen, L.W.; Zhang, Z.; Zhou, Y. Constraints on the Symmetry Energy from PREX-II in the Multimessenger Era. arXiv 2021, arXiv:2102.05267.

81. Essick, R.; Tews, I.; Landry, P.; Schwenk, A. Astrophysical Constraints on the Symmetry Energy and the Neutron Skin of ${ }^{208} \mathrm{~Pb}$ with Minimal Modeling Assumptions. arXiv 2021, arXiv:2102.10074.

82. Li, B.A.; Magno, M. Curvature-slope correlation of nuclear symmetry energy and its imprints on the crust-core transition, radius and tidal deformability of canonical neutron stars. Phys. Rev. C 2020, 102, 045807. [CrossRef]

83. Estee, J.; et al. [S $\pi$ RIT Collaboration]. Probing the Symmetry Energy with the Spectral Pion Ratio. Phys. Rev. Lett. 2021, 126, 162701. [CrossRef]

84. Adhikari, D.; et al. [PREX Collaboration]. An Accurate Determination of the Neutron Skin Thickness of ${ }^{208} \mathrm{~Pb}$ through ParityViolation in Electron Scattering. Phys. Rev. Lett. 2021, 126, 172502. [CrossRef]

85. Reed, B.T.; Fattoyev, F.J.; Horowitz, C.J.; Piekarewicz, J. Implications of PREX-II on the equation of state of neutron-rich matter. Phys. Rev. Lett. 2021, 126, 172503. [CrossRef]

86. Xu, J.; Xie, W.J.; Li, B.A. Bayesian inference of nuclear symmetry energy from measured and imagined neutron skin thickness in 116,118,120,122,124,130,132 Sn, ${ }^{208} \mathrm{~Pb}$, and ${ }^{48} \mathrm{Ca}$. Phys. Rev. C 2020, 102, 044316. [CrossRef]

87. Biswas, B. Impact of PREX-II, the revised mass measurement of PSRJ0740+6620, and possible NICER observation on the dense matter equation of state. arXiv 2021, arXiv:2105.02886.

88. Zimmerman, J.; Carson, Z.; Schumacher, K.; Steiner, A.W.; Yagi, K. Measuring Nuclear Matter Parameters with NICER and LIGO/Virgo. arXiv 2020, arXiv:2002.03210.

89. Carson, Z.; Steiner, A.W.; Yagi, K. Future Prospects for Constraining Nuclear Matter Parameters with Gravitational Waves. Phys. Rev. D 2019, 100, 023012. [CrossRef]

90. Tews, I.; Lattimer, J.M.; Ohnishi A.; Kolomeitsev, E.E. Symmetry Parameter Constraints from a Lower Bound on Neutron-matter Energy. Astrophys. J. 2017, 848, 105. [CrossRef]

91. Zhang, N.B.; Cai, B.J.; Li, B.A.; Newton W.G.; Xu, J. How tightly is the nuclear symmetry energy constrained by a unitary Fermi gas? Nucl. Sci. Tech. 2017, 28, 181. [CrossRef]

92. Holt J.; Lim, Y. Universal correlations in the nuclear symmetry energy, slope parameter, and curvature. Phys. Lett. B 2018, $784,77$. [CrossRef]

93. Russotto, P.; Wu, P.Z.; Zoric, M.; Chartier, M.; Leifels, Y.; Lemmon, R.C.; Li, Q.; Łukasik, J.; Pagano, A.; Pawłowski, P.; et al. Symmetry energy from elliptic flow in ${ }^{197} \mathrm{Au}+{ }^{197} \mathrm{Au}$. Phys. Lett. B 2011, 697, 471-476. [CrossRef]

94. Russotto, P.; Gannon, S.; Kupny, S.; Lasko, P.; Acosta, L.; Adamczyk, M.; Al-Ajlan, A.; Al-Garawi, M.; Al-Homaidhi, S.; Amorini, F.; et al. Results of the ASY-EOS experiment at GSI: The symmetry energy at suprasaturation density. Phys. Rev. C 2016, 94, 034608. [CrossRef]

95. Zhang, N.B.; Li, B.A.; Xu, J. Combined Constraints on the Equation of State of Dense Neutron-rich Matter from Terrestrial Nuclear Experiments and Observations of Neutron Stars. Astrophys. J. 2018, 859, 90. [CrossRef]

96. Zhang, N.B.; Li, B.A. Extracting Nuclear Symmetry Energies at High Densities from Observations of Neutron Stars and Gravitational Waves. Eur. Phys. J. A 2019, 55, 39. [CrossRef]

97. Zhang, N.B.; Li, B.A. Implications of the Mass $M=2.17_{-0.10}^{+0.11} M_{\odot}$ of PSR J0740+6620 on the Equation of State of Super-dense Neutron-rich Nuclear Matter. Astrophys. J. 2019, 879, 99. [CrossRef] 
98. Xie, W.J.; Li, B.A. Bayesian Inference of High-density Nuclear Symmetry Energy from Radii of Canonical Neutron Stars. Astrophys. J. 2019, 883, 174. [CrossRef]

99. Zhou, Y.; Chen, L.W.; Zhang, Z. Equation of state of dense matter in the multimessenger era. Phys. Rev. D 2019, 99, 121301. [CrossRef]

100. Nakazato, K.; Suzuki, H. Cooling timescale for protoneutron stars and properties of nuclear matter: Effective mass and symmetry energy at high densities. Astrophys. J. 2019, 878, 25. [CrossRef]

101. Lonardoni, D.; Tews, I.; Gandolfi, S.; Carlson, J. Nuclear and neutron-star matter from local chiral interactions. Phys. Rev. Res. 2020, 2, 022033(R). [CrossRef]

102. Zhang, N.B.; Li, B.A. Delineating Effects of Nuclear Symmetry Energy on the Radii and Tidal Polarizabilities of Neutron Stars. J. Phys. G 2019, 46, 014002. [CrossRef]

103. Abbott, B.P.; et al. [The LIGO Scientific Collaboration and the Virgo Collaboration]. GW170817: Measurements of neutron star radii and equation of state. Phys. Rev. Lett. 2018, 121, 161101. [CrossRef]

104. Miller, M.C.; Lamb, F.K.; Dittmann, A.J.; Bogdanov, S.; Arzoumanian, Z.; Gendreau, K.C.; Guillot, S.; Harding, A.K.; Ho, W.C.G.; Lattimer, J.M.; et al. PSR J0030+0451 mass and radius from NICER data and implications for the properties of neutron star matter. Astrophys. J. Lett. 2019, 887, L24. [CrossRef]

105. Lattimer, J.M.; Steiner, A.W. Constraints on the symmetry energy using the mass-radius relation of neutron stars. Eur. Phys. J. A 2014, 50, 40. [CrossRef]

106. Cromartie, H.T.; Fonseca, E.; Ransom, S.M.; Demorest, P.B.; Arzoumanian, Z.; Blumer, H.; Brook, P.R.; DeCesar, M.E.; Dolch, T.; Ellis, J.A.; et al. Relativistic Shapiro delay measurements of an extremely massive millisecond pulsar. Nat. Astron. 2020, 4, 72-76. [CrossRef]

107. Fonseca, E.; Cromartie, H.; Pennucci, T.T.; Ray, P.S.; Kirichenko, A.Y.; Ransom, S.M.; Demorest, P.B.; Stairs, I.H.; Arzoumanian, Z.; Guillemot, L.; et al. Refined Mass and Geometric Measurements of the High-Mass PSR J0740+6620. arXiv 2021, arXiv:2104.00880.

108. Miller, M.C.; Lamb, F.K.; Dittmann, A.J.; Bogdanov, S.; Arzoumanian, Z.; Gendreau, K.C.; Guillot, S.; Ho, W.C.G.; Lattimer, J.M.; Loewenstein, M.; et al. The Radius of PSR J0740+6620 from NICER and XMM-Newton Data. arXiv 2021, arXiv:2105.06979.

109. Riley, T.E.; Watts, A.L.; Ray, P.S.; Bogdanov, S.; Guillot, S.; Morsink, S.M.; Bilous, A.V.; Arzoumanian, Z.; Choudhury, D.; Deneva, J.S.; et al. A NICER View of the Massive Pulsar PSR J0740+6620 Informed by Radio Timing and XMM-Newton Spectroscopy. arXiv 2021, arXiv:2105.06980.

110. Zhang, N.B.; Li, B.A. Constraints on the muon fraction and density profile in neutron stars. Astrophys. J. 2020, 893, 61. [CrossRef]

111. Zhou, Y.; Chen, L.W. Ruling out the supersoft high-density symmetry energy from the discovery of PSR J0740+6620 with mass $2.14_{-0.09}^{+0.10} M_{\odot}$. Astrophys. J. 2019, 886, 52. [CrossRef]

112. Riley, T.E.; Watts, A.L.; Bogdanov, S.; Ray, P.S.; Ludlam, R.M.; Guillot, S.; Arzoumanian, Z.; Baker, C.L.; Bilous, A.V.; Chakrabarty, D. A NICER View of PSR J0030+0451: Millisecond Pulsar Parameter Estimation.

Astrophys. J. Lett. 2019, 887, L21. [CrossRef]

113. Miller, M.C. NICER Measurements of Pulsars PSR J0030+0451 and PSR J0740+6620. Seminar at the Institute for Nuclear Theory, University of Washington, 22 April 2021. Available online: https://www.int.washington.edu/talks/S@INTtalks/Miller_Apr_22 2021.pdf (accessed on 2 June 2021).

114. Zhang, N.B.; Li, B.A. Impacts of NICER's Radius Measurement of PSR J0740+6620 on Nuclear Symmetry Energy at Suprasaturation Densities. arXiv 2021, arXiv:2105.11031.

115. Chen, L.W. Symmetry energy systematics and its high density behavior. Eur. Phys. J. Web Conf. 2018, 88, 00017. [CrossRef]

116. De, S.; Finstad, D.; Lattimer, J.M.; Brown, D.A.; Berger, E.; Biwer, C.M. Tidal deformabilities and radii of neutron stars from the observation of GW170817. Phys. Rev. Lett. 2018, 121, 091102. [CrossRef]

117. Fortin, M.; Providência, C.; Raduta, Ad.R.; Gulminelli, F.; Zdunik, J.L.; Haensel, P.; Bejger, M. Neutron star radii and crusts: Uncertainties and unified equations of state. Phys. Rev. C 2016, 94, 035804. [CrossRef]

118. Newton, W.G.; Crocombe, G. The nuclear symmetry energy from neutron skins and pure neutron matter in a Bayesian framework. arXiv 2020, arXiv:2008.00042.

119. Alford, M.; Braby, M.; Paris, M.; Reddy, S. Hybrid stars that masquerade as neutron stars. Astrophys. J. 2005, 629, 969-978. [CrossRef]

120. Bauswein, A.; Bastian, N.U.F.; Blaschke, D.B.; Chatziioannou, K.; Clark, J.A.; Fischer, T.; Oertel, M. Identifying a first-order phase transition in neutron star mergers through gravitational waves. Phys. Rev. Lett. 2019, 122, 061102. [CrossRef] [PubMed]

121. Weih, L.R.; Hanauske, M.; Rezzolla, L. Postmerger Gravitational-Wave Signatures of Phase Transitions in Binary Mergers. Phys. Rev. Lett. 2020, 124, 171103. [CrossRef] [PubMed]

122. Xie, W.J.; Li, B.A. Bayesian inference of the dense-matter equation of state encapsulating a first-order hadron-quark phase transition from observables of canonical neutron stars. Phys. Rev. C 2021, 103, 035802. [CrossRef]

123. Alford, M.G.; Han, S.; Prakash, M. Generic conditions for stable hybrid stars. Phys. Rev. D 2013, 88, 083013. [CrossRef]

124. Tang, S.P.; Jiang, J.L.; Gao, W.H.; Fan, Y.Z.; Wei, D.M. Constraint on phase transition with the multimessenger data of neutron stars. Phys. Rev. D 2021, 103, 063026. [CrossRef]

125. Li, A.; Miao, Z.; Han, S.; Zhang, B. Constraints on the maximum mass of neutron stars with a quark core from GW170817 and NICER PSR J0030+0451 data. Astrophys. J. 2021, 913, 27. [CrossRef] 
126. Annala, E.; Gorda, T.; Kurkela, A.; Nättilä, J.; Vuorinen, A. Evidence for quark-matter cores in massive neutron stars. Nat. Phys. 2020, 16, 907-910. [CrossRef]

127. Abbott, R.; et al. [LIGO Scientific Collaboration and Virgo Collaboration]. GW190814: Gravitational Waves from the Coalescence of a 23 Solar Mass Black Hole with a 2.6 Solar Mass Compact Object. Astrophys. J. Lett. 2020, 896, L44. [CrossRef]

128. Farr, W.M.; Sravan, N.; Cantrell, A.; Kreidberg, L.; Bailyn, C.D. The mass distribution of stellar-mass balck holes. Astrophys. J. 2011, 741, 103. [CrossRef]

129. Özel, F.; Psaltis, D.; Narayan, R.; McClintock, J.E. The black hole mass distribution in the galaxy. Astrophys. J. 2010, $725,1918$. [CrossRef]

130. Farr, W.M.; Chatziioannou, K. A Population-Informed Mass Estimate for Pulsar J0740+6620. Res. Notes AAS 2020, 4, 65. [CrossRef]

131. Abbott, B.P.; et al. [LIGO Scientific Collaboration and Virgo Collaboration]. GW170817: Observation of Gravitational Waves from a Binary Neutron Star Inspiral. Phys. Rev. Lett. 2017, 119, 161101. [CrossRef]

132. Safarzadeh M.; Loeb, A. Formation of mass gap objects in highly asymmetric mergers. Astrophys. J. Lett. 2020, 899, L15. [CrossRef]

133. Vattis, K.; Goldstein, I.S.; Koushiappas, S.M. Could the $2.6 M_{\odot}$ object in GW190814 be a primordial black hole? Phys. Rev. D 2020, 102, 061301. [CrossRef]

134. Zevin, M.; Spera, M.; Berry, C.P.; Kalogera, V. Exploring the Lower Mass Gap and Unequal Mass Regime in Compact Binary Evolution. Astrophys. J. Lett. 2020, 899, L1. [CrossRef]

135. Essick, R.; Landry, P. Discriminating between Neutron Stars and Black Holes with Imperfect Knowledge of the Maximum Neutron Star Mass. Astrophys. J. 2020, 904, 80. [CrossRef]

136. Tews, I.; Pang, P.T.H.; Dietrich, T.; Coughlin, M.W.; Antier, S.; Bulla, M.; Heinzel, J.; Issa, L. On the nature of GW190814 and its impact on the understanding of supranuclear matter. Astrophys. J. Lett. 2021, 908, L1. [CrossRef]

137. Fattoyev, F.J.; Horowitz, C.J.; Piekarewicz, J.; Reed, B. GW190814: Impact of a 2.6 solar mass neutron star on the nucleonic equations of state. Phys. Rev. C 2020, 102, 065805. [CrossRef]

138. Das, H.C.; Kumar, A.; Kumar, B.; Biswal, S.K.; Patra, S.K. The BigApple force and it's implications to finite nuclei and astrophysical objects. arXiv 2020, arXiv:2009.10690.

139. Li, J.J.; Sedrakian, A.; Weber, F. Rapidly rotating $\Delta$-resonance-admixed hypernuclear compact stars. Phys. Lett. B 2020, 810, 135812. [CrossRef]

140. Sedrakian, A.; Weber, F.; Li, J.J. Confronting GW190814 with hyperonization in dense matter and hypernuclear compact stars. Phys. Rev. D 2020, 102, 041301(R). [CrossRef]

141. Biswal, S.K.; Das, H.C.; Kumar, A.; Kumar, B.; Patra, S.K. Correlation between the curvature and some properties of the neutron star. arXiv 2020, arXiv:2012.13673.

142. Bombaci, I.; Drago, A.; Logoteta, D.; Pagliara, G.; Vidaña, I. Was GW190814 a black hole—Strange quark star system? Phys. Rev. Lett. 2021, 126, 162702. [CrossRef]

143. Godzieba, D.A.; Radice, D.; Bernuzzi, S. On the Maximum Mass of Neutron Stars and GW190814. Astrophys. J. 2021, 908, 122. [CrossRef]

144. Gonçalves, V.P.; Lazzari, L. Electrically charged strange stars with an interacting quark matter equation of state. Phys. Rev. D 2020, 102, 034031. [CrossRef]

145. Lim, Y.; Bhattacharya, A.; Holt, J.W.; Pati, D. Radius and equation of state constraints from massive neutron stars and GW190814. arXiv 2021, arXiv:2007.06526.

146. Lourenço, O.; Lenzi, C.H.; Dutra, M. Tidal deformability of strange stars and the GW170817 event. Phys. Rev. D 2021, 103, 103010. [CrossRef]

147. Rather, I.A.; Rahaman, U.; Dexheimer, V.; Usmani, A.A.; Patra, S.K. Heavy Magnetic Neutron Stars. arXiv 2021, arXiv:2104.05950.

148. Roupas, Z.; Panotopoulos, G.; Lopes, I. QCD color superconductivity in compact stars: Color-flavor locked quark star candidate for the gravitational-wave signal GW190814. arXiv 2020, arXiv:2010.11020.

149. Tan, H.; Noronha-Hostler, J.; Yunes, N. Neutron Star Equation of State in Light of GW190814. Phys. Rev. Lett. 2020, 125, 261104. [CrossRef]

150. Wu, X.H.; Bao, S.S.; Shen, H.; Xu, R.X. Symmetry energy effect on the secondary component of GW190814 as a neutron star. arXiv 2021, arXiv:2101.05476.

151. Demircik, T.; Ecker, C.; Järvinen, M. Rapidly Spinning Compact Stars with Deconfinement Phase Transition. Astrophys. J. Lett. 2021, 907, L37. [CrossRef]

152. Dexheimer, V.; Gomes, R.O.; Klähn, T.; Han, S.; Salinas, M. GW190814 as a massive rapidly rotating neutron star with exotic degrees of freedom. Phys. Rev. C 2021, 103, 0025808. [CrossRef]

153. Khadkikar, S.; Raduta, A.R.; Oertel, M.; Sedrakian, A. Maximum mass of compact stars from gravitational wave events with finite-temperature equations of state. arXiv 2021, arXiv:2102.00988.

154. Most, E.R.; Papenfort, L.J.; Weih, L.R.; Rezzolla, L. A lower bound on the maximum mass if the secondary in GW190814 was once a rapidly spinning neutron star. Mon. Not. R. Astron. Soc. Lett. 2020, 499, L82. [CrossRef]

155. Riahi, R.; Kalantari, S.Z. Properties of Rotating Neutron Star in Density-dependent Relativistic Mean-field Models. Int. J. Mod. Phys. D 2021, 30, 2150001. [CrossRef]

156. Stone, J.R.; Dexheimer, V.; Guichon, P.A.M.; Thomas, A.W.; Typel, S. Equation of state of hot dense hyperonic matter in the Quark-Meson-Coupling (QMC-A) model. Mon. Not. R. Astron. Soc. 2021, 502, 3476-3490. [CrossRef] 
157. Tsokaros, A.; Ruiz, M.; Shapiro, S.L. GW190814: Spin and Equation of State of a Neutron Star Companion. Astrophys. J. 2020, 905, 48. [CrossRef]

158. Christian, J.E.; Schaffner-Bielich, J. Supermassive neutron stars rule out twin stars. Phys. Rev. D 2020, 103, 063042. [CrossRef]

159. Thapa, V.B.; Sinha, M. Dense matter equation of state of a massive neutron star with antikaon condensation. Phys. Rev. D 2020, 102, 123007. [CrossRef]

160. Hessels, J.W.; Ransom, S.M.; Stairs, I.H.; Freire, P.C.; Kaspi, V.M.; Camilo, F. A radio pulsar spinning at 716 Hz. Science 2006, 311, 1901-1904. [CrossRef] [PubMed]

161. Biswas, B.; Nandi, R.; Char, P.; Bose, S.; Stergioulas, N. GW190814: On the properties of the secondary component of the binary. arXiv 2020, arXiv:2010.02090.

162. Andersson, N.; Kokkotas, K.D. The r-mode instability in rotating neutron stars. Int. J. Mod. Phys. D 2001, 10, 381-441. [CrossRef]

163. Chandrasekhar, S. Solutions of two problems in the theory of gravitational radiation. Phys. Rev. Lett. 1970, 24, 611. [CrossRef]

164. Friedman, J.L.; Schutz, B.F. Secular instability of rotating Newtonian stars. Astrophys. J. 1978, 222, 281. [CrossRef]

165. Krastev, P.G.; Li, B.A.; Worley, A. Constraining properties of rapidly rotating neutron stars using data from heavy-ion collisions. Astrophys. J. 2008, 676, 1170-1177. [CrossRef]

166. Worley, A.; Krastev, P.G.; Li, B.A. Nuclear constraints on the momenta of inertia of neutron stars. Astrophys. J. 2008, 685, 390-399. [CrossRef]

167. Wen, D.H.; Newton, W.G.; Li, B.A. Sensitivity of the neutron star -mode instability window to the density dependence of the nuclear symmetry energy. Phys. Rev. C 2012, 85, 025801. [CrossRef]

168. Vidaña, I. Nuclear symmetry energy and the r-mode instability of neutron stars. Phys. Rev. C 2012, 85, 045808; Erratum in Phys. Rev. C 2014, 90, 029901. [CrossRef]

169. Stergioulas, N. RNS Code. 1995. Available online: http:/ / www.gravity.phys.uwm.edu/rns/ (accessed on 2 June 2021).

170. Danielewicz, P.; Lee, J. Symmetry Energy II: Isobaric Analog States. Nucl. Phys. A 2014, 922, 1-70. [CrossRef]

171. Zhang, Z.; Chen, L.W. Electric dipole polarizability in ${ }^{208} \mathrm{~Pb}$ as a probe of the symmetry energy and neutron matter around $\rho_{0} / 3$. Phys. Rev. C 2015, 92, 031301. [CrossRef]

172. Xu, J.; Zhou, J.; Zhang, Z.; Xie, W.J.; Li, B.A. Constraining isovector nuclear interactions with giant resonances within a Bayesian approach. Phys. Lett. B 2020, 810, 135820. [CrossRef]

173. Cai, B.J.; Li, B.A. Intrinsic correlations among characteristics of neutron-rich matter imposed by the unbound nature of pure neutron matter. Phys. Rev. C 2021, 103, 034607. [CrossRef]

174. Garg, U.; Colò, G. The compression-mode giant resonances and nuclear incompressibility. Prog. Part. Nucl. Phys. 2018, 101, 55-95. [CrossRef]

175. Youngblood, D.H.; Clark, H.L.; Lui, Y.W. Incompressibility of Nuclear Matter from the Giant Monopole Resonance. Phys. Rev. Lett. 1999, 82, 691. [CrossRef]

176. Shlomo, S.; Kolomietz, V.M.; Colo, G. Deducing the nuclear-matter incompressibility coefficient from data on isoscalar compression modes. Eur. Phys. J. A 2006, 30, 23-30. [CrossRef]

177. Chen L.W.; Gu, J.Z. Correlations between the nuclear breathing mode energy and properties of asymmetric nuclear matter. J. Phys. G 2012, 39, 035104. [CrossRef]

178. Colo, G.; Garg, U.; Sagawa, H. Symmetry energy from the nuclear collective motion: Constraints from dipole, quadrupole, monopole and spin-dipole resonances. Eur. Phys. J. A 2014, 50, 26. [CrossRef]

179. Cai B.J.; Chen, L.W. Constraints on the skewness coefficient of symmetric nuclear matter within the nonlinear relativistic mean field model. Nucl. Sci. Tech. 2017, 28, 185. [CrossRef]

180. Han, S.; Prakash, M. On the minimum radius of very massive neutron stars. Astrophys. J. 2020, 899, 164. [CrossRef]

181. Somasundaram, R.; Margueron, J. Impact of massive neutron star radii on the nature of phase transitions in dense matter. arXiv 2021, arXiv:2104.13612. 